Supporting Information

\title{
Cu-Catalyzed Site-Selective Benzylic Chlorination Enabling Net C-H Coupling with Oxidatively Sensitive Nucleophiles
}

\author{
Marco Antonio Lopez, Joshua A. Buss, Shannon S. Stahl* \\ Department of Chemistry, University of Wisconsin-Madison, 1101 University Avenue \\ Madison, WI, 53706, United States \\ *stah1@chem.wisc.edu
}

Table of Contents:

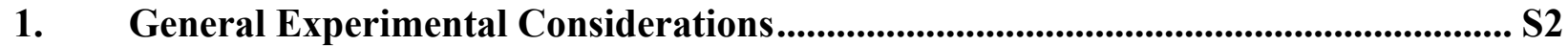

2. General Procedures for Benzylic C-H Chlorination Reactions ................................... S3

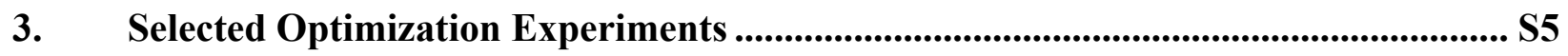

4. Procedures for Benzylic Chloride Displacement Reactions ....................................... S17

5. Product Synthesis and Characterization ..................................................................... S18

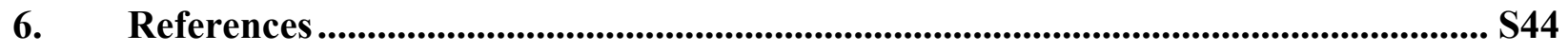

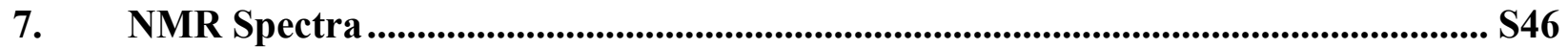




\section{General Experimental Considerations}

All reagents were purchased from commercial sources and used without any further purification. Benzylic C-H substrates were purchased from Aldrich, Alfa Aesar, Ark-Pharm, AstraTech, ChemImpex, Combi-Blocks, Enamine, Fischer-Scientific, TCI America, or Toronto Research Chemicals Inc, and were used without any further purification. ACS grade chlorobenzene was purchased from Aldrich, was dried over calcium hydride, then was degassed via three freeze-pump-thaw cycles prior to use. NFSI was purchased from Ark-Pharm and Oakwood. Crystalline potassium chloride was ground into a powder with a mortar and pestle. The purity of the phosphite reductant is critical to the success of the reaction and should be stored under inert atmosphere.

Ligand L4， (3aR,3a'R,8aS,8a'S)-2,2'-(cyclopropane-1,1-diyl)bis(3a,8a-dihydro-8H-indeno[1,2d]oxazole) was synthesized according to a literature procedure. ${ }^{1} \mathrm{CuCl}$ was prepared according to a literature procedure ${ }^{2}$ and stored in a $\mathrm{N}_{2}$ filled glovebox. (3,5-dibromo-4-methoxy-phenyl)-(2ethyl-benzofuran-3-yl)-methanone, precursor to $\mathbf{2 3}$, was synthesized according to the literature procedure. ${ }^{3}$ Trifluoroacetyl-protected amines, precursors to 12-14, were synthesized from the corresponding primary amines according to a literature procedure. ${ }^{4}$

Proton $\left({ }^{1} \mathrm{H}\right)$, carbon $\left({ }^{13} \mathrm{C}\right)$, and fluorine $\left({ }^{19} \mathrm{~F}\right)$ nuclear magnetic resonance (NMR) spectra were recorded on a Bruker $400 \mathrm{MHz}$ at $25{ }^{\circ} \mathrm{C}\left({ }^{1} \mathrm{H} 400.1 \mathrm{MHz},{ }^{13} \mathrm{C} 100.6 \mathrm{MHz},{ }^{19} \mathrm{~F} 376.5 \mathrm{MHz}\right)$, Bruker $500 \mathrm{MHz}$ at $25{ }^{\circ} \mathrm{C}\left({ }^{1} \mathrm{H} 500.1 \mathrm{MHz},{ }^{13} \mathrm{C} 125.7 \mathrm{MHz},{ }^{19} \mathrm{~F} 470.6 \mathrm{MHz}\right)$, or a $600 \mathrm{MHz}$ at $25^{\circ} \mathrm{C}\left({ }^{1} \mathrm{H}\right.$ $\left.600.1 \mathrm{MHz},{ }^{13} \mathrm{C} 151.1 \mathrm{MHz}\right)$. All spectrometers and chemical shifts are reported in parts per million (ppm). NMR spectra are referenced to residual solvent $\mathrm{CHCl}_{3}$ at $7.26 \mathrm{ppm}\left({ }^{1} \mathrm{H}\right)$ and $\mathrm{CDCl}_{3}$ at $77.16 \mathrm{ppm}\left({ }^{13} \mathrm{C}\right)$. All ${ }^{19} \mathrm{~F}$ NMR spectra were absolutely referenced to their respective solvent peaks in the ${ }^{1} \mathrm{H}$ NMR spectrum. High resolution mass spectrometry spectra were obtained on a Thermo Q Exactive ${ }^{\mathrm{TM}}$ Plus by the mass spectrometry facility at the University of WisconsinMadison. Automatic normal phase column chromatography was performed using reusable $25 \mathrm{~g}$ Sfär Silica HC D cartridges on a Biotage Isolera ${ }^{\circledR}$. Preparative thin-layer chromatography was performed using $20 \mathrm{~cm} \times 20 \mathrm{~cm}$ glass-backed silica preparative thin-layer chromatography plates (1000 um, layer thickness) from Miles Scientific. 


\section{General Procedures for Benzylic C-H Chlorination Reactions}

General Procedure: On the benchtop, a disposable vial was charged with benzylic substrate (1 equiv), N-Fluorobenzenesulfonimide (NFSI; 2.5 equiv), potassium chloride ( $\mathrm{KCl} ; 3$ equiv), and a Teflon stir bar. The vial was sealed by a PTFE-lined pierceable cap. Bis(oxazoline) ligand L4 was weighed into a secondary vial with a Teflon stir bar*. Both vials were sparged with $\mathrm{N}_{2}$ then transferred into a purging glovebox under $\mathrm{N}_{2}$. In the glovebox, $\mathrm{CuCl}$ was weighed into the vial containing Bis(oxazoline) ligand. Chlorobenzene was added to this vial and the vial was stirred for $15 \mathrm{~min}$ to form a clear light yellow stock solution of copper catalyst. To the vial containing benzylic substrate, NFSI, KCl, and the Teflon stir bar was added diisopropyl phosphite (1 equiv) and the copper catalyst stock solution to form a green solution. The reaction vial was then sealed, removed from the glovebox, and set to stir at $750 \mathrm{rpm}$ on an aluminum block at the specified temperature for $16 \mathrm{~h}$.

Work Up: After $16 \mathrm{~h}$, mesitylene $(30 \mu \mathrm{L}, 0.215 \mathrm{mmol})$ or dibromethane $\mathrm{CH}_{2} \mathrm{Br}_{2}(0.427 \mathrm{mmol}, 30$ $\mu \mathrm{L} 2.36$ equiv) was added to the crude heterogenous reaction mixture as an external NMR standard. The crude reaction mixture was filtered through a pad of silica using $\mathrm{CHCl}_{3}$. An aliquot $(30 \mu \mathrm{L})$ from the now homogenous solution was withdrawn for NMR analysis to detect formation of product and consumption of starting material. After NMR analysis, the crude reaction mixture was concentrated on the rotovap at $30^{\circ} \mathrm{C}$ to remove solvent (chlorobenzene and chloroform). The obtained residue was purified by flash column chromatography.

\section{Procedure A (0.2 mmol).}

Vial 1: a disposable 1 dram vial, benzylic substrate (0.2 mmol, 1 equiv), NFSI (157.7 $\mathrm{mg}, 0.5$ mmol, 2.5 equiv), $\mathrm{KCl}$ (44.7 mg, $0.6 \mathrm{mmol}, 3$ equiv), and a Teflon stir bar* .

Vial 2: Bis(oxazoline) ligand (L2, 21.4. $\mathrm{mg}, 0.06 \mathrm{mmol}), \mathrm{CuCl}(6.0 \mathrm{mg}, 0.06 \mathrm{mmol})$, and chlorobenzene $(3 \mathrm{~mL})$ combined to form a clear light yellow $0.02 \mathrm{M}$ stock solution of copper catalyst.

To Vial 1 was added diisopropyl phosphite $(33 \mu \mathrm{L}, 0.2 \mathrm{mmol}, 1$ equiv) and $1 \mathrm{~mL}$ of the copper catalyst stock solution. The reaction was conducted at room temperature.

Work Up: Mesitylene ( $30 \mu \mathrm{L}, 0.215 \mathrm{mmol}$ ) was added to the crude heterogenous reaction mixture and worked up as outlined in the General Procedure.

Procedure B (0.2 mmol). Adopting Procedure A, the reaction was performed at $35{ }^{\circ} \mathrm{C}$.

Procedure C (0.2 mmol). Adopting Procedure A, the reaction was performed at $45^{\circ} \mathrm{C}$.

Procedure D (0.2 mmol) Adopting Procedure A, the reaction was performed at $70{ }^{\circ} \mathrm{C}$.

\section{Procedure E (0.5 mmol).}

Vial 1: a disposable $24 \mathrm{~mL}$ glass vial, benzylic substrate $(0.5 \mathrm{mmol}, 1$ equiv), NFSI (394.2 $\mathrm{mg}$, $1.25 \mathrm{mmol}, 2.5$ equiv), $\mathrm{KCl}$ (111.8 mg, $1.5 \mathrm{mmol}, 3$ equiv), and two Teflon stir bars (see note on stir bars below).

Vial 2: Bis(oxazoline) ligand (L2, $53.5 \mathrm{mg}, 0.15 \mathrm{mmol}), \mathrm{CuCl}$ (14.8 $\mathrm{mg}, 0.15 \mathrm{mmol}$ ), and chlorobenzene $(7.5 \mathrm{~mL})$ combined to form a clear light yellow $0.02 \mathrm{M}$ stock solution of copper catalyst.

To Vial 1 was added diisopropyl phosphite ( $83 \mu \mathrm{L}, 0.5 \mathrm{mmol}, 1$ equiv) and $2.5 \mathrm{~mL}$ of the copper catalyst stock solution. The reaction was conducted at room temperature. 
Work Up: Mesitylene ( $30 \mu \mathrm{L}, 0.215 \mathrm{mmol})$ was added to the heterogenous solution and worked up as outlined in the General Procedure.

Procedure F (0.5 mmol). Adopting Procedure D, the reaction was performed at $35{ }^{\circ} \mathrm{C}$.

Procedure G $(0.5 \mathrm{mmol})$. Adopting Procedure D, the reaction was performed at $45^{\circ} \mathrm{C}$.

\section{Procedure H (1.2 mmol; used for products 13 and 24).}

Vial 1: a disposable $24 \mathrm{~mL}$ glass vial benzylic substrate (1.2 mmol, 1 equiv), NFSI (946.0 mg, 3 mmol, 2.5 equiv), $\mathrm{KCl}$ (268.4 mg, $3.6 \mathrm{mmol}, 3$ equiv), and two Teflon stir bars.

Vial 2: Bis(oxazoline) ligand (L2, $42.77 \mathrm{mg}, 0.12 \mathrm{mmol}, 0.1$ equiv), $\mathrm{CuCl}$ (11.99 mg, $0.12 \mathrm{mmol}$, 0.1 equiv), and chlorobenzene $(5 \mathrm{~mL})$ to form a clear light yellow $0.24 \mathrm{M}$ stock solution of copper catalyst.

To Vial 1 was added diisopropyl phosphite ( $199 \mu \mathrm{L}, 1.2 \mathrm{mmol}, 1$ equiv), and $5 \mathrm{~mL}$ of the copper catalyst stock solution. The reaction was conducted at $35^{\circ} \mathrm{C}$.

Work up: After $16 \mathrm{~h}$, mesitylene $(30 \mu \mathrm{L}, 0.215 \mathrm{mmol})$ was added to the heterogenous solution and worked up as outlined in the General Procedure.<smiles>O=C(NCCC(Cl)c1ccccc1)C(F)(F)F</smiles>

13

Amount Isolated: $229 \mathrm{mg}$ Percent Yield: $72 \%$<smiles>CCCC(Cl)c1oc2ccc([N+](=O)[O-])cc2c1C(=O)c1ccc(OC)cc1</smiles>

Amount Isolated: $414 \mathrm{mg}$ Percent Yield: 89\%

\section{Notes:}

- Vigorous stirring is crucial to the success of the reaction.

- The position of vial on the aluminum heating block can affect the yields of the desired benzylic chloride. Higher yields are observed when the reaction vial is closer to the center of the aluminum block.

- The reaction solvent in the reaction vessel should be completely below the level of the heating block.

- Evaporation of solvent for compounds on the rotary evaporator should be carefully conducted at low temperature due to their high volatility

- Stir bar size is critical to the success of the chlorination reactions. All reactions were carried out in in either 1 dram or $24 \mathrm{~mL}$ reaction vials using one or two $3 \mathrm{~mm}$ x $12 \mathrm{~mm}$ stir bars (Figure S1).
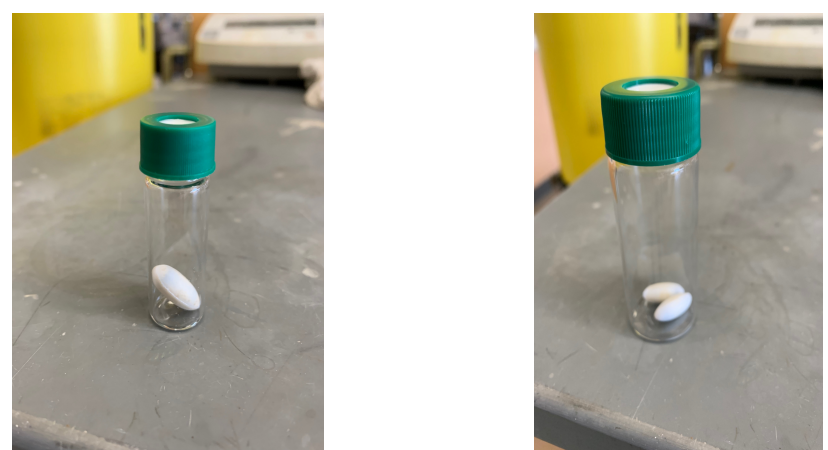

Figure S1. A 1 dram vial for $0.2 \mathrm{mmol}$ reaction with small stir bar (left), and a $24 \mathrm{~mL}$ vial for 0.5 and $1.2 \mathrm{mmol}$ reaction with two stir bars (right). 


\section{Selected Optimization Experiments}

\section{General Procedure for Optimization of Reaction Parameters.}

Set up: On the benchtop, a disposable 1 dram vial was charged with isobutyl benzene (1a) (0.2 mmol, 1 equiv), NFSI, chloride source, Cu salt, ligand, reductant, and a Teflon stir bar. The vial was sealed by a PTFE-lined pierceable cap and then transferred into a purging glovebox under $\mathrm{N}_{2}$. In the glovebox, chlorobenzene $(1 \mathrm{~mL})$ was added to this vial to form a green solution. The reaction vial was then sealed, removed from the glovebox, and stirred at $750 \mathrm{rpm}$ on an aluminum block at $45^{\circ} \mathrm{C}$ for $16 \mathrm{~h}$.

Work Up: After $16 \mathrm{~h}$, mesitylene $(30 \mu \mathrm{L}, 0.215 \mathrm{mmol})$ was added to the crude heterogenous reaction mixture as an external NMR standard. The crude reaction mixture was filtered through a pad of silica directly into a test tube using $\mathrm{CHCl}_{3}$. An aliquot $(30 \mu \mathrm{L})$ from the now homogenous solution was taken for NMR analysis to detect formation of product and consumption of starting material.

Table S1. Control Experiments.

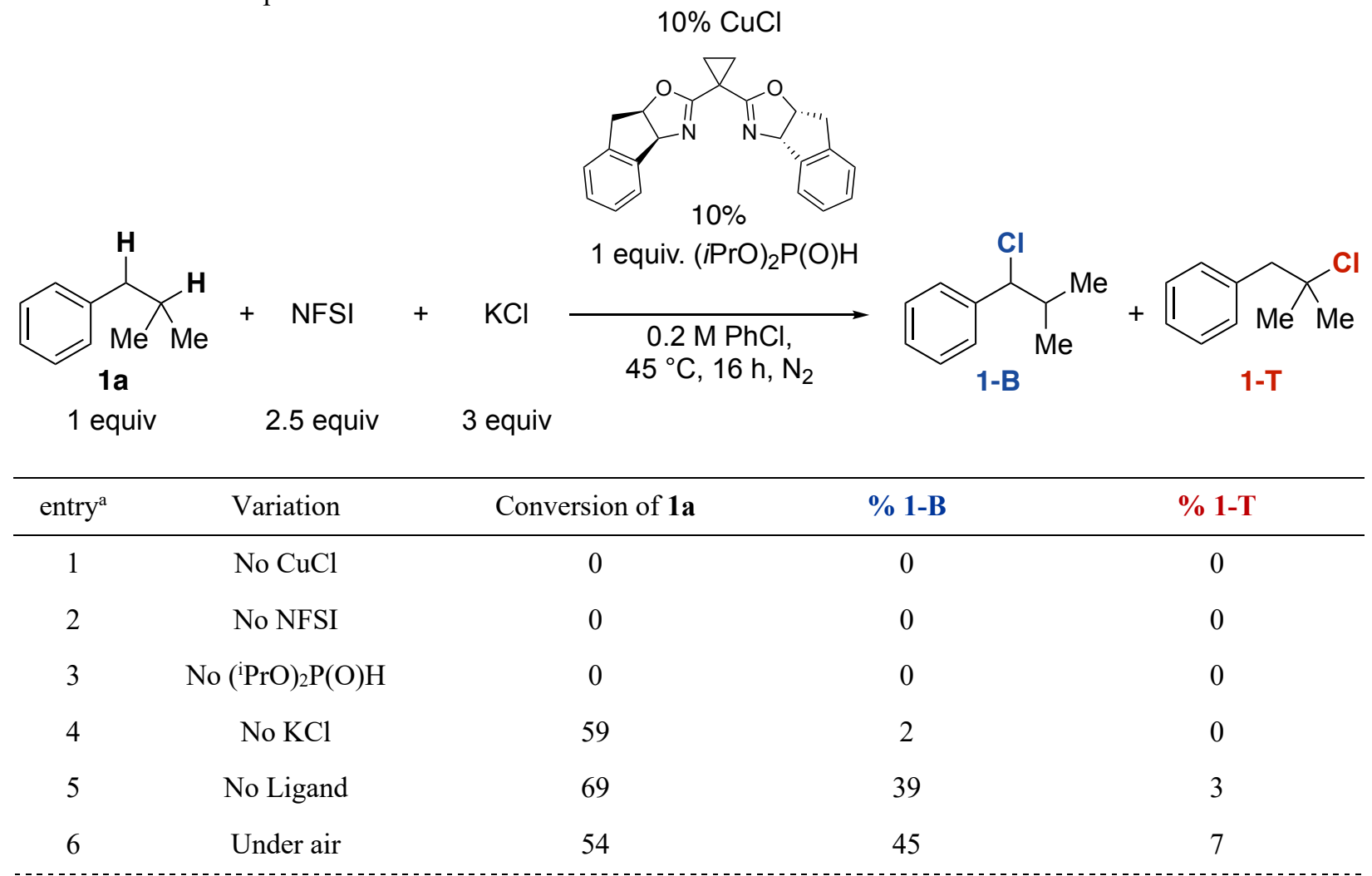

${ }^{a}$ Reactions were run on a 0.2 mmol scale. Calibrated ${ }^{1} \mathrm{H}$ NMR yields were determined versus mesitylene as the external standard. 
Table S2. Evaluation of Solvent.

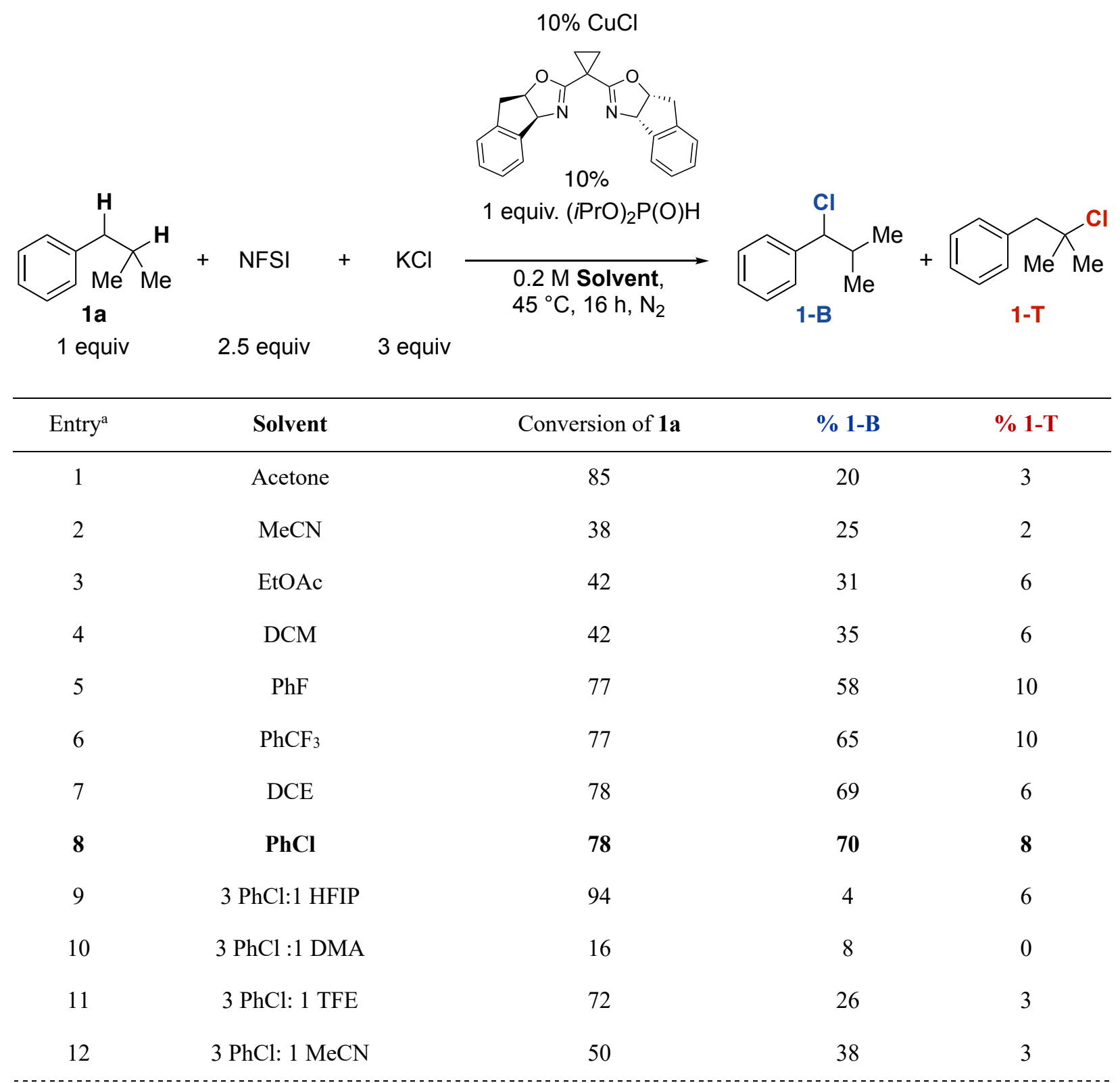

${ }^{a}$ Reactions were run on a $0.2 \mathrm{mmol}$ scale. Calibrated ${ }^{\mathrm{H}} \mathrm{H}$ NMR yields were determined versus mesitylene as the external standard. DCM, Dichloromethane; DCE, 1,2-dichloroethane; ACN, acetonitrile; EtOAc, ethyl acetate; PhF, Fluorobenzene; $\mathrm{PhCF}_{3}$, Trifluorotoluene; $\mathrm{PhCl}$, Chlorobenzene; HFIP, Hexafluoroisopropanol; TFE, Trifluoroethanol; DMA, N,N-dimethylacetamide.

Discussion: The results reported in Table S2 shows the chlorination reaction outcome when employing different solvents. Chlorobenzene was selected as the optimal solvent for this transformation as it afforded the highest yields and selectivity for $2^{\circ}$ benzylic chlorination. However, trifluorotoluene and dichloroethane also proved to be suitable solvents for this transformation and can be used as alternatives. 
Table S3. Evaluation of Ancillary Ligands.

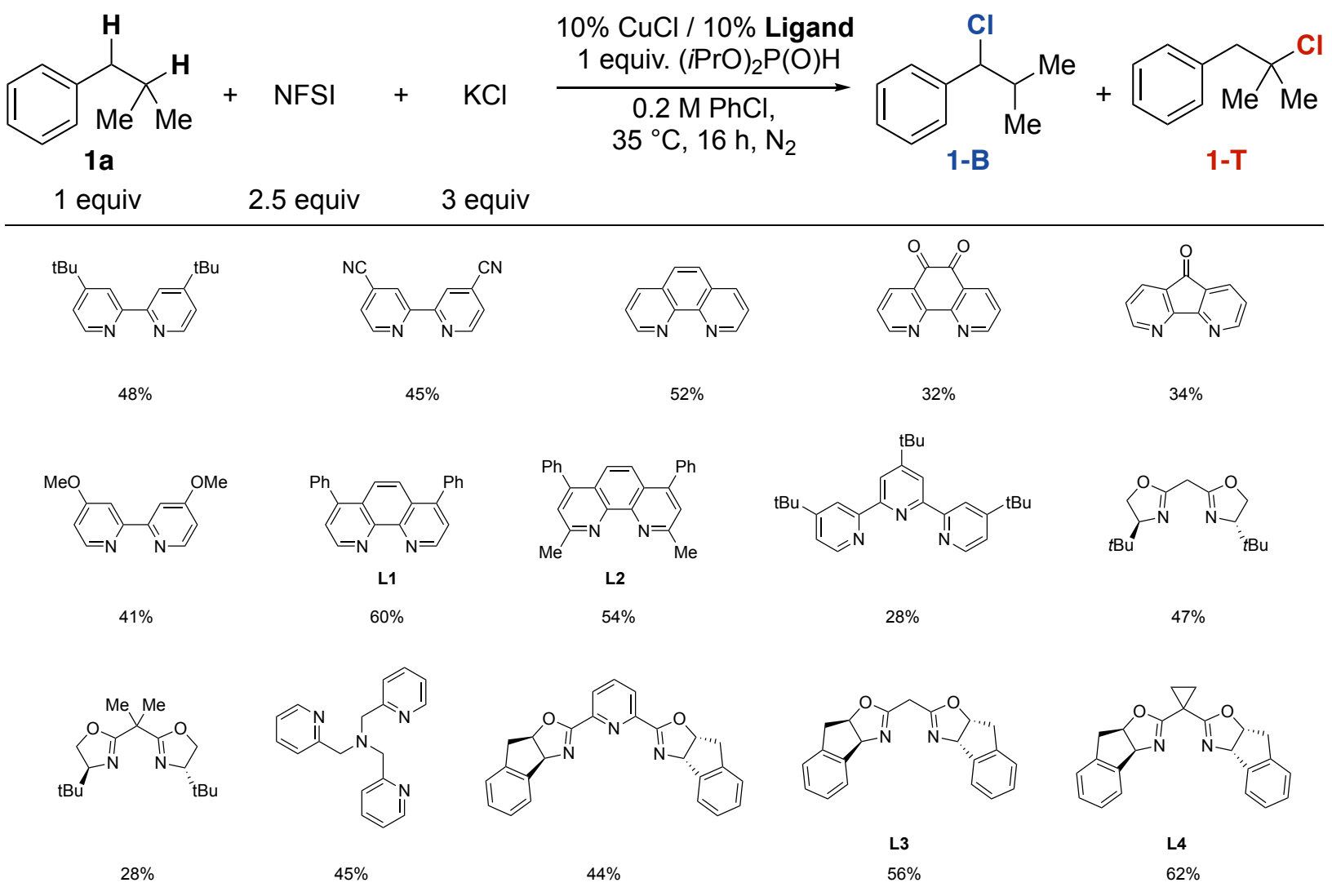

${ }^{\mathrm{a}}$ Reactions were run on a $0.2 \mathrm{mmol}$ scale. Calibrated ${ }^{1} \mathrm{H}$ NMR yields were determined versus mesitylene as the external standard.

Discussion: The results reported in Table $\mathbf{S 3}$ shows the chlorination reaction outcome with various ancillary ligands. While bathophenanthroline (L1) and bathocuproine (L2) demonstrated to be effective ligands for the chlorination reaction on isobutylbenzene, Bis(oxazoline) ligand (L4) proved to be slightly more effective in this screen. Evaluation of L1, L2, and L4 (see Figure S2) on different substrates demonstrates improved reactivity with $\mathbf{L} \mathbf{4}$ 
Table S4. Evaluation of Reductants
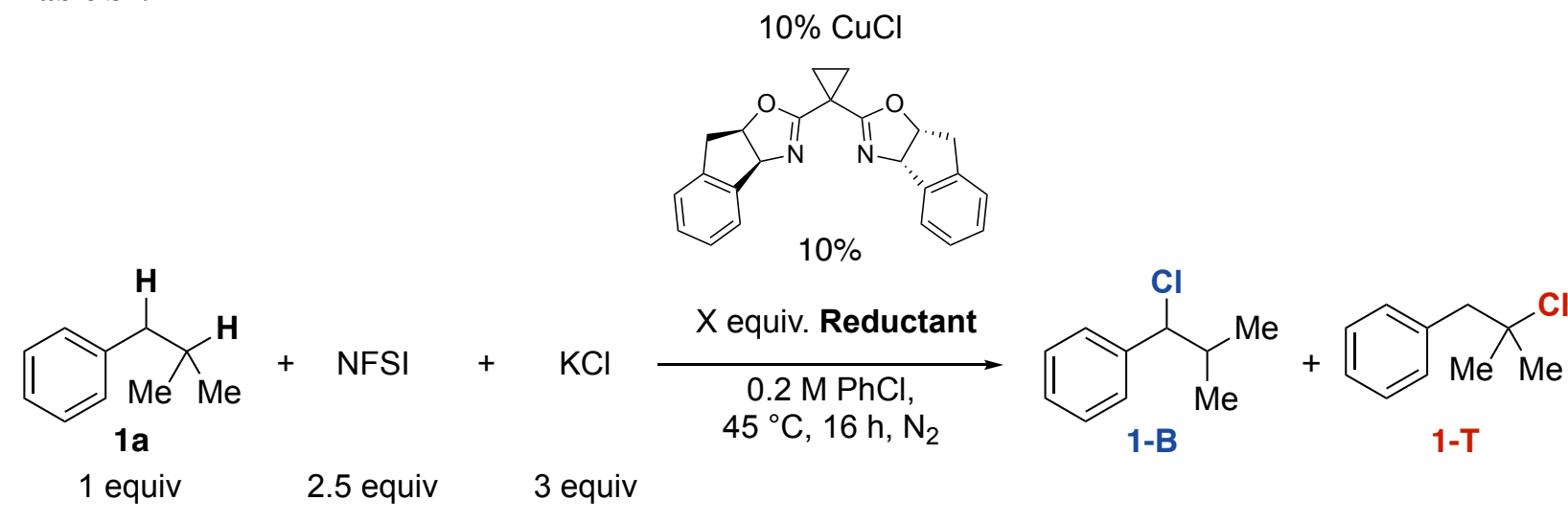

\begin{tabular}{ccccc}
\hline Entry $^{\mathrm{a}}$ & Reductant & Conversion of 1a & \%Cl 1-B & \% Cl 1-T \\
\hline 1 & 0.5 equiv $(\mathrm{iPrO})_{2} \mathrm{P}(\mathrm{O}) \mathrm{H}$ & 85 & 59 & 6 \\
$\mathbf{2}$ & $\mathbf{1}$ equiv (iPrO) $)_{2} \mathrm{P}(\mathbf{O}) \mathbf{H}$ & $\mathbf{8 5}$ & $\mathbf{6 3}$ & $\mathbf{5}$ \\
3 & 1.5 equiv $(\mathrm{iPrO})_{2} \mathrm{P}(\mathrm{O}) \mathrm{H}$ & 73 & 57 & 4 \\
4 & 2.0 equiv $(\mathrm{iPrO})_{2} \mathrm{P}(\mathrm{O}) \mathrm{H}$ & 69 & 59 & 5 \\
5 & 1 equiv $(\mathrm{PhO})_{2} \mathrm{P}(\mathrm{O}) \mathrm{H}$ & 0 & 0 & 0 \\
6 & 1 equiv $(\mathrm{MeO})_{2} \mathrm{P}(\mathrm{O}) \mathrm{H}$ & 72 & 52 & 6 \\
7 & 1 equiv $(\mathrm{EtO})_{2} \mathrm{P}(\mathrm{O}) \mathrm{H}$ & 65 & 55 & 8 \\
8 & 1 equiv $(\mathrm{tBuO})_{2} \mathrm{P}(\mathrm{O}) \mathrm{H}$ & 35 & 28 & 3 \\
9 & 1 equiv $(\mathrm{MeO})_{2} \mathrm{MeSiH}$ & 25 & 20 & 2
\end{tabular}

${ }^{a}$ Reactions were run on a $0.2 \mathrm{mmol}$ scale. Calibrated ${ }^{1} \mathrm{H}$ NMR yields were determined versus mesitylene as the external standard.

Discussion: The results reported in Table $\mathbf{S} 4$ shows the chlorination reaction outcome with various silane and phosphite based reductants. Diisopropyl phosphite (1 equiv) proved to be the most effective for this transformation. 
Table S5. Evaluation of $\mathrm{Cu}$ Salts
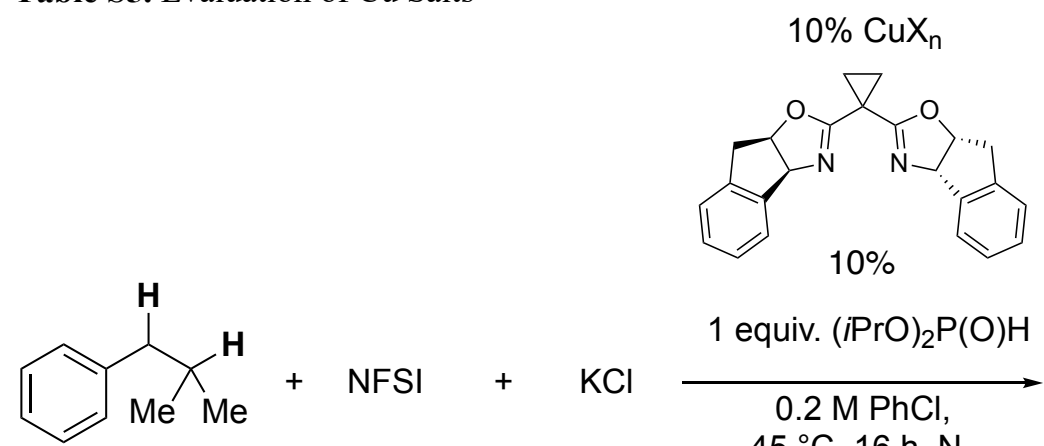

1 a
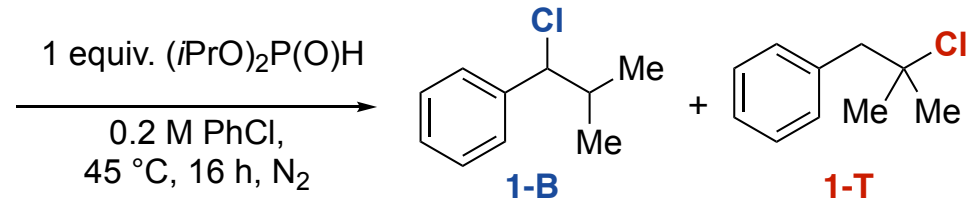

1 equiv

2.5 equiv

3 equiv

\begin{tabular}{|c|c|c|c|c|}
\hline Entry $^{\mathrm{a}}$ & Cu Salt & Conversion of $\mathbf{1 a}$ & $\%$ Cl 1-B & $\%$ Cl 1-T \\
\hline 1 & CuOTf•Toluene & 45 & 40 & 3 \\
\hline 2 & $\mathrm{Cu}(\mathrm{OTf})_{2}$ & 47 & 43 & 3 \\
\hline 3 & $\mathrm{CuOAc}$ & 65 & 52 & 6 \\
\hline 4 & $\mathrm{Cu}(\mathrm{OAc})_{2}$ & 60 & 54 & 8 \\
\hline 5 & $\mathrm{Cu}(\mathrm{MeCN})_{4} \mathrm{PHF}_{3}$ & 69 & 56 & 7 \\
\hline 6 & $\mathrm{CuBr} \bullet \mathrm{DMS}$ & 65 & 58 & 5 \\
\hline 7 & $\mathrm{CuI} \cdot \mathrm{DMS}$ & 66 & 60 & 7 \\
\hline 8 & $\mathrm{CuCl}_{2}$ & 68 & 60 & 5 \\
\hline 9 & $\mathrm{CuCN}$ & 72 & 63 & 9 \\
\hline 10 & $\mathrm{CuCl}$ & 78 & 69 & 6 \\
\hline
\end{tabular}

The results reported in Table $\mathbf{S 5}$ shows the chlorination reaction outcome with various $\mathrm{Cu}$ salts. 
Table S6. Evaluation of $\mathrm{Cu} /$ Ligand Loading.

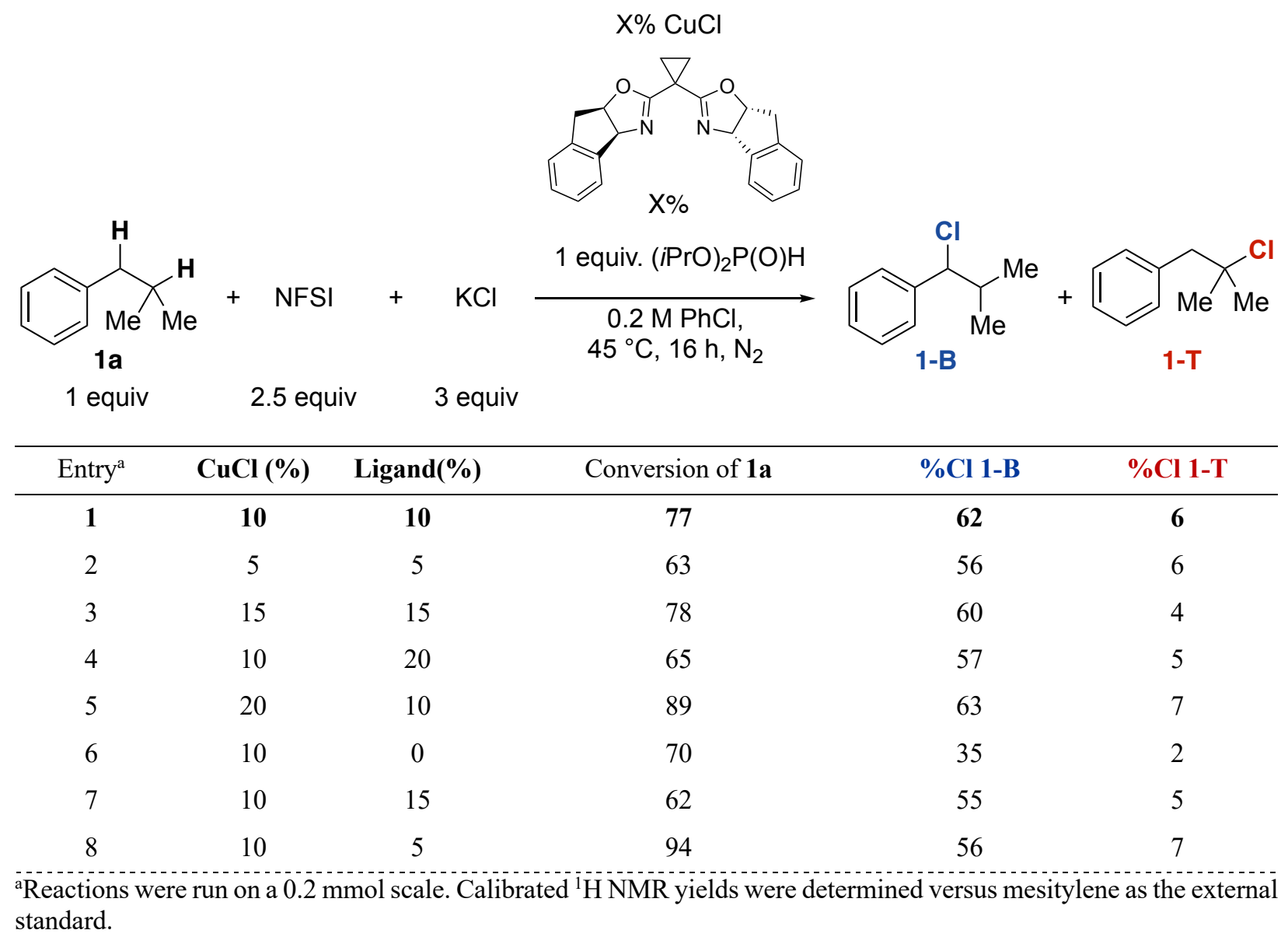


Table S7. Evaluation of NFSI Loading

\begin{tabular}{|c|c|c|c|c|c|}
\hline$\underbrace{1 \text { equiv }}_{1 \text { Me }}$ & $\begin{array}{c}+\quad \mathrm{NFSI} \\
\times \text { equiv }\end{array}$ & $\begin{array}{c}\mathrm{KCl} \\
3 \text { equiv }\end{array}$ & $\begin{array}{c}1 \text { equiv. (iPrO) }{ }_{2} \mathrm{P}(\mathrm{O} \\
0.2 \mathrm{M} \mathrm{PhCl} \\
45^{\circ} \mathrm{C}, 16 \mathrm{~h}, \mathrm{~N}_{2}\end{array}$ & $\underbrace{C I}_{M e}$ & $\int_{1-T}{ }_{1-T}$ \\
\hline Entry $^{a}$ & NFSI Equiv & & Conversion of $\mathbf{1 a}$ & $\% \mathrm{Cl} 1-\mathrm{B}$ & $\% \mathrm{Cl} 1-\mathrm{T}$ \\
\hline 1 & 1 & & 35 & 30 & 3 \\
\hline 2 & 1.5 & & 52 & 46 & 5 \\
\hline 3 & 2 & & 76 & 67 & 7 \\
\hline 4 & 2.5 & & 80 & 69 & 7 \\
\hline 5 & 3 & & 91 & 68 & 6 \\
\hline 6 & 3.5 & & 88 & 60 & 8 \\
\hline 7 & 4 & & 99 & 59 & 6 \\
\hline 8 & 5 & & 89 & 63 & 8 \\
\hline
\end{tabular}


Table S8. Evaluation of Chloride Sources.

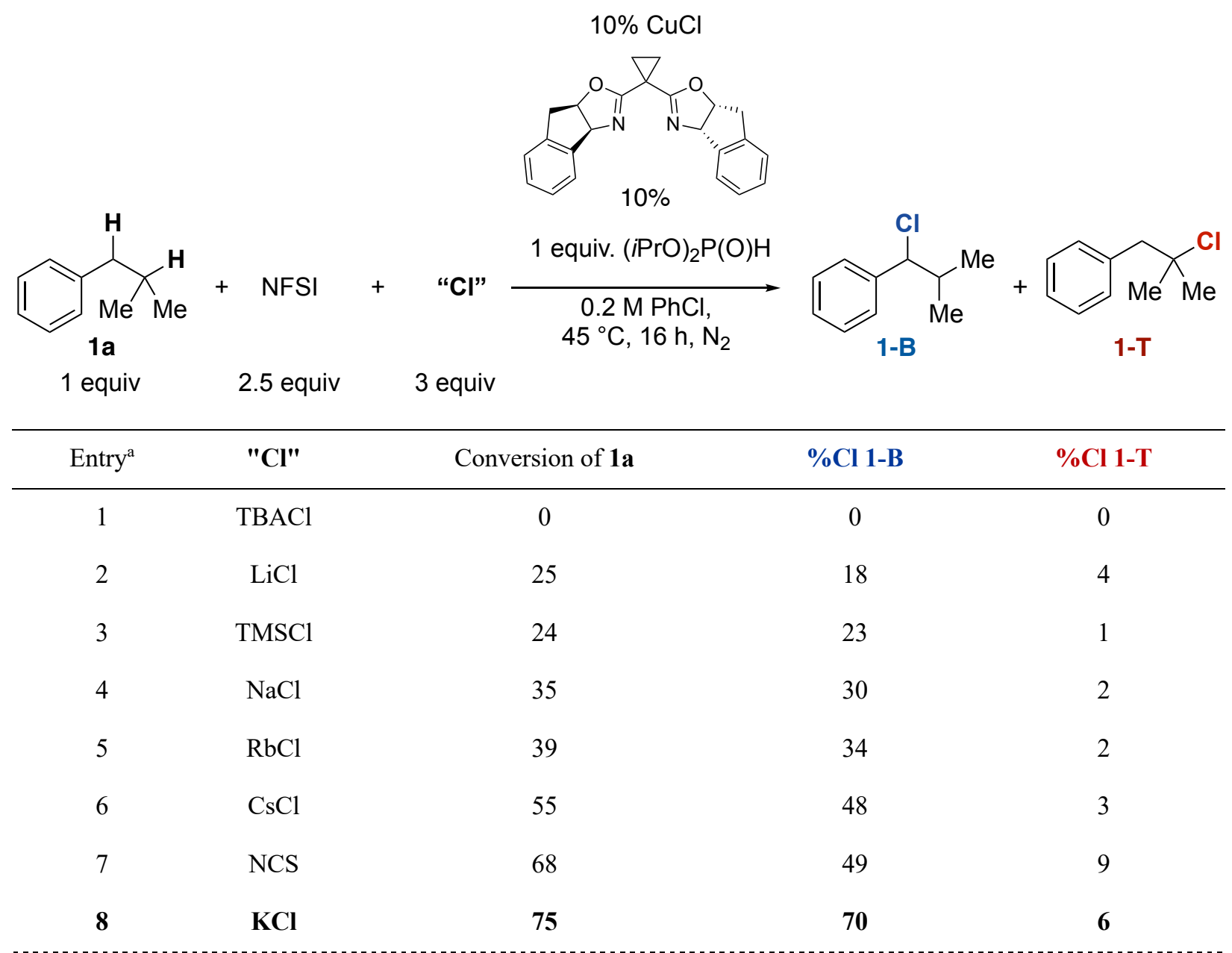

${ }^{a}$ Reactions were run on a $0.2 \mathrm{mmol}$ scale. Calibrated ${ }^{1} \mathrm{H}$ NMR yields were determined versus mesitylene as the external standard. TBAC, Tetrabutylammonium chloride; TMSCl, Trimethylsilyl chloride, NCS, N-Chlorosuccinimide 


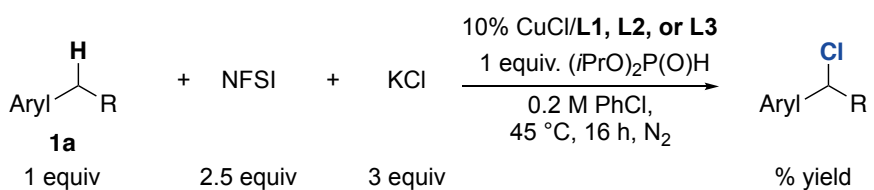

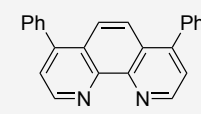

L1

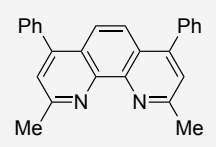

L2

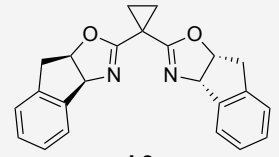

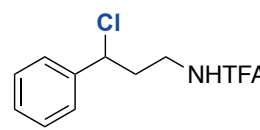

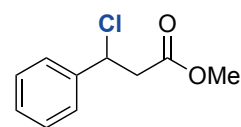

L1, 33\%

L2, $54 \%$

L3, $74 \%$

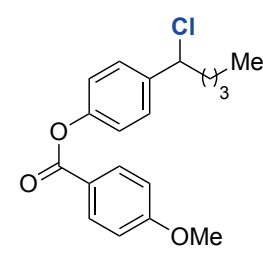

L1, $72 \%$
L2, $87 \%$

L3, $89 \%$

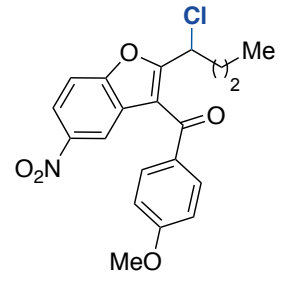

L1, $69 \%$

L2, $85 \%$

L3, $82 \%$

Figure S2. Comparative study of $\mathbf{L 1}, \mathbf{L 2}$, and $\mathbf{L} 4$ with various $\mathrm{C}-\mathrm{H}$ substrate. ${ }^{\mathrm{a}}$ Reactions were run on a $0.2 \mathrm{mmol}$ scale. Calibrated ${ }^{1} \mathrm{H}$ NMR yields were determined versus mesitylene as the external standard. 
Table S9. Comparative Data with Known Chlorination Methods ${ }^{a}$

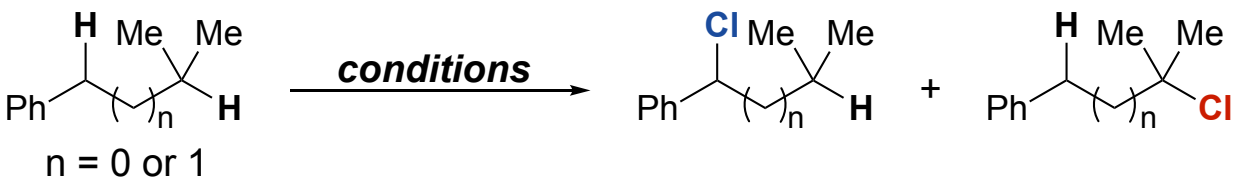

\begin{tabular}{|c|c|c|c|c|}
\hline Conditions $a-h$ & $\begin{array}{c}\% \mathrm{Cl}-1-\mathrm{B} \\
\mathrm{n}=0\end{array}$ & $\begin{array}{c}\% \mathrm{Cl}-1-\mathrm{T} \\
\mathrm{n}=0\end{array}$ & $\begin{array}{c}\% \mathrm{Cl} 2-\mathrm{B} \\
\mathrm{n}=1\end{array}$ & $\begin{array}{c}\% \mathrm{Cl} 2-\mathrm{T} \\
\mathrm{n}=2\end{array}$ \\
\hline $\begin{array}{c}a . \\
10 \% \mathrm{CuCl} / \mathrm{L}, 2.5 \mathrm{NFSI}, 3 \mathrm{KCl} \text {, }\end{array}$ & 69 & 7 & 78 & 4 \\
\hline $\begin{array}{c}\boldsymbol{b}^{5} \\
\mathrm{AgOTf} / \text { phen, } \mathrm{tBuOCl}\end{array}$ & 37 & 21 & 53 & 29 \\
\hline $\begin{array}{c}c .^{6} \\
\text { NCS, DPBO, cat. HOAc }\end{array}$ & 30 & 17 & 39 & 12 \\
\hline $\begin{array}{c}\boldsymbol{d}^{7} \\
\mathrm{Cu}(\mathrm{OAc})_{2} 40 \% \mathrm{TCCA}, 10 \% \text { NHPI }\end{array}$ & 25 & 9 & 27 & 13 \\
\hline $\begin{array}{c}\boldsymbol{e}^{8} \\
5 \% \mathrm{Ph}_{2} \mathrm{CO}, \mathrm{NCS}, h v\end{array}$ & 18 & 13 & 10 & 8 \\
\hline $\begin{array}{c}\boldsymbol{f}^{9} \\
\mathrm{SO}_{2} \mathrm{Cl}_{2} \text {, cat. } \mathrm{DBPO}, 45^{\circ} \mathrm{C}\end{array}$ & 17 & 14 & 18 & 26 \\
\hline $\begin{array}{l}\text { g. }{ }^{9} \\
\mathrm{SO}_{2} \mathrm{Cl}_{2}, \text { cat. } \mathrm{DBPO}, 75^{\circ} \mathrm{C}\end{array}$ & 28 & 31 & 20 & 23 \\
\hline $\begin{array}{c}\boldsymbol{h}^{10}{ }^{2} \\
2 \% \mathrm{Mn}(\mathrm{TPP}) \mathrm{Cl}, \mathrm{NaOCl}, 4 \% \mathrm{TBACl}\end{array}$ & 9 & 6 & 9 & 3 \\
\hline
\end{tabular}

${ }^{a}$ Site-selectivity studies were performed on a $0.2 \mathrm{mmol}$ scale according to the literature procedures. The site-selectivity was determined by ${ }^{1} \mathrm{H}$ NMR spectroscopy using mesitylene $(0.215 \mathrm{mmol}, 30 \mu \mathrm{L}, 1.07$ equiv $)$ as the external standard. 


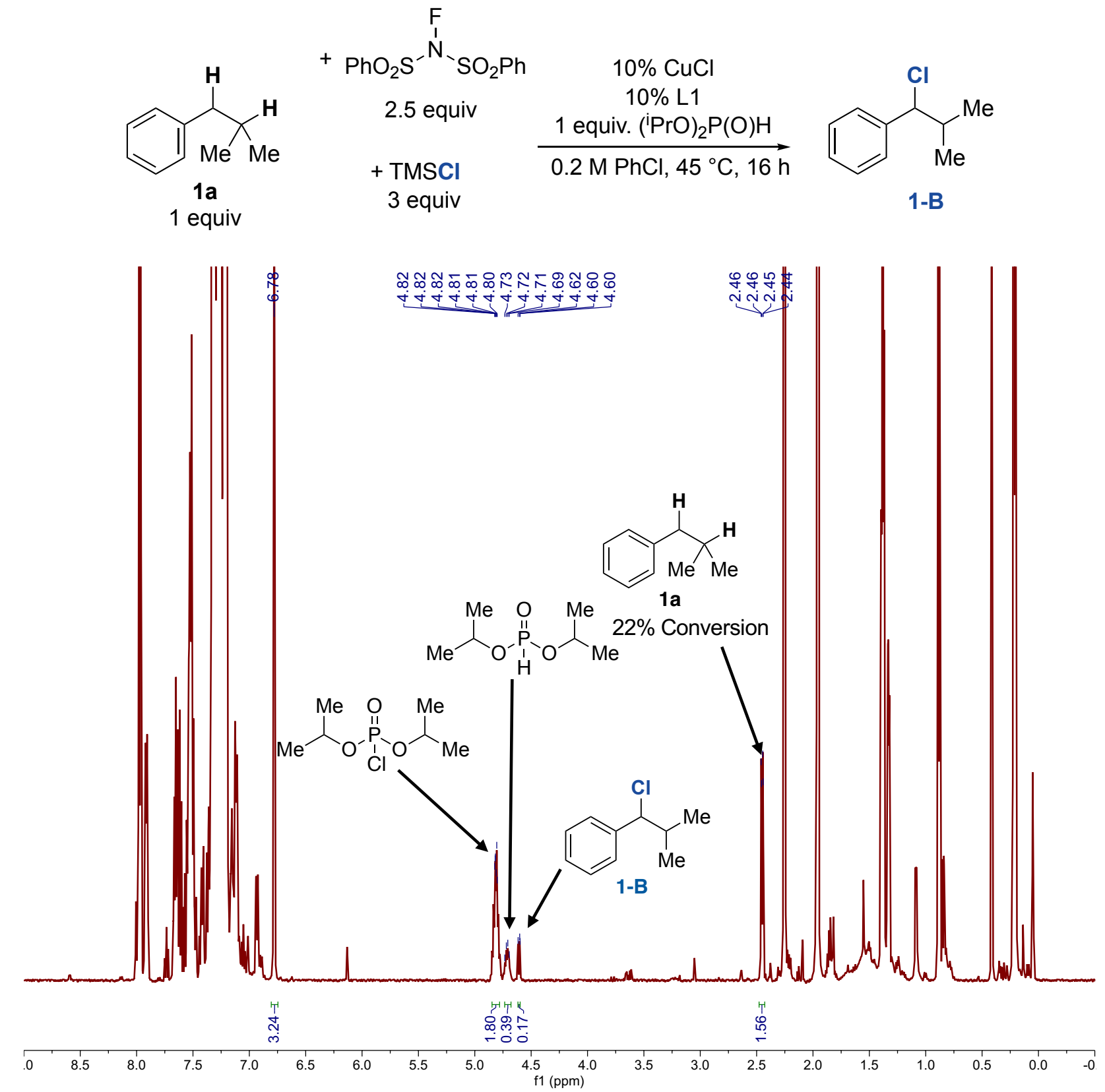

Figure S3. Crude ${ }^{1} \mathrm{H}$ NMR Spectrum $\left(\mathrm{CDCl}_{3}, 400 \mathrm{MHz}, 25^{\circ} \mathrm{C}\right)$ of the reaction mixture for 1-B following the addition of $0.215 \mathrm{mmol}(30 \mu \mathrm{L})$ of mesitylene as an external standard $(6.78 \mathrm{ppm})$. The resolved methine protons for diisopropyl chlorophosphate $^{11}(4.83 \mathrm{ppm}, \mathrm{m})$ and diisopropyl phosphite ${ }^{12}(4.71 \mathrm{ppm}, \mathrm{m})$ are labeled and integrated. The resolved benzylic protons for both 1-B $(4.62 \mathrm{ppm}, \mathrm{d})$ and $1 \mathrm{a}(2.45 \mathrm{ppm}, \mathrm{d})$ chlorides are labeled and integrated. ${ }^{\text {Reactions }}$ were run on a $0.2 \mathrm{mmol}$ scale. Calibrated ${ }^{1} \mathrm{H}$ NMR yields were determined versus mesitylene as the external standard. 


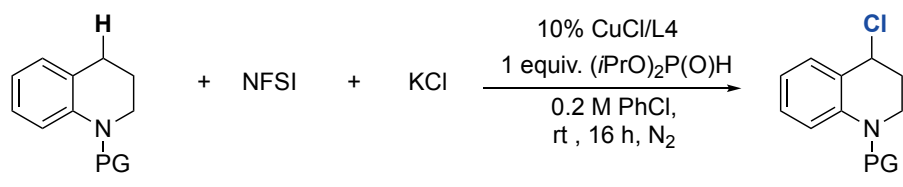

1 equiv 2.5 equiv 3 equiv $\quad \%$ yield

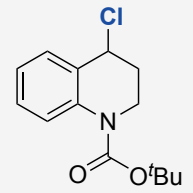

Con $100 \%$

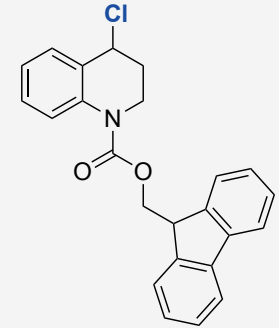

Con $100 \%$

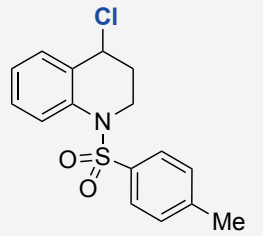

Con $84 \%$

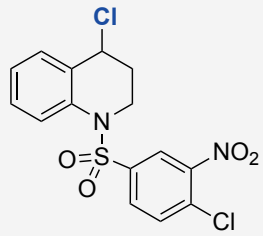

Con $84 \%$

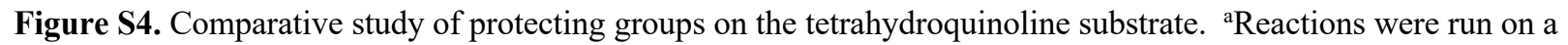
$0.2 \mathrm{mmol}$ scale. Calibrated ${ }^{1} \mathrm{H}$ NMR yields were determined versus mesitylene as the external standard. 


\section{Procedures for Benzylic Chloride Displacement Reactions}

General Procedure I for Chloride Displacement with Phenols and Thiophenols: In the fume hood, a disposable 1 dram vial was charged with (thio)phenol ( $0.4 \mathrm{mmol}, 2$ equiv), potassium iodide (KI; $0.21 \mathrm{mmol}, 34.9 \mathrm{mg}, 1.05$ equiv), potassium carbonate $\left(\mathrm{K}_{2} \mathrm{CO}_{3} ; 0.41 \mathrm{mmol}, 56.7 \mathrm{mg}\right.$, 2 equiv) and a Teflon stir bar. The vial was subsequently charged with $2 \mathrm{~mL}$ of a $0.1 \mathrm{M}$ benzylic chloride stock solution in DMF. The vial was sealed by a PTFE-lined pierceable cap and set to stir at on an aluminum block at $70{ }^{\circ} \mathrm{C}$ and stirred at $750 \mathrm{rpm}$ for $16 \mathrm{~h}$.

Work Up: After $16 \mathrm{~h}$, mesitylene $(0.215 \mathrm{mmol}, 30 \mu \mathrm{L})$ was added to the crude heterogenous reaction mixture as the external NMR standard. The crude reaction mixture was filtered through a pad of silica directly into a $24 \mathrm{~mL}$ vial using dichloromethane $(5 \mathrm{~mL})$. An aliquot $(30 \mu \mathrm{L})$ from the now homogenous solution was taken for NMR analysis to detect formation of product and consumption of starting material. After NMR analysis, crude reaction solution was washed with a $1.0 \mathrm{M} \mathrm{NaOH}$ solution (3x). The organic layer was dried with sodium sulfate, filtered through a pad of celite, and concentrated under reduced pressure. The resulting residue was purified by flash column chromatography.

General Procedure $\mathbf{J}$ for Benzylic Chloride Displacement with Amines: In the fume hood, a disposable 1 dram vial was charged with amine $(0.4 \mathrm{mmol}, 2$ equiv), potassium iodide (KI; 0.21 mmol, $34.9 \mathrm{mg}, 1.05$ equiv), and a Teflon stir bar. The vial was subsequently charged with $2 \mathrm{~mL}$ of a $0.1 \mathrm{M}$ benzylic chloride stock solution in $\mathrm{MeCN}$. The vial was sealed by a PTFE-lined pierceable cap and set to stir at on an aluminum block at $70{ }^{\circ} \mathrm{C}$ and stirred at $700 \mathrm{rpm}$ for $16 \mathrm{~h}$.

Work Up: After $16 \mathrm{~h}$, mesitylene $(0.215 \mathrm{mmol}, 30 \mu \mathrm{L})$ was added to the crude heterogenous reaction mixture as the external NMR standard. The crude reaction mixture was filtered through a pad of silica directly into a $24 \mathrm{~mL}$ vial using dichloromethane $(5 \mathrm{~mL})$. An aliquot $(30 \mu \mathrm{L})$ from the now homogenous solution was taken for NMR analysis to detect formation of product and consumption of starting material. After NMR analysis, crude reaction solution was washed with a $1.0 \mathrm{M} \mathrm{NaOH}$ solution (3x). The organic layer was dried with sodium sulfate, filtered through a pad of celite, and concentrated under reduced pressure. The resulting residue was purified by flash column chromatography. 


\section{Product Synthesis and Characterization}

\section{(1-chloro-2-methylpropyl)benzene (1-B)}<smiles>CC(C)C(Cl)c1ccccc1</smiles>

Reaction conducted using Procedure G with isobutylbenzene $(67.1 \mathrm{mg}, 78.6 \mu \mathrm{L}, 0.5 \mathrm{mmol}, 1$ equiv). Following workup, crude ${ }^{1} \mathrm{H}$ NMR analysis indicated a mixture composed of $69 \%$ yield of the desired chloride (1-B), 7\% yield of (1-T), and complete consumption of starting material (Figure S3).

Spectral data for $\mathbf{1}$ was consistent with reported data. ${ }^{13}$

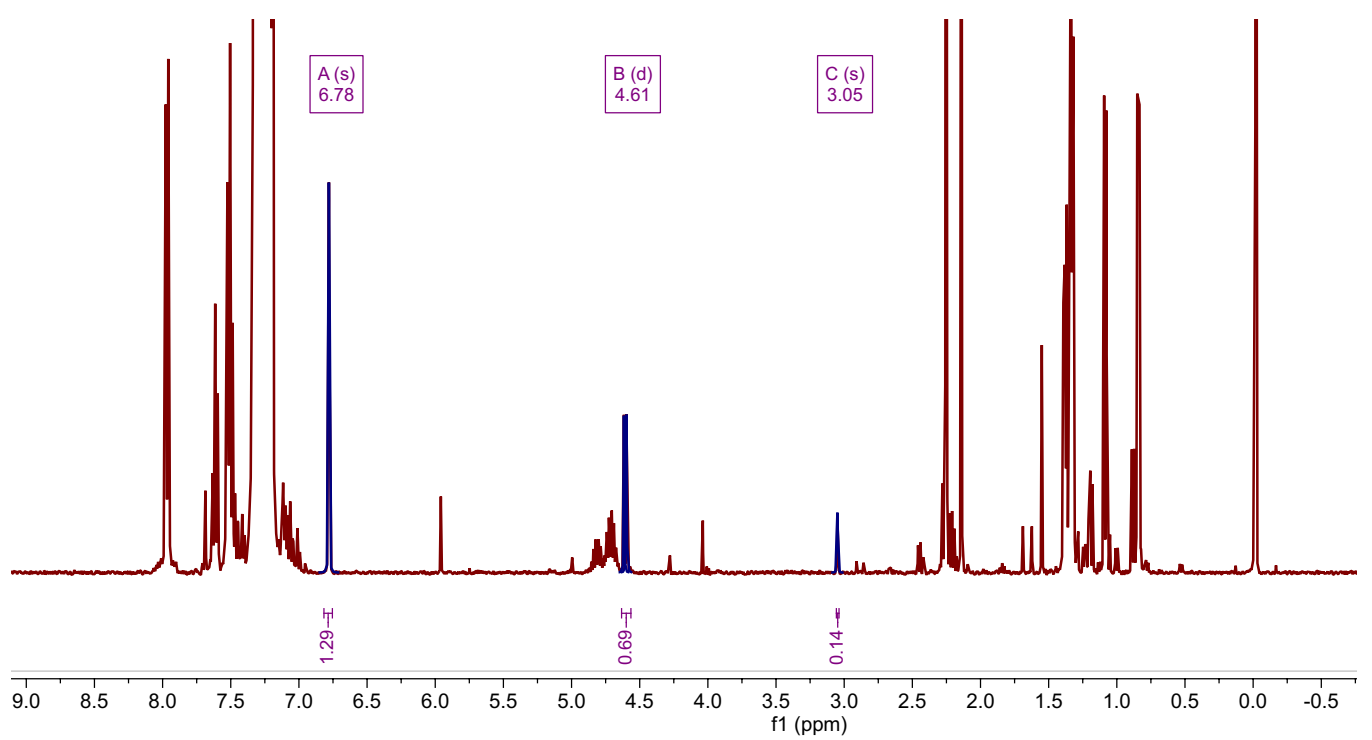

Figure S5. Crude ${ }^{1} \mathrm{H}$ NMR Spectrum $\left(\mathrm{CDCl}_{3}, 400 \mathrm{MHz}, 25^{\circ} \mathrm{C}\right)$ of the reaction mixture for 1 following the addition of $0.215 \mathrm{mmol}(30 \mu \mathrm{L})$ of mesitylene as an external standard $(6.78 \mathrm{ppm})$. The resolved benzylic protons for both 2-B (4.61 ppm, d) and 2-T (3.05ppm, s) chlorides are labeled and integrated. 
(1-chloro-3-methylbutyl)benzene (2-B)<smiles>CC(C)CC(Cl)c1ccccc1</smiles>

Reaction conducted using Procedure $\mathbf{C}$ with isopentylbenzene $(29.6 \mathrm{mg}, 34.7 \mu \mathrm{L}, 0.2 \mathrm{mmol}, 1$ equiv)

Following workup, the obtained residue was purified by column chromatography on silica gel eluting with $100 \%$ heptane to afford a mixture composed of $28.5 \mathrm{mg}$ (78 \% yield) of 2-B and 1.46 $\mathrm{mg}$ (4\% yield) of 2-T as a colorless oil. Spectral data for 2-B. matched those reported in the literature. ${ }^{14}$

NMR Data for 2-B

${ }^{1} \mathrm{H}$ NMR (500 MHz, Chloroform- $d$ ) $\delta 7.42-7.27(\mathrm{~m}, 5 \mathrm{H}), 4.93(\mathrm{dd}, J=8.8,6.3 \mathrm{~Hz}, 1 \mathrm{H}), 2.07$ (ddd, $J=13.9,8.8,6.2 \mathrm{~Hz}, 1 \mathrm{H}), 1.85$ (ddd, $J=14.0,7.6,6.4 \mathrm{~Hz}, 1 \mathrm{H}), 1.79-1.69(\mathrm{~m}, 1 \mathrm{H})$, $0.94(\mathrm{dd}, J=6.6,4.7 \mathrm{~Hz}, 6 \mathrm{H}) \mathrm{ppm}$

${ }^{13} \mathrm{C}$ NMR (126 MHz, Chloroform- $d$ ) $\delta$ 142.1, 128.6, 128.2, 126.9, 62.1, 48.8, 25.6, 22.4, 21.8 ppm. 
<smiles>CC(Cl)c1ccccc1</smiles>

Reaction conducted using Procedure B with ethylbenzene ( $0.2 \mathrm{mmol}, 21.2 \mathrm{mg}, 24.4 \mu \mathrm{L}, 1$ equiv). Following workup, crude ${ }^{1} \mathrm{H}$ NMR analysis indicated the product was isolated as a mixture comprised of $13 \%$ of remaining $\mathrm{C}-\mathrm{H}$ starting material and $91 \%$ of the desired chloride 3 (Figure S4).

Spectral data for $\mathbf{3}$ was consistent with reported data. ${ }^{15}$

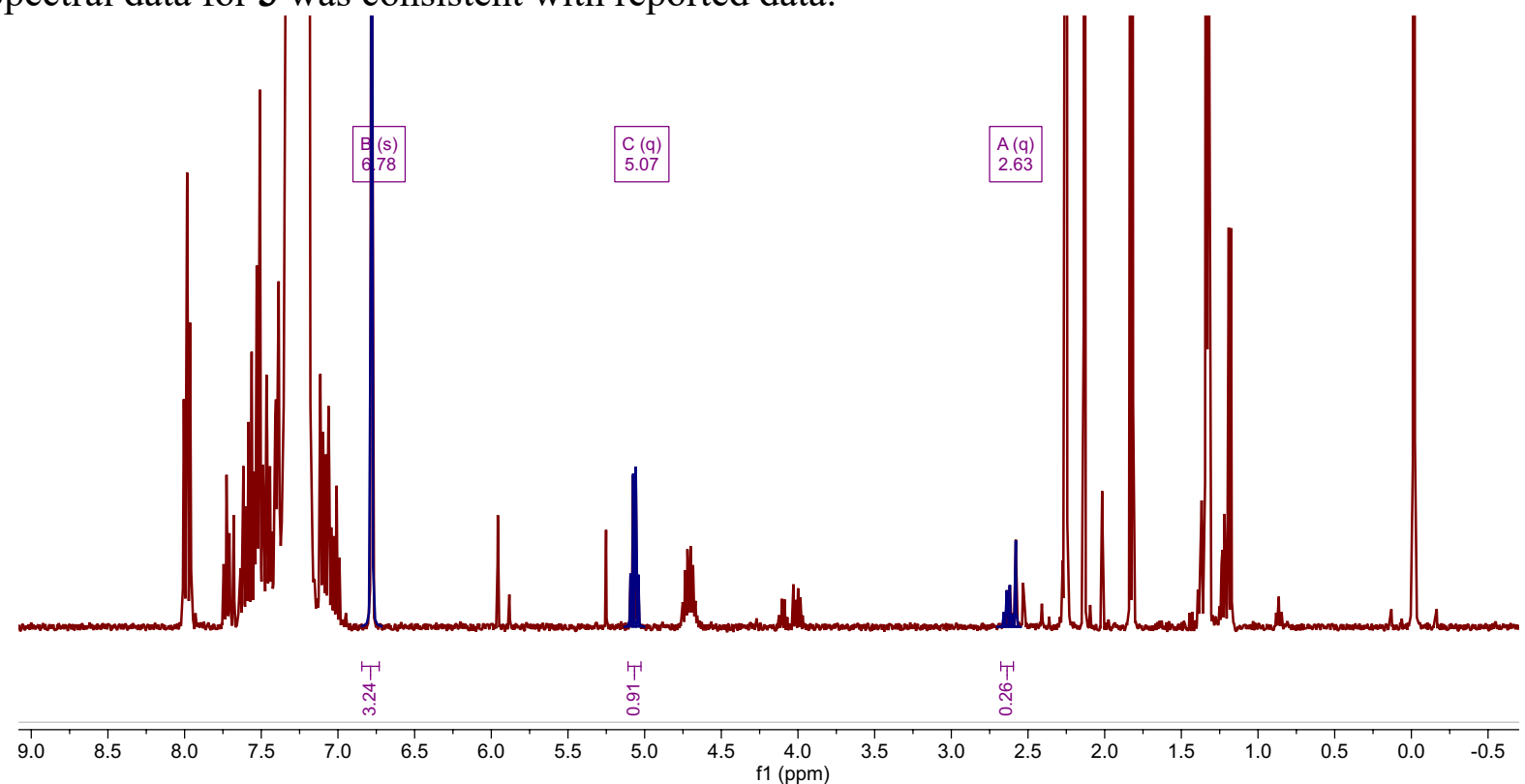

Figure S6. Crude ${ }^{1} \mathrm{H}$ NMR Spectrum $\left(\mathrm{CDCl}_{3}, 400 \mathrm{MHz}, 25^{\circ} \mathrm{C}\right)$ of the reaction mixture for 3 following the addition of $0.215 \mathrm{mmol}(30 \mu \mathrm{L})$ of mesitylene as an external standard $(6.78 \mathrm{ppm})$. The resolved benzylic protons of product $(5.07 \mathrm{ppm})$ and starting material $(2.63 \mathrm{ppm})$ are labeled and integrated. 
<smiles>CC(Cl)c1ccc(C(F)(F)F)cc1</smiles>

Reaction conducted using Procedure G with 4-ethylbenzotrifluoride $(0.5 \mathrm{mmol}, 87.0 \mathrm{mg}, 1$ equiv). Following workup, crude ${ }^{1} \mathrm{H}$ NMR analysis indicated the product was isolated as a mixture comprised of $12 \%$ of remaining $\mathrm{C}-\mathrm{H}$ starting material and $69 \%$ of the desired chloride 4 (Figure S5).

Spectral data for $\mathbf{4}$ was consistent with reported data. ${ }^{16}$

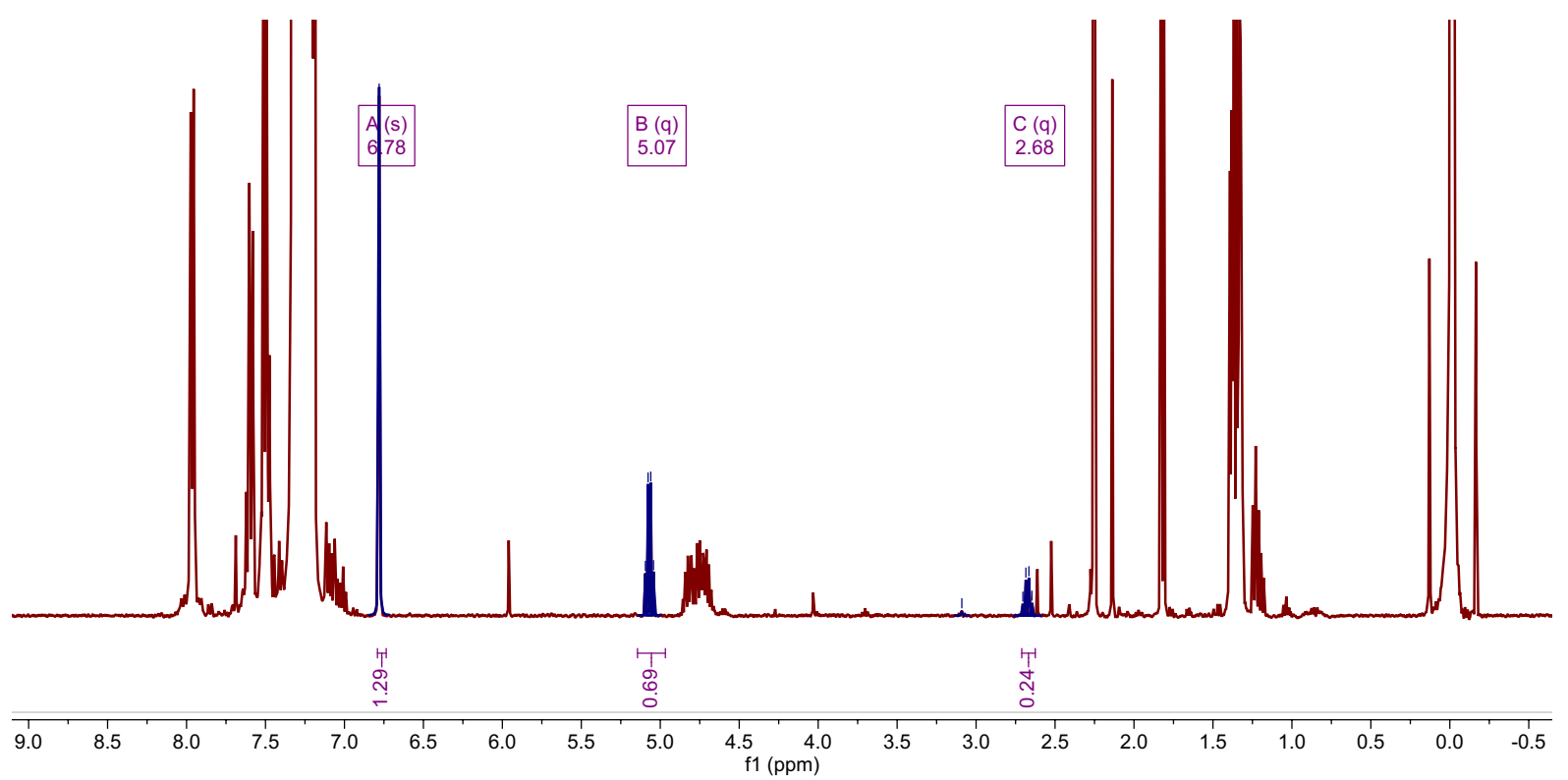

Figure S7. Crude ${ }^{1} \mathrm{H}$ NMR Spectrum $\left(\mathrm{CDCl}_{3}, 400 \mathrm{MHz}, 25^{\circ} \mathrm{C}\right)$ of the reaction mixture for 4 following the addition of $0.215 \mathrm{mmol}(30 \mu \mathrm{L})$ of mesitylene as an external standard $(6.78 \mathrm{ppm})$. The resolved benzylic protons of product $(5.07 \mathrm{ppm})$ and starting material $(2.68 \mathrm{ppm})$ are labeled and integrated. 
<smiles>COc1ccc(C(C)Cl)cc1</smiles>

Reaction conducted using Procedure A with 4-ethylanisole (27.2 mg, $28.4 \mu \mathrm{L}, 0.2 \mathrm{mmol}, 1$ equiv). Following workup, crude ${ }^{1} \mathrm{H}$ NMR analysis indicated $24 \%$ yield of the desired chloride 5, 50\% yield of the sulfonimide side-product, and complete consumption of starting material (Figure S6).

Spectral data for 5 was consistent with reported data. ${ }^{17}$

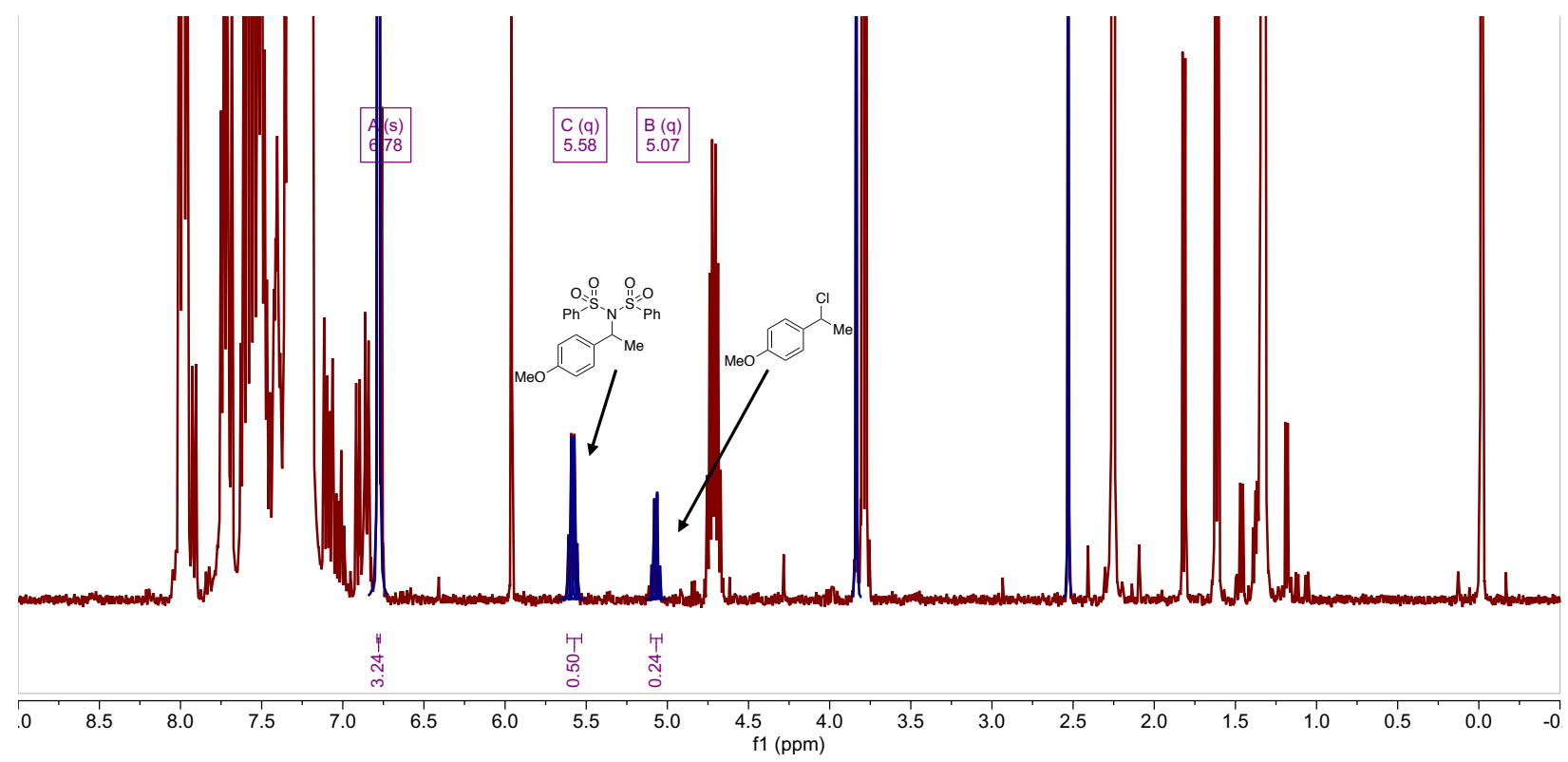

Figure S8. Crude ${ }^{1} \mathrm{H}$ NMR Spectrum $\left(\mathrm{CDCl}_{3}, 400 \mathrm{MHz}, 25^{\circ} \mathrm{C}\right)$ of the reaction mixture for 5 following the addition of $0.215 \mathrm{mmol}(30 \mu \mathrm{L})$ of mesitylene as an external standard $(6.78 \mathrm{ppm})$. The resolved benzylic protons $(5.07 \mathrm{ppm})$ of product and sulfonamide side-product (5.58 ppm) are labeled and integrated. 
(1,3-dichloropropyl)benzene (6)<smiles>ClCCC(Cl)c1ccccc1</smiles>

Reaction conducted using Procedure G with (3-chloropropyl)benzene (77.4 mg, $74.4 \mu \mathrm{L}, 0.5$ mmol, 1 equiv)

The obtained residue was purified by column chromatography on silica gel eluting with 100\% heptane to afford a mixture comprised of $78 \mathrm{mg}$ ( $84 \%$ yield) of 6 and $5.4 \mathrm{mg}$ (7\% recovered) of unreacted $\mathrm{C}-\mathrm{H}$ starting material as a clear light-yellow oil. Spectral data matched those reported in the literature. ${ }^{18}$

NMR Spectroscopy:

${ }^{1} \mathrm{H}$ NMR (400 MHz, Chloroform- $d$ ) $\delta$ 7.43-7.28 (m, 5H), 5.14 (dd, J=9.0, $\left.5.4 \mathrm{~Hz}, 1 \mathrm{H}\right), 3.75$ (ddd, $J=11.1,8.1,5.1 \mathrm{~Hz}, 1 \mathrm{H}), 3.55(\mathrm{ddd}, J=11.1,8.1,5.1 \mathrm{~Hz}, 1 \mathrm{H}), 2.55$ (ddt, $J=14.6,9.0,5.5$ $\mathrm{Hz}, 1 \mathrm{H}), 2.41$ (ddt, $J=14.6,8.1,5.5 \mathrm{~Hz}, 1 \mathrm{H}) \mathrm{ppm}$.

${ }^{13} \mathrm{C}$ NMR (101 MHz, Chloroform- $\left.d\right) \delta 140.5,128.9,128.7,127.0,60.0,42.3,41.8$ ppm.

(3-bromo-1-chloropropyl)benzene (7)<smiles>ClC(CCBr)c1ccccc1</smiles>

Reaction conducted using Procedure G with (3-bromopropyl)benzene (99.5 mg, $75.9 \mu \mathrm{L}, 0.5$ mmol, 1 equiv)

The obtained residue was purified by column chromatography on silica gel eluting with 100\% heptane to afford $94.3 \mathrm{mg}$ ( $81 \%$ yield) of 7 and $7.84 \mathrm{mg}$ (8\% yield) of unreacted $\mathrm{C}-\mathrm{H}$ starting material as a clear light-yellow oil. Spectral data matched those reported in the literature. ${ }^{5}$

NMR Spectroscopy:

${ }^{1} \mathrm{H}$ NMR (500 MHz, Chloroform- $d$ ) $\delta$ 7.45-7.28 (m, 6H), 5.12 (dd, $\left.J=8.8,5.4 \mathrm{~Hz}, 1 \mathrm{H}\right), 3.59$ (ddd, $J=10.3,8.0,5.5 \mathrm{~Hz}, 1 \mathrm{H}), 3.46-3.36(\mathrm{~m}, 1 \mathrm{H}), 2.63$ (ddt, $J=14.7,8.9,5.8 \mathrm{~Hz}, 1 \mathrm{H}), 2.48$ (ddt, $J=14.8,8.0,5.6 \mathrm{~Hz}, 1 \mathrm{H}) \mathrm{ppm}$.

${ }^{13} \mathrm{C}$ NMR (126 MHz, Chloroform- $d$ ) $\delta$ 140.5, 128.9, 128.7, 127.0, 61.1, 42.4, 30.1 ppm. 
3-chloro-3-phenylpropyl acetate (8)<smiles>CC(=O)OCCC(Cl)c1ccccc1</smiles>

Reaction conducted using Procedure G with 3-phenylpropyl acetate ( $89.1 \mathrm{mg}, 0.5 \mathrm{mmol}, 1$ equiv).

The obtained residue was purified by column chromatography on silica gel eluting with a solvent mixture of ethyl acetate:hexanes (10:90 (v:v)) to afford $79.8 \mathrm{mg}$ (75\% yield) of $\mathbf{8}$ as a clear lightyellow oil. Spectral data matched those reported in the literature. ${ }^{5}$

NMR Spectroscopy:

${ }^{1} \mathrm{H}$ NMR (400 MHz, Chloroform- $d$ ) $\delta 7.42-7.30(\mathrm{~m}, 5 \mathrm{H}), 5.00$ (dd, $\left.J=8.7,5.9 \mathrm{~Hz}, 1 \mathrm{H}\right), 4.24$ $(\mathrm{ddd}, J=11.3,7.6,5.4 \mathrm{~Hz}, 1 \mathrm{H}), 4.14(\mathrm{dt}, J=11.4,5.8 \mathrm{~Hz}, 1 \mathrm{H}), 2.50-2.28(\mathrm{~m}, 2 \mathrm{H}), 2.05(\mathrm{~s}$, 3H) ppm.

${ }^{13} \mathrm{C}$ NMR (101 MHz, Chloroform- $d$ ) $\delta$ 170.9, 141.0, 128.8, 128.6, 126.9, 61.6, 59.9, 38.7, 20.9 ppm.

2-(1-chloroethyl)naphthalene (9)<smiles>CC(Cl)c1ccc2ccccc2c1</smiles>

Reaction conducted using Procedure E with 2-ethylnaphthalene (78.1 mg, $78.7 \mu \mathrm{L}, 0.5 \mathrm{mmol}, 1$ equiv).

The crude residue was purified by column chromatography on silica gel eluting with a $100 \%$ heptane to afford $92.5 \mathrm{mg}$ ( $97 \%$ yield) of 9 as a white solid. Spectral data matched those reported in the literature ${ }^{17}$

NMR Spectroscopy:

${ }^{1} \mathrm{H}$ NMR $(500 \mathrm{MHz}$, Chloroform- $d$ ) $\delta 7.88-7.80(\mathrm{~m}, 4 \mathrm{H}), 7.57(\mathrm{dd}, J=8.5,1.8 \mathrm{~Hz}, 1 \mathrm{H}), 7.51-$ $7.45(\mathrm{~m}, 2 \mathrm{H}), 5.27(\mathrm{q}, J=6.8 \mathrm{~Hz}, 1 \mathrm{H}), 1.94(\mathrm{~d}, J=6.8 \mathrm{~Hz}, 3 \mathrm{H}) \mathrm{ppm}$.

${ }^{13} \mathrm{C}$ NMR (126 MHz, Chloroform-d) $\delta$ 140.1, 133.1, 133.1, 128.7, 128.1, 127.7, 126.4, 126.4, $125.2,124.5,59.1,26.4$ ppm. 
Bromo-4-(1-chloropropyl)benzene (10)<smiles>CCC(Cl)c1ccc(Br)cc1</smiles>

Reaction conducted using Procedure $\mathbf{C}$ with 1-bromo-4-propylbenzene (39.8. mg, $30.9 \mu \mathrm{L}, 0.2$ mmol, 1 equiv).

Following workup, the obtained residue was purified by column chromatography on silica gel eluting with $100 \%$ hexanes to afford $35.0 \mathrm{mg}$ (75\% yield) of $\mathbf{1 0}$ as a colorless oil.

NMR Spectroscopy:

${ }^{1} \mathrm{H}$ NMR (500 MHz, Chloroform-d) $\delta 7.47$ (d, 2H), 7.24 (d, 2H), 4.72 (dd, J = 7.9, $\left.6.5 \mathrm{~Hz}, 1 \mathrm{H}\right)$, $2.15-1.97(\mathrm{~m}, 2 \mathrm{H}), 0.97(\mathrm{t}, \mathrm{J}=7.3 \mathrm{~Hz}, 3 \mathrm{H}) \mathrm{ppm}$.

${ }^{13} \mathrm{C}$ NMR (126 MHz, Chloroform- $d$ ) $\delta$ 140.8, 131.8, 128.8, 122.1, 64.5, 33.2, 11.7 ppm.

HRMS (ESI) m/z: [M-Cl]+ Calcd for C9H10Br, 196.9960; Found 196.9961.

Methyl 3-chloro-3-phenylpropanoate (11)<smiles>COC(=O)CC(Cl)c1ccccc1</smiles>

Reaction conducted using Procedure $\mathbf{C}$ with methyl 3-phenylpropanoate $(0.2 \mathrm{mmol}, 32.8 \mathrm{mg}$, $31.6 \mu \mathrm{L}, 1$ equiv)

Following workup, the obtained residue was purified by column chromatography on silica gel eluting with with a solvent mixture of ethyl acetate:hexanes (10:90 (v:v)) to afford $28.6 \mathrm{mg}(72 \%$ yield) of $\mathbf{1 1}$ as a clear colorless oil. Spectral data matched those reported in the literature. ${ }^{19}$

NMR Spectroscopy:

${ }^{1} \mathrm{H}$ NMR (400 MHz, Chloroform-d) $\delta 7.43-7.29(\mathrm{~m}, 5 \mathrm{H}), 5.36(\mathrm{dd}, \mathrm{J}=9.1,5.7 \mathrm{~Hz}, 1 \mathrm{H}), 3.71(\mathrm{~s}$, $3 \mathrm{H}), 3.20(\mathrm{dd}, \mathrm{J}=16.0,9.1 \mathrm{~Hz}, 1 \mathrm{H}), 3.04(\mathrm{dd}, \mathrm{J}=16.0,5.7 \mathrm{~Hz}, 1 \mathrm{H}) \mathrm{ppm}$.

${ }^{13} \mathrm{C}$ NMR (101 MHz, Chloroform-d) $\delta 170.1,140.3,128.8,128.8,126.9,58.0,52.1,44.7$ ppm. 
$N$-(2-chloro-2-phenylethyl)-2,2,2-trifluoroacetamide (12)<smiles>O=C(NCC(Cl)c1ccccc1)C(F)(F)F</smiles>

Reaction conducted using Procedure C with 2,2,2-trifluoro-N-phenethylacetamide (0.2 mmol, $43.4 \mathrm{mg}, 1$ equiv)

Following workup, the obtained residue was purified by column chromatography on silica gel eluting with a solvent mixture of ethyl acetate:hexanes (7:93 (v:v)) to afford $35.2 \mathrm{mg}$ (70\% yield) and $1.74 \mathrm{mg}(4 \%)$ of $\mathrm{C}-\mathrm{H}$ starting material as a white solid.

NMR Spectroscopy:

${ }^{1} \mathrm{H}$ NMR (400 MHz, Chloroform-d) $\delta 7.40-7.30(\mathrm{~m}, 5 \mathrm{H}), 6.64(\mathrm{~s}, 1 \mathrm{H}), 5.01$ (dd, J = 9.0, $4.9 \mathrm{~Hz}$, 1H), 3.94 (ddd, J = 14.3, 7.0, 4.9 Hz, 1H), $3.695(\mathrm{ddd}, \mathrm{J}=14.2,9.0,5.3 \mathrm{~Hz}, 1 \mathrm{H}) \mathrm{ppm}$.

${ }^{13} \mathrm{C}$ NMR (101 MHz, Chloroform-d) $\delta 157.3(\mathrm{q}, \mathrm{J}=37.7 \mathrm{~Hz}), 137.5,129.3,129.1,127.0,114.2$ $(\mathrm{q}, \mathrm{J}=288.2), 60.8,47.1 \mathrm{ppm}$.

${ }^{19}$ F NMR (377 MHz, Chloroform- $d$ ) $\delta-75.92 \mathrm{ppm}$.

HRMS (ESI) m/z: [M+Na]+ Calcd for C10H9ClF3NONa 274.0217; Found 274.0213.

$N$-(3-chloro-3-phenylpropyl)-2,2,2-trifluoroacetamide (13)<smiles>O=C(NCCC(Cl)c1ccccc1)C(F)(F)F</smiles>

13

Reaction conducted using Procedure H with 2,2,2-trifluoro-N-(3-phenylpropyl)acetamide (1.2 mmol, $277.5 \mathrm{mg}, 1$ equiv).

Following workup, the obtained residue was purified by column chromatography on silica gel eluting with a solvent mixture of ethyl acetate:hexanes $(10: 90(\mathrm{v}: \mathrm{v}))$ to afford a mixture comprised of $229.5 \mathrm{mg}$ (72\% yield) of $\mathbf{1 3}$ and $47.2 \mathrm{mg}$ (17\%) of recovered C-H starting material as a white solid.

NMR Spectroscopy:

${ }^{1} \mathrm{H}$ NMR (500 MHz, Chloroform- $d$ ) $\delta 7.42-7.25$ (m, 5H), 6.41 (s, 1H), 4.92 (dd, J= 7.7, 6.6 Hz, $1 \mathrm{H}), 3.66-3.44(\mathrm{~m}, 2 \mathrm{H}), 2.43-2.27(\mathrm{~m}, 2 \mathrm{H}) \mathrm{ppm}$.

${ }^{13} \mathrm{C}$ NMR (126 MHz, Chloroform- $d$ ) $\delta 157.4(\mathrm{q}, J=37.1 \mathrm{~Hz}), 140.5,129.0,128.9,126.8,115.7$ $(\mathrm{q}, J=287.8 \mathrm{~Hz}), 60.9,38.7,37.9 \mathrm{ppm}$.

${ }^{19} \mathrm{~F}$ NMR (377 MHz, Chloroform- $d$ ) $\delta-75.96 \mathrm{ppm}$.

HRMS (ESI) m/z: [M-H]- Calcd for C11H10ClF3NO 264.0409; Found 264.0409. 
$N$-(4-chloro-4-phenylbutyl)-2,2,2-trifluoroacetamide (14)<smiles>O=C(NCCCC(Cl)c1ccccc1)C(F)(F)F</smiles>

14

Reaction conducted using Procedure $\mathbf{C}$ with 2,2,2-trifluoro- $N$-(4-phenylbutyl)acetamide $(0.2$ mmol, $49.1 \mathrm{mg}, 1$ equiv)

Following workup, the obtained residue was purified by column chromatography on silica gel eluting with a solvent mixture of ethyl acetate:hexanes $(15: 85(\mathrm{v}: \mathrm{v}))$ to afford a mixture of 14, C$\mathrm{H}$ starting material, and diisopropyl chlorophosphate. The mixture was further purified by preparatory thin layer chromatography eluting with a solvent mixture of ethyl acetate:hexanes $(10: 90(\mathrm{v}: \mathrm{v}))$ to afford $41.4 \mathrm{mg}(\mathbf{7 4 \%})$ of $\mathbf{1 4}$ as a white solid.

NMR Spectroscopy:

${ }^{1} \mathrm{H}$ NMR (500 MHz, Chloroform- $d$ ) $\delta$ 7.39-7.29 (m, 5H), $6.27(\mathrm{~s}, 1 \mathrm{H}), 4.88(\mathrm{dd}, J=8.4,5.9 \mathrm{~Hz}$, $1 \mathrm{H}), 3.46-3.34(\mathrm{~m}, 2 \mathrm{H}), 2.22-2.12(\mathrm{~m}, 1 \mathrm{H}), 2.11-2.02(\mathrm{~m}, 1 \mathrm{H}), 1.88-1.75(\mathrm{~m}, 1 \mathrm{H}), 1.73-$ $1.60(\mathrm{~m}, 1 \mathrm{H}) \mathrm{ppm}$.

${ }^{13} \mathrm{C}$ NMR $(126 \mathrm{MHz}$, Chloroform- $d$ ) $\delta 157.1(\mathrm{q}, J=37.0 \mathrm{~Hz}), 141.1,128.8,128.5,115.8(\mathrm{q}, J=$ $288.0 \mathrm{~Hz}), 62.8,39.3,37.0,26.7 \mathrm{ppm}$.

${ }^{19} \mathrm{~F}$ NMR (377 MHz, Chloroform-d) $\delta-75.90 \mathrm{ppm}$.

HRMS (ESI) m/z: [M+Na]+ Calcd for C12H13ClF3NONa 302.05300; Found 302.0527.

2-bromo-9-chloro-9H-fluorene (15)

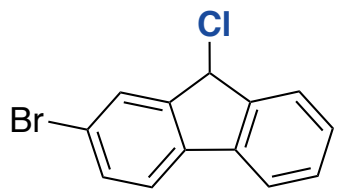

15

Reaction conducted using Procedure E with 2-bromo-9H-fluorene (0.5 mmol, $122.6 \mathrm{mg}, 1$ equiv)

Following workup, the obtained residue was purified by column chromatography on silica gel eluting with $100 \%$ heptane to afford $110.4 \mathrm{mg}$ (79\% yield) of $\mathbf{1 5}$ as a white solid.

NMR Spectroscopy:

${ }^{1} \mathrm{H}$ NMR (500 MHz, Chloroform- $\left.d\right) \delta 7.81(\mathrm{~s}, 1 \mathrm{H}), 7.67(\mathrm{t}, J=7.1 \mathrm{~Hz}, 2 \mathrm{H}), 7.57(\mathrm{~d}, J=1.2 \mathrm{~Hz}$, 2H), 7.47-7.38 (m, 2H), $5.79(\mathrm{~s}, 1 \mathrm{H}) \mathrm{ppm}$.

${ }^{13} \mathrm{C}$ NMR (126 MHz, Chloroform-d) $\delta$ 145.8, 143.5, 139.0, 132.5, 129.6, 129.2, 128.4, 125.9, 121.6, 121.4, 120.2, $56.8 \mathrm{ppm}$.

HRMS (ESI) m/z: [M-Cl]+ Calcd for C13H8Br 242.9804; Found 242.9804. 
6-bromo-4-chlorochromane (16)<smiles>ClC1CCOc2ccc(Br)cc21</smiles>

Reaction conducted using Procedure F with 6-bromochroman (0.5 mmol, $106.5 \mathrm{mg}, 1$ equiv)

Following workup, the obtained residue was purified by column chromatography on silica gel luting with ethyl acetate:hexanes (5:95 (v:v)) to afford $92.8 \mathrm{mg}$ (75\% yield) of 16 as a colorless oil.

NMR Spectroscopy:

${ }^{1} \mathrm{H}$ NMR (400 MHz, Chloroform- $d$ ) $\delta 7.43(\mathrm{~d}, J=2.5 \mathrm{~Hz}, 1 \mathrm{H}), 7.34-7.27(\mathrm{~m}, 1 \mathrm{H}), 6.73(\mathrm{~d}, J=$ $8.8 \mathrm{~Hz}, 1 \mathrm{H}), 5.17(\mathrm{t}, J=3.7 \mathrm{~Hz}, 1 \mathrm{H}), 4.45(\mathrm{td}, J=11.5,2.2 \mathrm{~Hz}, 1 \mathrm{H}), 4.39-4.28(\mathrm{~m}, 1 \mathrm{H}), 2.49$ $2.39(\mathrm{~m}, 1 \mathrm{H}), 2.34-2.26(\mathrm{~m}, 1 \mathrm{H}) \mathrm{ppm}$.

${ }^{13} \mathrm{C}$ NMR (101 MHz, Chloroform- $\left.d\right) \delta$ 153.2, 133.1, 133.1, 124.0, 119.3, 112.5, 61.8, 51.9, 31.6 ppm.

HRMS (ESI) m/z: [M+H]+ Calcd for C9H9BrClO 245.9442; Found 245.043.

4-chloro-3,4-dihydronaphthalen-1(2H)-one (17)<smiles>O=C1CCC(Cl)c2ccccc21</smiles>

17

Reaction conducted using Procedure F with 3,4-dihydronaphthalen-1(2H)-one (0.5 mmol, 73.1 mg, 1 equiv)

Following workup, the obtained residue was purified by column chromatography on silica gel eluting with ethyl acetate:hexanes (10:90 (v:v)) to afford $54.2 \mathrm{mg}$ (60\% yield) of $\mathbf{1 7}$ as a light yellow oil. Spectral data matched those reported in the literature. ${ }^{5}$

NMR Spectroscopy:

${ }^{1} \mathrm{H}$ NMR (400 MHz, Chloroform- $d$ ) $\delta 8.05(\mathrm{~d}, J=7.8,1 \mathrm{H}), 7.62-7.55(\mathrm{~m}, 1 \mathrm{H}), 7.51-7.41(\mathrm{~m}$, $2 \mathrm{H}), 5.38(\mathrm{t}, J=3.8 \mathrm{~Hz}, 1 \mathrm{H}), 3.16(\mathrm{~m}, 1 \mathrm{H}), 2.81-2.42(\mathrm{~m}, 3 \mathrm{H}) \mathrm{ppm}$.

${ }^{13} \mathrm{C}$ NMR (101 MHz, Chloroform- $d$ ) $\delta$ 196.3, 142.2, 134.2, 131.1, 129.3, 129.0, 127.4, 56.6, 33.96, $31.8 \mathrm{ppm}$. 
1-(6-(tert-butyl)-3-chloro-1,1-dimethyl-2,3-dihydro-1H-inden-4-yl)ethan-1-one (18) and 1-(6(tert-butyl)-3-hydroxy-1,1-dimethyl-2,3-dihydro-1 $H$-inden-4-yl)ethan-1-one (18-OH)<smiles>CC(=O)c1cc(C(C)(C)C)cc2c1C(Cl)CC2(C)C</smiles>

18

\section{Quantitative ( ${ }^{1} \mathrm{H}-\mathrm{NMR}$ Yield)}<smiles>CC(=O)c1cc(C(C)(C)C)cc2c1C(O)CC2(C)C</smiles>

$18 a$

Reaction conducted using Procedure A with 1-(6-(tert-butyl)-1,1-dimethyl-2,3-dihydro-1Hinden-4-yl)ethan-1-one ( $0.2 \mathrm{mmol}, 48.9 \mathrm{mg}, 1$ equiv) Due to the instability of 18, it was converted and isolated as the alcohol.

Work Up/Hydroxylation: After $16 \mathrm{~h}$, mesitylene $(0.215 \mathrm{mmol}, 30 \mu \mathrm{L})$ was added to the greencolored heterogenous solution as an external NMR standard. The crude reaction mixture was filtered through a pad of celite directly into a $10 \mathrm{~mL}$ test tube using $\mathrm{CHCl}_{3}$. An aliquot $(30 \mu \mathrm{L})$ from the now clear light-green solution was taken for NMR analysis to detect formation of product and consumption of starting material. After NMR analysis, the crude reaction mixture was concentrated on the rotovap at room temperature to remove solvent (chlorobenzene and chloroform). NMR analysis indicated quantitative yield of the desired chloride (Figure S7). To the concentrated residue was added $\mathrm{SiO}_{2}\left(0.6 \mathrm{mmol}, 36.0 \mathrm{mg}, 3\right.$ equiv) and $\mathrm{H}_{2} \mathrm{O}$ :Acetone (1:1 $(\mathrm{v}: \mathrm{v}), 1 \mathrm{~mL}, \mathrm{c}=0.2 \mathrm{M})$. The reaction mixture was stirred for $1 \mathrm{~h}$ on an aluminum heat block at 30 ${ }^{\circ} \mathrm{C}$. The reaction mixture was concentrated on the rotovap and purified by column chromatography on silica gel eluting with a solvent mixture of ethyl acetate:hexanes (10:90 (v:v)) to afford 49.4 $\mathrm{mg}$ of 18-OH as a colorless solid (95\% yield). Spectral data matched those reported in the literature. ${ }^{20}$

NMR Spectroscopy for 18a

${ }^{1} \mathrm{H}$ NMR (600 MHz, Chloroform- $\left.d\right) \delta 7.77(\mathrm{~d}, J=1.7 \mathrm{~Hz}, 1 \mathrm{H}), 7.43(\mathrm{~d}, J=1.7 \mathrm{~Hz}, 1 \mathrm{H}), 5.38$ (ddd, $J=7.6,3.9,1.9 \mathrm{~Hz}, 1 \mathrm{H}), 4.52(\mathrm{~d}, J=2.0 \mathrm{~Hz}, 1 \mathrm{H}), 2.68(\mathrm{~s}, 3 \mathrm{H}), 2.29(\mathrm{dd}, J=13.5,7.6 \mathrm{~Hz}, 1 \mathrm{H})$, $2.05(\mathrm{dd}, J=13.5,3.9 \mathrm{~Hz}, 1 \mathrm{H}), 1.40(\mathrm{~s}, 3 \mathrm{H}), 1.37$ (s, 8H), $1.26(\mathrm{~s}, 3 \mathrm{H}) \mathrm{ppm}$.

${ }^{13} \mathrm{C}$ NMR (151 MHz, Chloroform- $d$ ) $\delta 202.8,154.4,152.3,142.3,133.7,126.7,124.9,73.2,48.8$, 42.9, 34.9, 31.4, 31.0, 29.9, 28.0 ppm. 


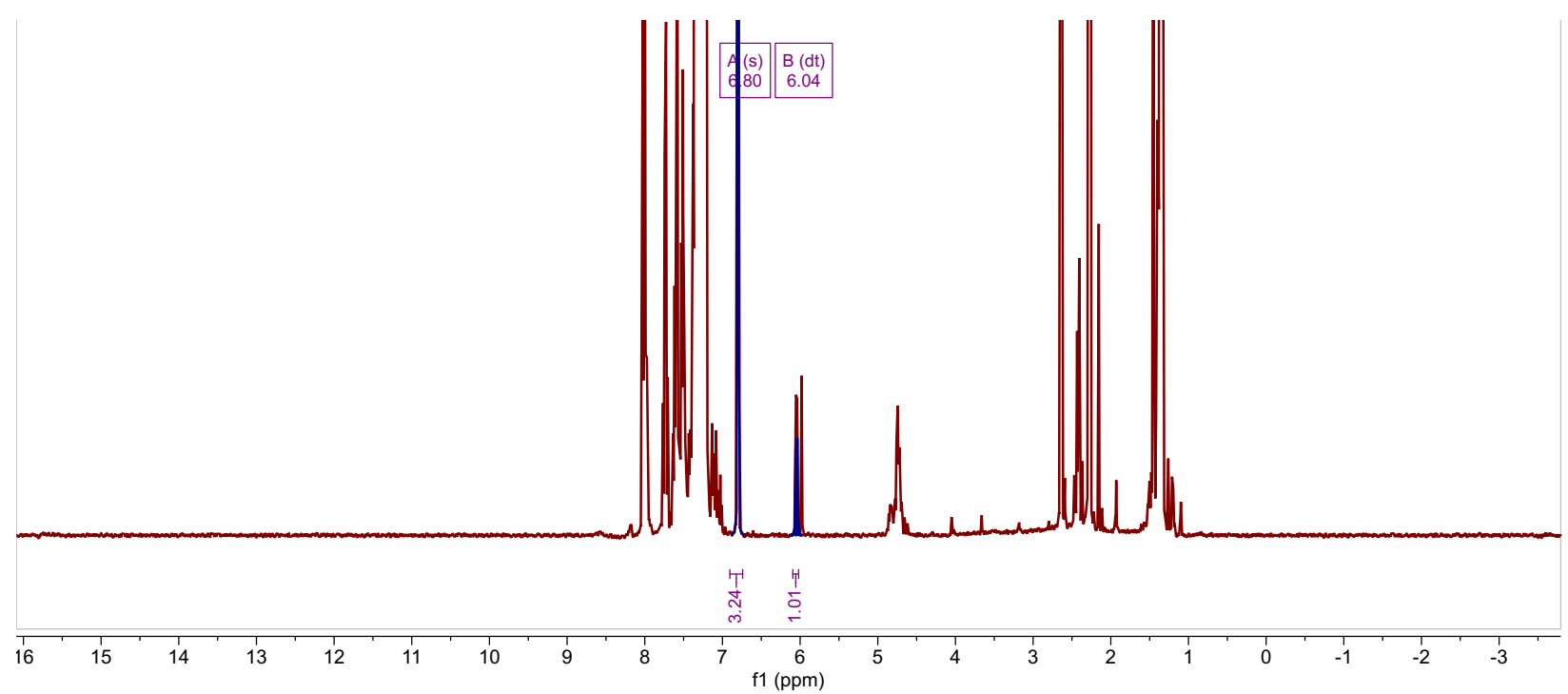

Figure S9. Crude ${ }^{1} \mathrm{H}$ NMR Spectrum $\left(\mathrm{CDCl}_{3}, 400 \mathrm{MHz}, 25^{\circ} \mathrm{C}\right)$ of the reaction mixture for 18 following the addition of $0.215 \mathrm{mmol}(30 \mu \mathrm{L})$ of mesitylene as an external standard $(6.80 \mathrm{ppm})$. The resolved benzylic protons of product $(6.04 \mathrm{ppm})$ are labeled and integrated.

Methyl 2-(4-(1-chloro-2-methylpropyl)phenyl)propanoate (19)<smiles>COC(=O)C(C)c1ccc(C(Cl)C(C)C)cc1</smiles>

19

Reaction conducted using Procedure $\mathbf{C}$ with methyl 2-(4-isobutylphenyl)propanoate (0.2 mmol, $44.0 \mathrm{mg}, 1$ equiv)

Following workup, the obtained residue was purified by column chromatography on silica gel eluting with ethyl acetate:hexanes (10:90 (v:v)) to afford $26.5 \mathrm{mg}$ (52\% yield) of 19 as a colorless oil. Spectral data matched those reported in the literature. ${ }^{21}$

NMR Spectroscopy:

${ }^{1} \mathrm{H}$ NMR (400 MHz, Chloroform- $\left.d\right) \delta 7.34-7.18(\mathrm{~m}, 5 \mathrm{H}), 4.62(\mathrm{~d}, J=7.6 \mathrm{~Hz}, 1 \mathrm{H}), 3.72(\mathrm{q}, J=$ $7.2 \mathrm{~Hz}, 1 \mathrm{H}), 3.66(\mathrm{~s}, 3 \mathrm{H}), 2.28-2.17(\mathrm{~m}, 1 \mathrm{H}), 1.49$ (d, $J=7.2 \mathrm{~Hz}, 3 \mathrm{H}), 1.10(\mathrm{~d}, J=6.6 \mathrm{~Hz}$, $3 \mathrm{H}), 0.87(\mathrm{~d}, J=6.7 \mathrm{~Hz}, 3 \mathrm{H}) \mathrm{ppm}$.

${ }^{13} \mathrm{C}-\mathrm{NMR}$ (101 MHz, Chloroform-d) $\delta 174.9,140.2$, 139.9, 127.7, 127.6, 70.5, 52.09, 45.1, 36.6, 20.2, 19.6, 18.6 ppm. 
(2-bromo-5-(1-chloroethyl)pyridine (20)<smiles>CC(Cl)c1ccc(Br)nc1</smiles>

20

Reaction conducted using Procedure D with 2-bromo-5-ethylpyridine (0.2 mmol, $37.8 \mathrm{mg}, 1$ equiv)

Following workup, the obtained residue was purified by column chromatography on silica gel eluting with ethyl acetate:hexanes $(5: 95(\mathrm{v}: \mathrm{v}))$ to afford $14.9 \mathrm{mg}$ (34\% yield) of 21 as a colorless oil.

NMR Spectroscopy:

${ }^{1} \mathrm{H}$ NMR $(500 \mathrm{MHz}$, Chloroform- $d) \delta 8.38(\mathrm{~d}, J=2.6 \mathrm{~Hz}, 1 \mathrm{H}), 7.65(\mathrm{dd}, J=8.2,2.6 \mathrm{~Hz}, 1 \mathrm{H}), 7.53$ $-7.41(\mathrm{~m}, 1 \mathrm{H}), 5.06(\mathrm{q}, J=6.8 \mathrm{~Hz}, 1 \mathrm{H}), 1.84(\mathrm{~d}, J=6.9 \mathrm{~Hz}, 3 \mathrm{H}) \mathrm{ppm}$.

${ }^{13} \mathrm{C}$ NMR (126 MHz, Chloroform- $d$ ) $\delta$ 148.2, 141.8, 137.8, 128.2, 54.7, $26.2 \mathrm{ppm}$.

HRMS (ESI) m/z: [M+H]+ Calcd for C7H8BrClN 219.9523; Found 219.9523.

3-(2-chloro-2-(3-chlorophenyl)ethyl)picolinonitrile (21)<smiles>N#Cc1ncccc1CC(Cl)c1cccc(Cl)c1</smiles>

Reaction conducted using Procedure D with 3-(3-chlorophenethyl)picolinonitrile (0.2 mmol, 48.5 mg, 1 equiv)

Following workup, the obtained residue was purified by column chromatography on silica gel eluting with hexane: ethyl acetate (90:10 (v:v)) to afford $21.2 \mathrm{mg}$ (38\% yield) of 21 and $5.82 \mathrm{mg}$ $(12 \%)$ of remaining starting material as a yellow solid.

NMR Spectroscopy:

${ }^{1} \mathrm{H}$ NMR $(500 \mathrm{MHz}$, Chloroform- $d$ ) $\delta 8.64(\mathrm{dd}, J=4.7,1.6 \mathrm{~Hz}, 1 \mathrm{H}), 7.69(\mathrm{dd}, J=8.0,1.6 \mathrm{~Hz}, 1 \mathrm{H})$, 7.47 (dd, $J=8.0,4.7 \mathrm{~Hz}, 1 \mathrm{H}), 7.45$ (q, $J=1.5 \mathrm{~Hz}, 1 \mathrm{H}), 7.34-7.29(\mathrm{~m}, 3 \mathrm{H}), 5.12$ (dd, $J=9.4$, $5.1 \mathrm{~Hz}, 1 \mathrm{H}), 3.57(\mathrm{dd}, J=14.5,5.0 \mathrm{~Hz}, 1 \mathrm{H}), 3.48(\mathrm{dd}, J=14.5,9.4 \mathrm{~Hz}, 1 \mathrm{H}) \mathrm{ppm}$.

${ }^{13} \mathrm{C}$ NMR (126 MHz, Chloroform- $d$ ) $\delta$ 149.8, 141.8, 138.8, 137.7, 134.8, 134.4, 130.2, 129.1, $127.1,126.5,125.1,116.0,61.2,43.3 \mathrm{ppm}$.

HRMS (ESI) m/z: [M+H]+ Calcd for C14H11Cl2N2; 277.0293 Found 277.0291. 
4-chloro-1-((4-chloro-3-nitrophenyl)sulfonyl)-1,2,3,4-tetrahydroquinoline (22)<smiles>O=[N+]([O-])c1cc(S(=O)(=O)N2CCC(Cl)c3ccccc32)ccc1Cl</smiles>

Reaction conducted using Procedure A with 1-((4-chloro-3-nitrophenyl)sulfonyl)-1,2,3,4tetrahydroquinoline ( $0.2 \mathrm{mmol}, 70.5 \mathrm{mg}, 1$ equiv)

Following workup, the obtained residue was purified by column chromatography on silica gel eluting with ethyl acetate:hexanes $(5: 95(\mathrm{v}: \mathrm{v}))$ to afford $31.0 \mathrm{mg}(40 \%$ yield) of 22 as a colorless oil.

NMR Spectroscopy:

${ }^{1} \mathrm{H}$ NMR $(500 \mathrm{MHz}$, Chloroform- $d) \delta 8.14(\mathrm{~d}, J=2.1 \mathrm{~Hz}, 1 \mathrm{H}), 7.87(\mathrm{dd}, J=8.4,1.1 \mathrm{~Hz}, 1 \mathrm{H}), 7.71$ $(\mathrm{dd}, J=8.5,2.2 \mathrm{~Hz}, 1 \mathrm{H}), 7.60(\mathrm{~d}, J=8.5 \mathrm{~Hz}, 1 \mathrm{H}), 7.34(\mathrm{ddd}, J=8.6,7.3,1.7 \mathrm{~Hz}, 1 \mathrm{H}), 7.29$ $(\mathrm{dd}, J=7.7,1.7 \mathrm{~Hz}, 1 \mathrm{H}), 7.18(\mathrm{td}, J=7.6,1.2 \mathrm{~Hz}, 1 \mathrm{H}), 5.07(\mathrm{t}, J=3.6 \mathrm{~Hz}, 1 \mathrm{H}), 4.24(\mathrm{dtd}, J=$ 13.1, 3.8, 3.2, $1.0 \mathrm{~Hz}, 1 \mathrm{H}), 2.25(\mathrm{dq}, J=14.7,3.4 \mathrm{~Hz}, 1 \mathrm{H}), 2.12$ (ddt, $J=14.7,12.1,4.3 \mathrm{~Hz}$, 1H) ppm.

${ }^{13} \mathrm{C}$ NMR (126 MHz, Chloroform- $d$ ) $\delta$ 147.7, 138.7, 134.9, 132.9, 132.1, 131.0, 130.6, 130.0, $128.9,125.7,124.5,123.3,54.1,42.5,31.0 \mathrm{ppm}$.

HRMS (ESI) m/z: [M+H]+ Calcd for C15H13Cl2N2O4S; 386.9968; Found 386.9961.

(2-(1-chloroethyl)benzofuran-3-yl)(3,5-dibromo-4-methoxyphenyl)methanone (23)

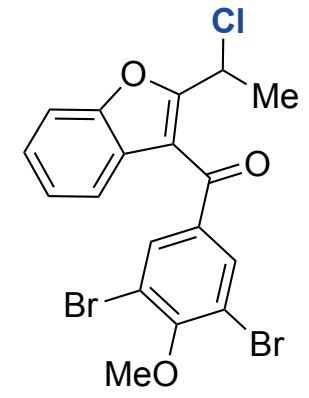

23

Reaction conducted using Procedure B with (3,5-dibromo-4-methoxyphenyl)(2-ethylbenzofuran3-yl)methanone ( $0.2 \mathrm{mmol}, 87.6 \mathrm{mg}, 1$ equiv)

Following workup, the obtained residue was purified by column chromatography on silica gel eluting with hexane: ethyl acetate $(93: 7(\mathrm{v}: \mathrm{v}))$ to afford $64.3 \mathrm{mg}$ (68\% yield) of 23 and $7 \mathrm{mg}(8 \%)$ of elimination side product as a colorless solid. 
NMR Spectroscopy:

${ }^{1} \mathrm{H}$ NMR (500 MHz, Chloroform- $d$ ) $\delta 8.02(\mathrm{~s}, 2 \mathrm{H}), 7.60-7.51(\mathrm{~m}, 1 \mathrm{H}), 7.42-7.34(\mathrm{~m}, 2 \mathrm{H}), 7.26$ $(\mathrm{td}, J=7.4,1.0 \mathrm{~Hz}, 1 \mathrm{H}), 5.36(\mathrm{q}, J=6.9 \mathrm{~Hz}, 1 \mathrm{H}), 3.96(\mathrm{~s}, 3 \mathrm{H}), 1.93(\mathrm{~d}, J=6.9 \mathrm{~Hz}, 3 \mathrm{H}) \mathrm{ppm}$.

${ }^{13} \mathrm{C}$ NMR (126 MHz, Chloroform-d) $\delta$ 186.2, 159.3, 157.3, 153.0, 135.2, 132.9, 125.2, 124.5, 123.4, 120.7, 117.7, 115.0, 110.9, 59.9, 47.5, $22.1 \mathrm{ppm}$.

HRMS (ESI) m/z: [M+H]+ Calcd for C18H14Br2ClO3 470.8993; Found 470.8997.

(2-(1-chlorobutyl)-5-nitrobenzofuran-3-yl)(4-methoxyphenyl)methanone (24)

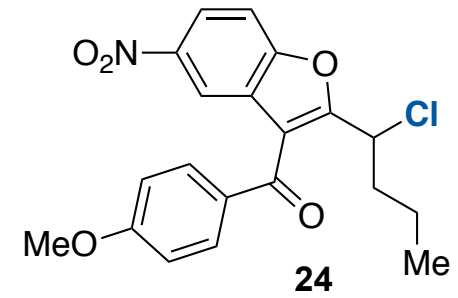

Reaction conducted using Procedure H with (2-butyl-5-nitrobenzofuran-3-yl)(4methoxyphenyl)methanone (1.2 mmol, $424.0 \mathrm{mg}, 1$ equiv)

Following workup, the obtained residue was purified by column chromatography on silica gel eluting with hexane: ethyl acetate $(90: 10(\mathrm{v}: \mathrm{v}))$ to afford $414.2 \mathrm{mg}$ (89\% yield) of 24 as a sticky/oily residue.

NMR Spectroscopy:

${ }^{1} \mathrm{H}$ NMR (500 MHz, Chloroform- $\left.d\right) \delta 8.38(\mathrm{~d}, J=2.3 \mathrm{~Hz}, 1 \mathrm{H}), 8.31(\mathrm{dd}, J=9.0,2.4 \mathrm{~Hz}, 1 \mathrm{H}), 7.89$ $(\mathrm{d}, J=8.8 \mathrm{~Hz}, 1 \mathrm{H}), 7.67(\mathrm{~d}, J=9.1 \mathrm{~Hz}, 1 \mathrm{H}), 7.02(\mathrm{~d}, J=8.8 \mathrm{~Hz}, 2 \mathrm{H}), 5.21(\mathrm{t}, J=7.6 \mathrm{~Hz}, 1 \mathrm{H})$, $3.93(\mathrm{~s}, 3 \mathrm{H}), 2.35-2.16(\mathrm{~m}, 3 \mathrm{H}), 1.53-1.45(\mathrm{~m}, 1 \mathrm{H}), 1.33(\mathrm{ddt}, J=13.5,8.2,6.8 \mathrm{~Hz}, 1 \mathrm{H})$, $0.92(\mathrm{t}, J=7.4 \mathrm{~Hz}, 3 \mathrm{H}) \mathrm{ppm}$.

${ }^{13} \mathrm{C}$ NMR (126 MHz, Chloroform- $d$ ) $\delta 187.9,164.5,161.2,156.5,144.9,132.0,130.3,127.0$, 121.6, 118.6, 118.4, 114.3, 112.2, 55.7, 52.4, 38.5, 20.0, $13.2 \mathrm{ppm}$.

HRMS (ESI) m/z: [M+Na]+ Calcd for C20H18CINO5Na 410.0766; Found 410.0766. 
4-(1-chloropentyl)phenyl 4-methoxybenzoate methoxybenzoate (25)<smiles>COc1ccc(C(=O)Oc2ccc(C([14CH3])Cl)cc2)cc1</smiles>

Reaction conducted using Procedure B with 4-pentylphenyl 4-methoxybenzoate (0.2 mmol, 59.6 mg, 1 equiv)

Following workup, the obtained residue was purified by column chromatography on silica gel eluting with hexane: ethyl acetate (90:10 (v:v)) to afford $54.6 \mathrm{mg}$ (82\% yield) of 25 as a white solid.

NMR Spectroscopy:

${ }^{1} \mathrm{H}$ NMR (500 MHz, Chloroform-d) $\delta 8.15(\mathrm{~d}, J=8.9 \mathrm{~Hz}, 1 \mathrm{H}), 7.43(\mathrm{~d}, J=8.6 \mathrm{~Hz}, 1 \mathrm{H}), 7.20(\mathrm{~d}, J$ $=8.6 \mathrm{~Hz}, 2 \mathrm{H}), 6.99(\mathrm{~d}, J=8.9 \mathrm{~Hz}, 2 \mathrm{H}), 4.88(\mathrm{dd}, J=8.1,6.5 \mathrm{~Hz}, 1 \mathrm{H}), 3.90(\mathrm{~s}, 3 \mathrm{H}), 2.18-$ 2.09 (m, 1H), 2.05 (dddd, $J=13.8,9.9,6.5,5.2 \mathrm{~Hz}, 1 \mathrm{H}), 0.91$ (t, $J=7.1 \mathrm{~Hz}, 3 \mathrm{H}) \mathrm{ppm}$.

${ }^{13} \mathrm{C}$ NMR (126 MHz, Chloroform- $d$ ) $\delta$ 164.77, 163.99, 150.75, 139.46, 132.33, 128.10, 121.90, $121.74,113.89,63.24,55.54,39.84,29.21,22.14,13.91 \mathrm{ppm}$.

HRMS (ESI) m/z: [M+Na]+ Calcd for C19H21ClO3Na 355.10714; Found 355.106.

2-chloro-1,2-bis(4-methoxyphenyl)ethan-1-one (26)<smiles>COc1ccc(C(=O)C(Cl)c2ccc(OC)cc2)cc1</smiles>

Reaction conducted using Procedure A with 1,2-bis(4-methoxyphenyl)ethan-1-one (0.2 mmol, $51.3 \mathrm{mg}, 1$ equiv)

Following workup, the obtained residue was purified by column chromatography on silica gel eluting with dichloromethane: ethyl acetate (90:10 (v:v)) to afford $48.2 \mathrm{mg}$ (83\% yield) of 27 as a colorless oil. Spectral data matched those reported in the literature. ${ }^{22}$

NMR Spectroscopy:

${ }^{1} \mathrm{H}$ NMR (400 MHz, Chloroform- $d$ ) $\delta 7.98-7.90(\mathrm{~m}, 2 \mathrm{H}), 7.44-7.36(\mathrm{~m}, 2 \mathrm{H}), 6.93$ - 6.85 (m, $4 \mathrm{H}), 6.28(\mathrm{~s}, 1 \mathrm{H}), 3.84(\mathrm{~s}, 3 \mathrm{H}), 3.79(\mathrm{~s}, 3 \mathrm{H}) \mathrm{ppm}$.

${ }^{13} \mathrm{C}$ NMR (101 MHz, Chloroform-d) $\delta$ 190.0, 163.9, 160.1, 131.5, 129.8, 128.3, 127.2, 114.6, $114.0,62.1,55.5,55.3 \mathrm{ppm}$. 
<smiles>FC(F)(F)NCCC(Oc1ccc(C(F)(F)F)cc1)c1ccccc1</smiles>

27

Reaction conducted using Procedure I with p 4-Trifluoromethylphenol (0.4 mmol, $64.8 \mathrm{mg} 2$ equiv) Following workup, the obtained residue was purified by column chromatography on silica gel eluting with eluting with a solvent mixture of ethyl acetate:hexanes (08:92 (v:v)) to afford $39.13 \mathrm{mg}$ of 27 as a light-yellow oil (50\% yield).

NMR Spectroscopy:

${ }^{1} \mathrm{H}$ NMR $\left(500 \mathrm{MHz}, \mathrm{CDCl}_{3}\right) \delta 7.46(\mathrm{~d}, J=8.7 \mathrm{~Hz}, 2 \mathrm{H}), 7.39-7.34(\mathrm{~m}, 2 \mathrm{H}), 7.32-7.28(\mathrm{~m}, 3 \mathrm{H})$, $6.88(\mathrm{~d}, J=8.6 \mathrm{~Hz}, 2 \mathrm{H}), 6.76(\mathrm{~s}, 1 \mathrm{H}), 5.33(\mathrm{dd}, J=6.6,5.3 \mathrm{~Hz}, 1 \mathrm{H}), 3.66-3.51(\mathrm{~m}, 2 \mathrm{H}), 2.28$ $-2.23(\mathrm{~m}, 2 \mathrm{H}) \mathrm{ppm}$.

${ }^{13} \mathrm{C} \mathrm{NMR}\left(126 \mathrm{MHz}, \mathrm{CDCl}_{3}\right) \delta 158.7(\mathrm{~d}, J=1.5 \mathrm{~Hz}), 156.0(\mathrm{q}, J=36.9 \mathrm{~Hz}), 138.4,128.1,127.4$, $126.0(\mathrm{q}, J=3.8 \mathrm{~Hz}), 124.5,123.2(\mathrm{q}, J=271.63 \mathrm{~Hz}), 122.6(\mathrm{q}, J=32.8 \mathrm{~Hz}), 114.9(\mathrm{q}, J=$ $287.12 \mathrm{~Hz}), 114.6,78.3,36.2,36.0 \mathrm{ppm}$.

${ }^{19} \mathrm{~F}$ NMR $\left(377 \mathrm{MHz}, \mathrm{CDCl}_{3}\right) \delta-61.71,-76.01 \mathrm{ppm}$.

HRMS (ESI) m/z: [M+Na]+ Calcd for C18H15F6NO2Na 414.0899; Found 414.0895. 
$N$-(3-(4-chlorophenoxy)-3-phenylpropyl)-2,2,2-trifluoroacetamide (28)<smiles>N=CNCCC(Oc1ccc(Cl)cc1)c1ccccc1</smiles>

28

Reaction conducted using Procedure I with 4-chlorophenol (0.4 mmol, $51.42 \mathrm{mg}, 2$ equiv) Following workup, the obtained residue was purified by column chromatography on silica gel eluting with a solvent mixture of ethyl acetate:hexanes (08:92 (v:v)) to afford $35.1 \mathrm{mg}$ of $\mathbf{2 8}$ as a light-yellow oil (49\% yield).

NMR Spectroscopy:

${ }^{1} \mathrm{H}$ NMR $\left(500 \mathrm{MHz}, \mathrm{CDCl}_{3}\right) \delta 7.38-7.33(\mathrm{~m}, 2 \mathrm{H}), 7.32-7.28(\mathrm{~m}, 3 \mathrm{H}), 7.16-7.11(\mathrm{~m}, 2 \mathrm{H}), 6.75$ - $6.71(\mathrm{~m}, 2 \mathrm{H}), 5.24(\mathrm{t}, J=6.0 \mathrm{~Hz}, 1 \mathrm{H}), 3.65-3.48(\mathrm{~m}, 2 \mathrm{H}), 2.21(\mathrm{q}, J=6.5 \mathrm{~Hz}, 2 \mathrm{H}) \mathrm{ppm}$.

${ }^{13} \mathrm{C}$ NMR $\left(126 \mathrm{MHz}, \mathrm{CDCl}_{3}\right) \delta 160.0(\mathrm{q}, J=36.8 \mathrm{~Hz}), 154.7,138.7,128.4,128.0,127.3,125.4$, 124.6, 114.8 (q, $J=288.17 \mathrm{~Hz}), 116.0,78.6,36.3,35.9$. (q, $J=271.63 \mathrm{~Hz}), 122.6(\mathrm{q}, J=32.8$ $\mathrm{Hz}), 114$. (q, $J=287.12 \mathrm{~Hz}), 114.6,78.3,36.2,36.0 \mathrm{ppm}$.

${ }^{19} \mathrm{~F}$ NMR $\left(377 \mathrm{MHz}, \mathrm{CDCl}_{3}\right) \delta-76.02 \mathrm{ppm}$.

HRMS (ESI) m/z: [M+Na]+ Calcd for C17H15ClF3NO2Na 380.0636; Found 380.0632.

2,2,2-trifluoro- $N$-(3-phenyl-3-((4-(trifluoromethyl)phenyl)thio)propyl)acetamide (29)<smiles>FC(F)[In]CCC(Sc1ccc(C(F)(F)F)cc1)c1ccccc1</smiles>

29

Reaction conducted using Procedure I with 4-trifluoromethylbenzenethiol (0.4 mmol, $71.27 \mathrm{mg}$ 2 equiv). Following workup, the obtained residue was purified by column chromatography on silica gel eluting with a solvent mixture of ethyl acetate:hexanes (10:90 (v:v)) to afford $53.77 \mathrm{mg}$ of 29 as a white solid (66\% yield).

NMR Spectroscopy:

${ }^{1} \mathrm{H}$ NMR $\left(500 \mathrm{MHz}, \mathrm{CDCl}_{3}\right) \delta 7.43(\mathrm{~d}, J=8.1 \mathrm{~Hz}, 2 \mathrm{H}), 7.33-7.22(\mathrm{~m}, 7 \mathrm{H}), 6.24(\mathrm{~s}, 1 \mathrm{H}), 4.24$ $(\mathrm{dd}, J=8.4,6.5 \mathrm{~Hz}, 1 \mathrm{H}), 3.48(\mathrm{dq}, J=13.5,6.7 \mathrm{~Hz}, 1 \mathrm{H}), 3.35(\mathrm{dq}, J=13.4,6.6 \mathrm{~Hz}, 1 \mathrm{H}) \mathrm{ppm}$. ${ }^{13} \mathrm{C} \mathrm{NMR}\left(126 \mathrm{MHz}, \mathrm{CDCl}_{3}\right) \delta 157.3(\mathrm{q}, J=37.0 \mathrm{~Hz}), 140.3,139.5(\mathrm{~d}, J=1.4 \mathrm{~Hz}), 131.3,129.2$ $(\mathrm{q}, J=32.73 \mathrm{~Hz}), 129.2,128.3,127.7,125.8(\mathrm{q}, J=3.7 \mathrm{~Hz}), 124.1(\mathrm{q}, J=272.08 \mathrm{~Hz}) \mathrm{ppm}$.

${ }^{19} \mathrm{~F}$ NMR $\left(377 \mathrm{MHz}, \mathrm{CDCl}_{3}\right) \delta-62.65,-75.99 \mathrm{ppm}$.

HRMS (ESI) m/z: [M+Na]+ Calcd for C18H15F6NOS Na 430.0670; Found 430.0668. 
$N$-(3-((4-chlorophenyl)thio)-3-phenylpropyl)-2,2,2-trifluoroacetamide (30)<smiles></smiles>

30

Reaction conducted using Procedure I with p-Chlorothiophenol ( $0.4 \mathrm{mmol}, 57.85 \mathrm{mg} 2$ equiv). Following workup, the obtained residue was purified by column chromatography on silica gel eluting with a solvent mixture of ethyl acetate:hexanes (10:90 (v:v)) to afford $71.02 \mathrm{mg}$ of $\mathbf{3 0}$ as a white solid ( $95 \%$ yield).

NMR Spectroscopy:

${ }^{1} \mathrm{H}$ NMR $\left(500 \mathrm{MHz}, \mathrm{CDCl}_{3}\right) \delta 7.30-7.22(\mathrm{~m}, 3 \mathrm{H}), 7.20-7.11(\mathrm{~m}, 6 \mathrm{H}), 6.23(\mathrm{~s}, 1 \mathrm{H}), 4.10(\mathrm{dd}, J$ $=8.3,6.7 \mathrm{~Hz}, 1 \mathrm{H}), 3.47(\mathrm{dq}, J=13.4,6.7 \mathrm{~Hz}, 1 \mathrm{H}), 3.35(\mathrm{dq}, J=13.4,6.6 \mathrm{~Hz}, 1 \mathrm{H}), 2.26-2.14$ $(\mathrm{m}, 2 \mathrm{H}) \mathrm{ppm}$.

${ }^{13} \mathrm{C}$ NMR (126 MHz, CDCl3) $\delta 157.1$ (q, J = 37.0 Hz), 140.4, 134.5, 134.1, 132.1, 129.1, 128.9, $127.9,127.6,115.7(\mathrm{q}, \mathrm{J}=287.9 \mathrm{~Hz}), 51.9,38.5,35.0 \mathrm{ppm}$.

${ }^{19} \mathrm{~F}$ NMR $\left(377 \mathrm{MHz}, \mathrm{CDCl}_{3}\right) \delta-75.98 \mathrm{ppm}$.

HRMS (ESI) m/z: [M+NH4]+ Calcd for C17H19ClF3N2OS 391.0850; Found 391.0850. 
(4-methoxyphenyl)(5-nitro-2-(1-((4-(trifluoromethyl)phenyl)thio)butyl)benzofuran-3yl)methanone (31)<smiles>CCCC(Sc1ccc(C(F)(F)F)cc1)c1oc2ccc([N+](=O)[O-])cc2c1C(=O)c1ccc(OC)cc1</smiles>

31

Reaction conducted using Procedure I with 4-trifluoromethylbenzenethiol (0.4 mmol, $71.27 \mathrm{mg}$ 2 equiv),Following workup, the obtained residue was purified by column chromatography on silica gel eluting with a solvent mixture of ethyl acetate:hexanes (07:93 (v:v)) to afford $94.2 \mathrm{mg}$ of $\mathbf{3 1}$ as a clear glassy solid ( $89 \%$ yield).

NMR Spectroscopy:

${ }^{1} \mathrm{H}$ NMR $\left(\left(500 \mathrm{MHz}, \mathrm{CDCl}_{3}\right) \delta 8.24(\mathrm{dd}, J=9.1,2.4 \mathrm{~Hz}, 1 \mathrm{H}), 8.05(\mathrm{~d}, J=2.3 \mathrm{~Hz}, 1 \mathrm{H}), 7.62(\mathrm{~d}, J\right.$ $=9.0 \mathrm{~Hz}, 1 \mathrm{H}), 7.48(\mathrm{~d}, J=8.4 \mathrm{~Hz}, 2 \mathrm{H}), 7.32(\mathrm{~s}, 1 \mathrm{H}), 7.23(\mathrm{~d}, J=8.2 \mathrm{~Hz}, 2 \mathrm{H}), 6.89-6.86(\mathrm{~m}$, $2 \mathrm{H}), 5.01(\mathrm{dd}, J=8.9,6.5 \mathrm{~Hz}, 1 \mathrm{H}), 3.89(\mathrm{~s}, 3 \mathrm{H}), 2.24-2.16(\mathrm{~m}, 1 \mathrm{H}), 2.12-2.03(\mathrm{~m}, 1 \mathrm{H}), 1.66-$ $1.53(\mathrm{~m}, 1 \mathrm{H}), 1.47-1.38(\mathrm{~m}, 1 \mathrm{H}), 1.00(\mathrm{t}, J=7.3 \mathrm{~Hz}, 3 \mathrm{H}) \mathrm{ppm}$.

${ }^{13} \mathrm{C}$ NMR $\left(126 \mathrm{MHz}, \mathrm{CDCl}_{3}\right) \delta 188.3,164.5,164.1,156.3,144.9,138.5(\mathrm{~d}, J=1.5 \mathrm{~Hz}), 132.2$, $131.8,130.2,129.7(\mathrm{q}, J=32.7 \mathrm{~Hz}), 127.1,124.9,122.8,120.6(\mathrm{q}, \mathrm{J}=272.29 \mathrm{~Hz}), 126.9$, $125.9,125.9,125.8(\mathrm{q}, J=3.7 \mathrm{~Hz}), 121.1,119.3,118.1,114.2,112.1,55.7,44.4,35.0,21.1$, $13.8 \mathrm{ppm}$.

${ }^{19} \mathrm{~F}$ NMR $\left(377 \mathrm{MHz}, \mathrm{CDCl}_{3}\right) \delta-62.67 \mathrm{ppm}$.

HRMS (ESI) m/z: [M+H]+ Calcd for C27H23F3NO5S 530.1244; Found 530.1241. 
<smiles>CCCC(Sc1ccc(Cl)cc1)c1oc2ccc([N+](=O)[O-])cc2c1C(=O)c1ccc(OC)cc1</smiles>

32

Reaction conducted using Procedure I with p-Chlorothiophenol ( $0.4 \mathrm{mmol}, 57.85 \mathrm{mg} 2$ equiv). ,Following workup, the obtained residue was purified by column chromatography on silica gel eluting with a solvent mixture of ethyl acetate:hexanes $(07: 93(\mathrm{v}: \mathrm{v}))$ followed by a second column eluting with solvent mixture of dichloromethane/cyclohexane (60:40 (v:v)) to afford $67.45 \mathrm{mg} 32$ as a clear glassy solid (68\% yield).

NMR Spectroscopy:

${ }^{1} \mathrm{H}$ NMR $\left.500 \mathrm{MHz}, \mathrm{CDCl} 3\right) \delta 8.24(\mathrm{dd}, \mathrm{J}=9.1,2.4 \mathrm{~Hz}, 1 \mathrm{H}), 8.06(\mathrm{~d}, \mathrm{~J}=2.3 \mathrm{~Hz}, 1 \mathrm{H}), 7.62(\mathrm{~d}, \mathrm{~J}=$ $9.1 \mathrm{~Hz}, 1 \mathrm{H}), 7.44(\mathrm{~d}, \mathrm{~J}=8.5 \mathrm{~Hz}, 2 \mathrm{H}), 7.14-7.10(\mathrm{~m}, 2 \mathrm{H}), 6.96-6.93(\mathrm{~m}, 2 \mathrm{H}), 6.92-6.88$ (m, 2H), $4.84(\mathrm{dd}, \mathrm{J}=8.9,6.4 \mathrm{~Hz}, 1 \mathrm{H}), 3.91(\mathrm{~s}, 3 \mathrm{H}), 2.21(\mathrm{dtd}, \mathrm{J}=13.6,9.3,5.7 \mathrm{~Hz}, 1 \mathrm{H}), 2.09$ (ddt, J = 13.6, 9.6, 6.2 Hz, 1H), $1.63-1.53(\mathrm{~m}, 1 \mathrm{H}), 1.49-1.39(\mathrm{~m}, 1 \mathrm{H}), 0.99$ (t, J = 7.4 Hz, $3 \mathrm{H}) \mathrm{ppm}$.

${ }^{13} \mathrm{C}$ NMR $\left(126 \mathrm{MHz}, \mathrm{CDCl}_{3}\right) \delta 188.1,164.3,164.3,156.3,144.8,135.1,134.7,131.8,131.5$, $130.1,129.3,127.1,121.0,119.4,118.0,114.1,112.0,55.7,45.4,34.7,21.1,13.8 \mathrm{ppm}$.

HRMS (ESI) m/z: [M+H]+ Calcd for C26H23CINO5S 496.0980; Found 496.0981. 
(2-(1-(4-(5-fluorobenzo[d]isoxazol-3-yl)piperidin-1-yl)butyl)-5-nitrobenzofuran-3-yl)(4methoxyphenyl)methanone (33)

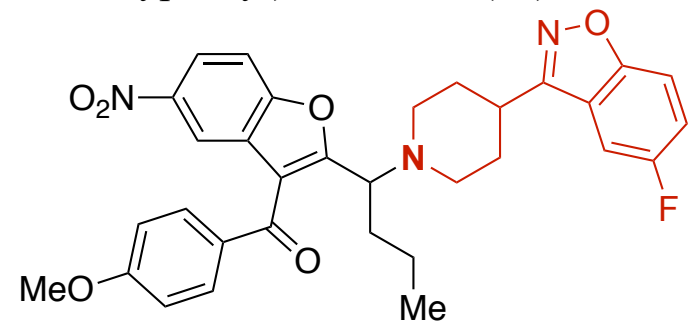

33

Reaction conducted using Procedure $\mathbf{J}$ 5-fluoro-3-(4-piperidinyl)-1,2-benzisoxazole ( $0.4 \mathrm{mmol}$, $88.1 \mathrm{mg} 2$ equiv). ,Following workup, the obtained residue was purified by column chromatography on silica gel eluting with a solvent mixture of ethyl acetate: hexanes (10:90 (v:v)) to afford $54.87 \mathrm{mg}$ of $\mathbf{3 3}$ as a clear glassy solid ( $48 \%$ yield).

NMR Spectroscopy:

${ }^{1} \mathrm{H}$ NMR $(500 \mathrm{MHz}, \mathrm{CDCl} 3) \delta 8.30(\mathrm{~d}, \mathrm{~J}=2.3 \mathrm{~Hz}, 1 \mathrm{H}), 8.27(\mathrm{dd}, \mathrm{J}=9.0,2.3 \mathrm{~Hz}, 1 \mathrm{H}), 7.87$ (d, J $=8.8 \mathrm{~Hz}, 2 \mathrm{H}), 7.65(\mathrm{~d}, \mathrm{~J}=9.0 \mathrm{~Hz}, 1 \mathrm{H}), 7.58(\mathrm{dd}, \mathrm{J}=8.7,5.1 \mathrm{~Hz}, 1 \mathrm{H}), 7.21(\mathrm{dd}, \mathrm{J}=8.5,2.1$ $\mathrm{Hz}, 1 \mathrm{H}), 7.00(\mathrm{dd}, \mathrm{J}=9.7,2.6 \mathrm{~Hz}, 3 \mathrm{H}), 4.17(\mathrm{dd}, \mathrm{J}=8.5,6.7 \mathrm{~Hz}, 1 \mathrm{H}), 3.92(\mathrm{~s}, 3 \mathrm{H}), 3.22(\mathrm{dd}$, $\mathrm{J}=11.5,4.7 \mathrm{~Hz}, 1 \mathrm{H}), 2.91(\mathrm{tt}, \mathrm{J}=7.0,3.8 \mathrm{~Hz}, 2 \mathrm{H}), 2.30(\mathrm{td}, \mathrm{J}=11.1,3.3 \mathrm{~Hz}, 1 \mathrm{H}), 2.21(\mathrm{td}, \mathrm{J}$ $=11.4,2.8 \mathrm{~Hz}, 1 \mathrm{H}), 2.10-1.90(\mathrm{~m}, 6 \mathrm{H}), 1.43(\mathrm{tdd}, \mathrm{J}=9.4,7.6,6.1 \mathrm{~Hz}, 1 \mathrm{H}), 1.37-1.26(\mathrm{~m}$, $1 \mathrm{H}), 0.95(\mathrm{t}, \mathrm{J}=7.3 \mathrm{~Hz}, 3 \mathrm{H}) \mathrm{ppm}$.

${ }^{13} \mathrm{C}$ NMR $\left(126 \mathrm{MHz}, \mathrm{CDCl}_{3}\right) \delta 189.1,164.5,164.2(\mathrm{~d}, J=250.75 \mathrm{~Hz}), 164.4,164.0(\mathrm{~d}, J=13.6$ $\mathrm{Hz}), 163.2,156.4,144.9,132.1,131.0,127.6,122.6,122.5,120.8,118.1,117.4(\mathrm{~d}, J=1.3 \mathrm{~Hz})$, $114.4,112.5(\mathrm{~d}, J=25.3 \mathrm{~Hz}), 112.1,97.6(\mathrm{~d}, J=26.7 \mathrm{~Hz}), 60.7,55.8,52.1,48.4,34.7,32.3$, $31.1,31.0,19.9,14.1 \mathrm{ppm}$.

${ }^{19} \mathrm{~F}$ NMR (377 MHz, CDCl3) $\delta-109.61 \mathrm{ppm}$.

HRMS (ESI) m/z: [M+H]+ Calcd for C32H31FN3O6 572.2191; Found 572.2185. 
(4-methoxyphenyl)(5-nitro-2-(1-(4-(pyridin-2-yl)piperazin-1-yl)butyl)benzofuran-3yl)methanone (34)<smiles>CCCC(c1oc2ccc([N+](=O)[O-])cc2c1C(=O)c1ccc(OC)cc1)N1CCN(c2ccccn2)CC1</smiles>

Reaction conducted using Procedure $\mathbf{J}$ with 1-(2-pyridyl)piperazine $(0.4 \mathrm{mmol}, 65.29 .1 \mathrm{mg} 2$ equiv). ,Following workup, the obtained residue was purified by column chromatography on silica gel eluting with a solvent mixture of DCM: Diethylether (40:60 (v:v)) to afford $48.37 \mathrm{mg}$ of $\mathbf{3 4}$ as a light yellow oil ( $47 \%$ yield).

NMR Spectroscopy:

${ }^{1} \mathrm{H}$ NMR (500 MHz, CDCl3) $\delta 8.24$ (dd, J = 9.0, $\left.2.4 \mathrm{~Hz}, 1 \mathrm{H}\right), 8.21(\mathrm{~d}, \mathrm{~J}=2.3 \mathrm{~Hz}, 1 \mathrm{H}), 8.13$ (ddd, $\mathrm{J}=4.9,2.1,0.9 \mathrm{~Hz}, 1 \mathrm{H}), 7.86-7.82(\mathrm{~m}, 2 \mathrm{H}), 7.62(\mathrm{~d}, \mathrm{~J}=9.0 \mathrm{~Hz}, 1 \mathrm{H}), 7.42(\mathrm{ddd}, \mathrm{J}=8.9,7.1$, $2.0 \mathrm{~Hz}, 1 \mathrm{H}), 7.00-6.96(\mathrm{~m}, 2 \mathrm{H}), 6.63-6.52(\mathrm{~m}, 2 \mathrm{H}), 4.24(\mathrm{dd}, \mathrm{J}=8.9,6.3 \mathrm{~Hz}, 1 \mathrm{H}), 3.92(\mathrm{~s}$, $3 \mathrm{H}$ ), 3.54 (ddd, J = 12.3, 7.0, $3.2 \mathrm{~Hz}, 2 \mathrm{H}$ ), 3.42 (ddd, $\mathrm{J}=12.4,7.0,3.3 \mathrm{~Hz}, 2 \mathrm{H}$ ), 2.70 (ddd, J = $10.6,6.9,3.2 \mathrm{~Hz}, 2 \mathrm{H}), 2.58$ (ddd, J = 10.8, 7.0, $3.4 \mathrm{~Hz}, 2 \mathrm{H}), 2.14-1.93(\mathrm{~m}, 2 \mathrm{H}), 1.50-1.35$ $(\mathrm{m}, 1 \mathrm{H}), 1.36-1.26(\mathrm{~m}, 1 \mathrm{H}), 0.95(\mathrm{t}, \mathrm{J}=7.3 \mathrm{~Hz}, 3 \mathrm{H}) \mathrm{ppm}$.

${ }^{13} \mathrm{C}$ NMR $\left(126 \mathrm{MHz}, \mathrm{CDCl}_{3}\right) \delta 188.9,164.4,164.3,159.5,156.4,148.1,144.8,137.6,132.0$, $130.9,127.4,120.7,120.6,118.1,114.4,113.5,112.1,107.1,60.4,55.8,49.8,45.7,32.2,19.8$, $14.1 \mathrm{ppm}$.

HRMS (ESI) m/z: [M+H]+ Calcd for C29H31N4O5 515.2289; Found 515.2290. 


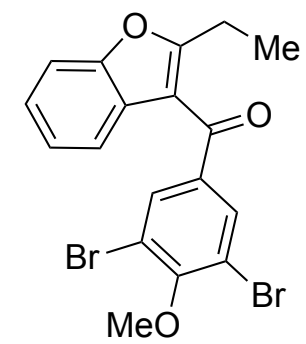

Compound 36:

Compound 36 was prepared following the literature procedure ${ }^{3}$ To a $25 \mathrm{~mL}$ round-bottom flask containing a magnetic stir bar was added benzobromarone $(842.2 \mathrm{mg}, 2.0 \mathrm{mmol})$. The flask was subsequently charged with a mixture of acetonitrile and methanol $(9: 1,8 \mathrm{~mL})$, followed by diisopropylethylamine $(0.72 \mathrm{~mL}, 0.75$ equiv) and stirred for $10 \mathrm{~min}$. To the stirring reaction mixture, trimethylsilyldizaomethane $(2.0 \mathrm{M}$ Hexane solution, $1.5 \mathrm{~mL}, 0.75$ equiv) was added dropwise. A funnel was placed upside down on top of the flask to ensure minimal solvent evaporation. The reaction was allowed to stir at room temperature overnight. The reaction was concentrated under vacuum and the reside was purified by column chromatography eluting with a solvent mixture pentane:ethylacetate (80:20 (v:v)) to afford $.753 .7 \mathrm{mg}$ of 36 (86\% yield.) Spectra data matched those in the literature. ${ }^{3}$<smiles>CC(C)NCCc1ccccc1</smiles>

\section{Compound 37}

To a flame-dried $100 \mathrm{~mL}$ round-bottom flask containing magnetic stir bar at $0{ }^{\circ} \mathrm{C}$ under nitrogen was added phenylethylamine ( $2 \mathrm{~g}, 0.016 \mathrm{~mol}, 1$ equiv), anhydrous dichloromethane $(40 \mathrm{~mL}, 0.4 \mathrm{M}$ to substrate), and pyridine ( $3.9 \mathrm{~g}, 4.0 \mathrm{~mL}, 3$ equiv) followed by dropwise addition of trifluoroacetic anhydride $(6.9 \mathrm{~g}, 4.6 \mathrm{~mL}, 2$ equiv). After stirring at room temperature overnight, the reaction was slowly quenched with water, then extracted with dichloromethane $(2 \mathrm{x})$. The organic extracts were combined and washed with $\mathrm{H}_{2} \mathrm{O}(2 \times 100 \mathrm{~mL})$ then brine $(1 \times 100 \mathrm{~mL})$. The organic layer was dried (anhydrous $\mathrm{Na}_{2} \mathrm{SO}_{4}$ ), filtered and concentrated under vacuum. Purification of the desired compound was performed by flash chromatography. a solvent mixture pentane:ethylacetate (90:10 (v:v)) to afford $3.1 \mathrm{~g}(90 \%$ yield) of the product as a white solid. Spectra data matched those in the literature. ${ }^{23}$ 
<smiles>CC(C)NCCCc1ccccc1</smiles>

To a flame-dried $100 \mathrm{~mL}$ round-bottom flask containing magnetic stir bar at $0{ }^{\circ} \mathrm{C}$ under nitrogen was added 3 phenyl propylamine ( $2 \mathrm{~g}, 0.015 \mathrm{~mol}, 1$ equiv), anhydrous dichloromethane (40 $\mathrm{mL}$, $0.4 \mathrm{M}$ to substrate), and pyridine $(3.5 \mathrm{~g}, 3.6 \mathrm{~mL}, 3$ equiv) followed by dropwise addition of trifluoroacetic anhydride $(6.21 \mathrm{~g}, 4.1 \mathrm{~mL}, 2$ equiv). After stirring at room temperature overnight, the reaction was slowly quenched with water, then extracted with dichloromethane $(2 \mathrm{x})$. The organic extracts were combined and washed with $\mathrm{H}_{2} \mathrm{O}(2 \times 100 \mathrm{~mL})$ then brine $(1 \mathrm{x} 100 \mathrm{~mL})$. The organic layer was dried (anhydrous $\mathrm{Na}_{2} \mathrm{SO}_{4}$ ), filtered and concentrated under vacuum. Purification was of the desired compound was performed by flash chromatography. a solvent mixture pentane: ethylacetate $(90: 10(\mathrm{v}: \mathrm{v}))$ to afford $3.2 \mathrm{~g}(92 \%$ yield $)$ of the product as a light-yellow solid. Spectra data matched those in the literature. ${ }^{23}$

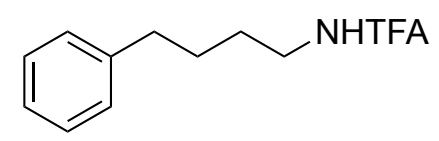

To a flame-dried $100 \mathrm{~mL}$ round-bottom flask containing magnetic stir bar at $0{ }^{\circ} \mathrm{C}$ under nitrogen was added 3 butyl propylamine ( $2 \mathrm{~g}, 0.013 \mathrm{~mol}, 1$ equiv), anhydrous dichloromethane ( $33 \mathrm{~mL}$, $0.4 \mathrm{M}$ to substrate), and pyridine $(3.1 \mathrm{~g}, 3.25 \mathrm{~mL}, 3$ equiv) followed by dropwise addition of trifluoroacetic anhydride $(5.6 \mathrm{~g}, 3.7 \mathrm{~mL}, 2$ equiv). After stirring at room temperature overnight, the reaction was slowly quenched with water, then extracted with dichloromethane $(2 \mathrm{x})$. The organic extracts were combined and washed with $\mathrm{H}_{2} \mathrm{O}(2 \times 100 \mathrm{~mL})$ then brine $(1 \mathrm{x} 100 \mathrm{~mL})$. The organic layer was dried (anhydrous $\mathrm{Na}_{2} \mathrm{SO}_{4}$ ), filtered and concentrated under vacuum. Purification was of the desired compound was performed by flash chromatography. a solvent mixture pentane:ethylacetate (90:10 (v:v)) to afford $2.86 \mathrm{~g}$ (90\% yield) of the product as a light-yellow solid. Spectra data matched those in the literature. ${ }^{24}$ 


\section{References}

(1) Hofstra, J. L.; DeLano, T. J.; Reisman, S. E. Synthesis of Chiral Bis(oxazoline) Ligands: (3aR,3a'R,8aS,8a'S)-2,2'-(Cyclopropane-1,1-Diyl)Bis(3a,8a-Dihydro-8H-Indeno[1,2d]Oxazole). Org. Synth. 2020, 97, 172-188.

(2) Tanaka, J.; Suib, S. L. Experimental Methods in Inorganic Chemistry; Prentice-Hall, Inc., 1999.

(3) Hu, H.; Chen, S.-J.; Mandal, M.; Pratik, S. M.; Buss, J. A.; Krska, S. W.; Cramer, C. J.; Stahl, S. S. Copper-Catalysed Benzylic C-H Coupling with Alcohols via Radical Relay Enabled by Redox Buffering. Nat. Catal. 2020, 3, 358-367.

(4) Clark, J. R.; Feng, K.; Sookezian, A.; White, M. C. Manganese-Catalysed Benzylic C(sp ${ }^{3}$ H Amination for Late-Stage Functionalization. Nat. Chem. 2018, 10, 583-591.

(5) Ozawa, J.; Kanai, M. Silver-Catalyzed C $\left(\mathrm{sp}^{3}\right)-$ H Chlorination. Org. Lett. 2017, 19, 14301433.

(6) Pathi, S. L.; Kankan, R. N.; Puppala, R. A Process of Preparing Imatinib. 2008MU02726, 2008.

(7) Combe, S. H.; Hosseini, A.; Parra, A.; Schreiner, P. R. Mild Aliphatic and Benzylic Hydrocarbon $\mathrm{C}-\mathrm{H}$ Bond Chlorination Using Trichloroisocyanuric Acid. J. Org. Chem. 2017, 82, 2407-2413.

(8) Han, L.; Xia, J.-B.; You, L.; Chen, C. Ketone-Catalyzed Photochemical C( $\left.\mathrm{sp}^{3}\right)-\mathrm{H}$ Chlorination. Tetrahedron 2017, 73, 3696-3701.

(9) Kharasch, M. S.; Brown, H. C. Chlorinations with Sulfuryl Chloride. I. The PeroxideCatalyzed Chlorination of Hydrocarbons. J. Am. Chem. Soc. 1939, 61, 2142-2150.

(10) Liu, W.; Groves, J. T. Manganese Porphyrins Catalyze Selective C-H Bond Halogenations. J. Am. Chem. Soc. 2010, 132, 12847-12849.

(11) Takeda, M.; Shintani, R.; Hayashi, T. Enantioselective Synthesis of $\alpha$-Tri- and $\alpha$ Tetrasubstituted Allylsilanes by Copper-Catalyzed Asymmetric Allylic Substitution of Allyl Phosphates with Silylboronates. J. Org. Chem. 2013, 78, 5007-5017.

(12) Li, C.; Wang, Q.; Zhang, J.-Q.; Ye, J.; Xie, J.; Xu, Q.; Han, L.-B. Water Determines the Products: An Unexpected Brønsted Acid-Catalyzed PO-R Cleavage of P(III) Esters Selectively Producing P(O)-H and P(O)-R Compounds. Green Chem. 2019, 21, 29162922.

(13) Stach, T.; Dräger, J.; Huy, P. H. Nucleophilic Substitutions of Alcohols in High Levels of Catalytic Efficiency. Org. Lett. 2018, 20, 2980-2983.

(14) Iwamoto, H.; Endo, K.; Ozawa, Y.; Watanabe, Y.; Kubota, K.; Imamoto, T.; Ito, H. Copper(I)-Catalyzed Enantioconvergent Borylation of Racemic Benzyl Chlorides Enabled by Quadrant-by-Quadrant Structure Modification of Chiral Bisphosphine Ligands. Angew. Chem. Int. Ed. 2019, 58, 11112-11117.

(15) Lee, C.-H.; Lee, S.-M.; Min, B.-H.; Kim, D.-S.; Jun, C.-H. Ferric(III) Chloride Catalyzed Halogenation Reaction of Alcohols and Carboxylic Acids Using $\alpha, \alpha-$ Dichlorodiphenylmethane. Org. Lett. 2018, 20, 2468-2471.

(16) Liang, S.; Hammond, G. B.; Xu, B. Metal-Free Regioselective Hydrochlorination of Unactivated Alkenes via a Combined Acid Catalytic System. Green Chem. 2018, 20, 680684.

(17) Tandiary, M. A.; Masui, Y.; Onaka, M. Chlorination of Benzylic and Allylic Alcohols with Trimethylsilyl Chloride Enhanced by Natural Sodium Montmorillonite. Synlett 2014, 25 , 2639-2643. 
(18) Ayala, C. E.; Villalpando, A.; Nguyen, A. L.; McCandless, G. T.; Kartika, R. Chlorination of Aliphatic Primary Alcohols via Triphosgene-Triethylamine Activation. Org. Lett.

2012, 14, 3676-3679.

(19) Xiang, M.; Zhou, C.; Yang, X.-L.; Chen, B.; Tung, C.-H.; Wu, L.-Z. Visible LightCatalyzed Benzylic $\mathrm{C}-\mathrm{H}$ Bond Chlorination by a Combination of Organic Dye (Acr $\left.{ }^{+}-\mathrm{Mes}\right)$ and $\mathrm{N}$-Chlorosuccinimide. J. Org. Chem. 2020, 85, 9080-9087.

(20) Wang, Y.; Li, G.-X.; Yang, G.; He, G.; Chen, G. A Visible-Light-Promoted Radical Reaction System for Azidation and Halogenation of Tertiary Aliphatic C-H Bonds. Chem. Sci. 2016, 7, 2679-2683.

(21) Sasmal, S.; Rana, S.; Lahiri, G. K.; Maiti, D. Manganese-Salen Catalyzed Oxidative Benzylic Chlorination. J. Chem. Sci. 2018, 130, 88.

(22) Chiba, S.; Zhang, L.; Ang, G. Y.; Hui, B. W.-Q. Generation of Iminyl Copper Species from $\alpha$-Azido Carbonyl Compounds and Their Catalytic $\mathrm{C}-\mathrm{C}$ Bond Cleavage under an Oxygen Atmosphere. Org. Lett. 2010, 12, 2052-2055.

(23) Piszel, P. E.; Vasilopoulos, A.; Stahl, S. S. Oxidative Amide Coupling from Functionally Diverse Alcohols and Amines Using Aerobic Copper/Nitroxyl Catalysis. Angew. Chem. Int. Ed. 2019, 58, 12211-12215.

(24) Hu, Q.-P.; Cheng, J.; Wang, Y.; Shi, J.; Wang, B.-Q.; Hu, P.; Zhao, K.-Q.; Pan, F. Remote Regioselective Radical C-H Functionalization of Unactivated C-H Bonds in Amides: The Synthesis of Gem -Difluoroalkenes. Org. Lett. 2021, 23, 4457-4462. 


\section{NMR Spectra}

${ }^{1} \mathbf{H}$ NMR $\left(500 \mathrm{MHz}, \mathrm{CDCl}_{3}\right)$ of 2-B

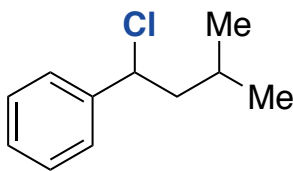

2-B

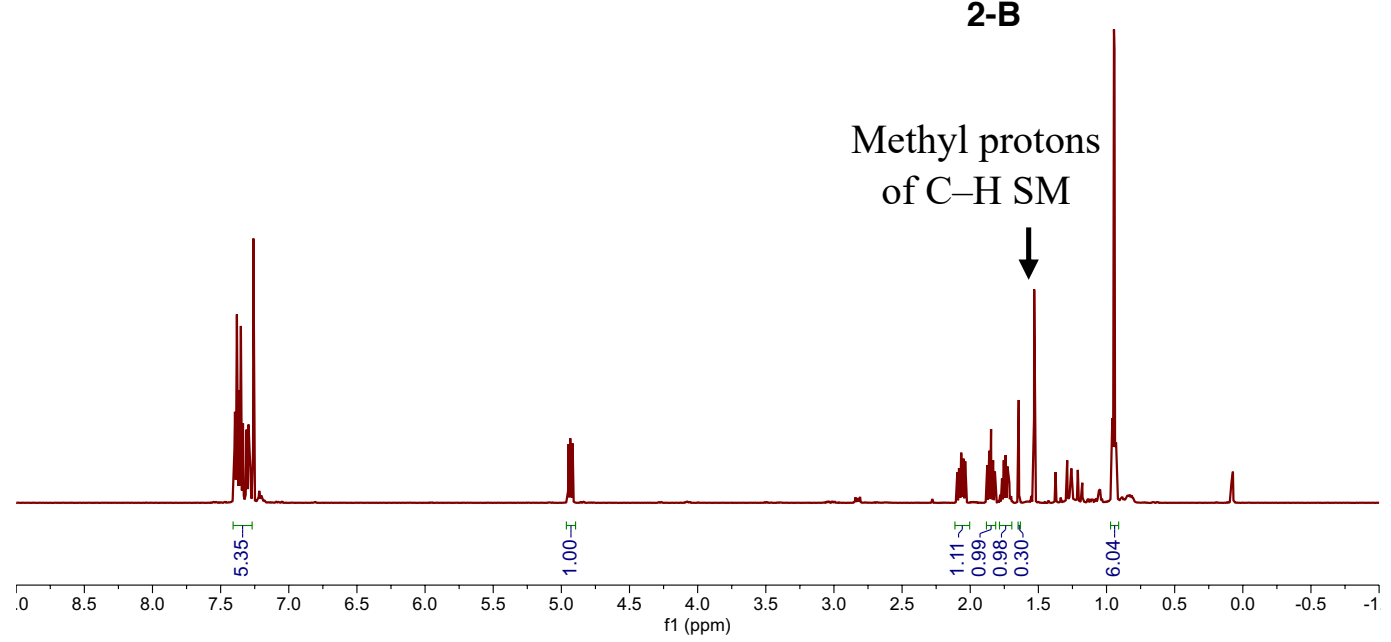

${ }^{13} \mathbf{C ~ N M R}\left(126 \mathrm{MHz}, \mathrm{CDCl}_{3}\right)$ of 2-B

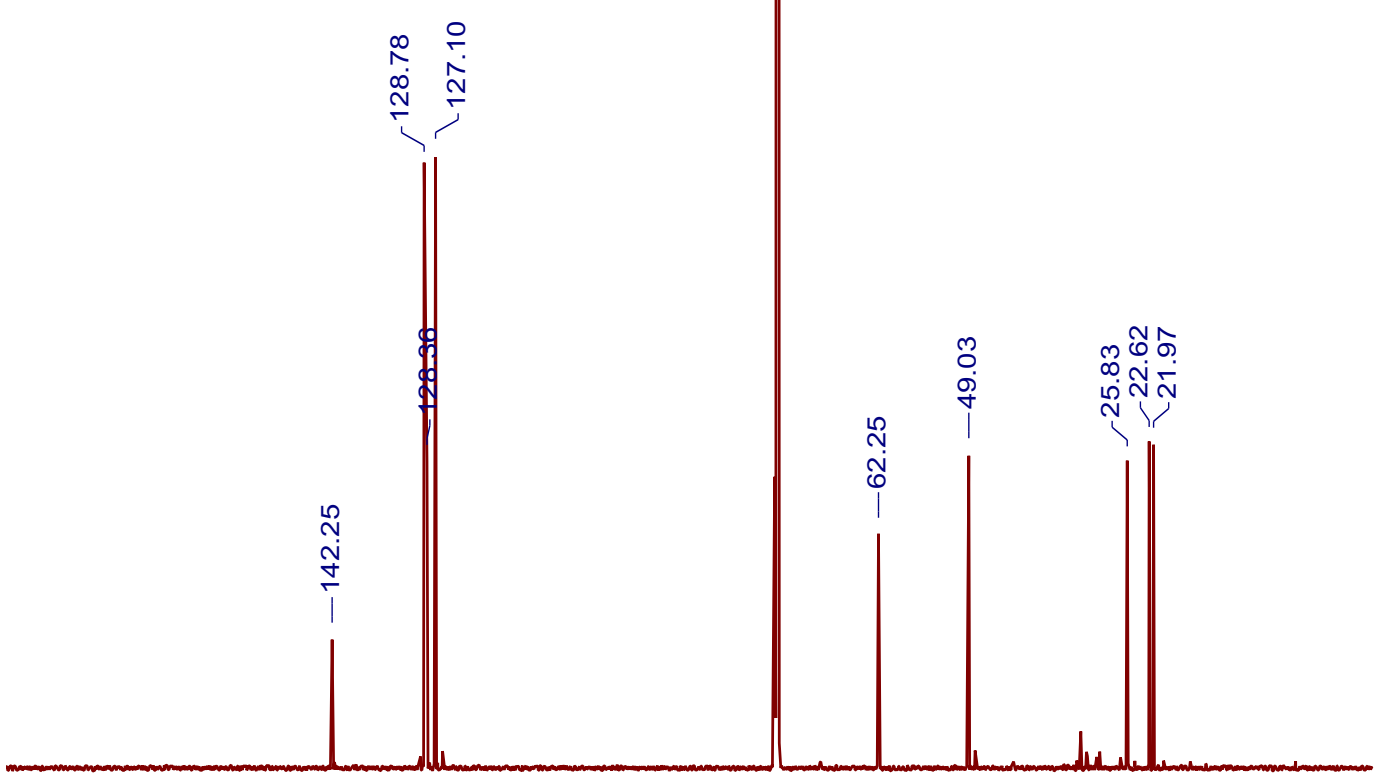

\begin{tabular}{|llllllllllllllllllllllllll}
\hline 0 & 180 & 170 & 160 & 150 & 140 & 130 & 120 & 110 & 100 & 90 & 80 & 70 & 60 & 50 & 40 & 30 & 20 & 10 & 0 & -1
\end{tabular} 
${ }^{1} \mathbf{H}$ NMR $\left(400 \mathrm{MHz}, \mathrm{CDCl}_{3}\right)$ of $\mathbf{6}$

궁ำกำ

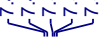

든 뉸윤은

$\sqrt[3]{\log \log }$
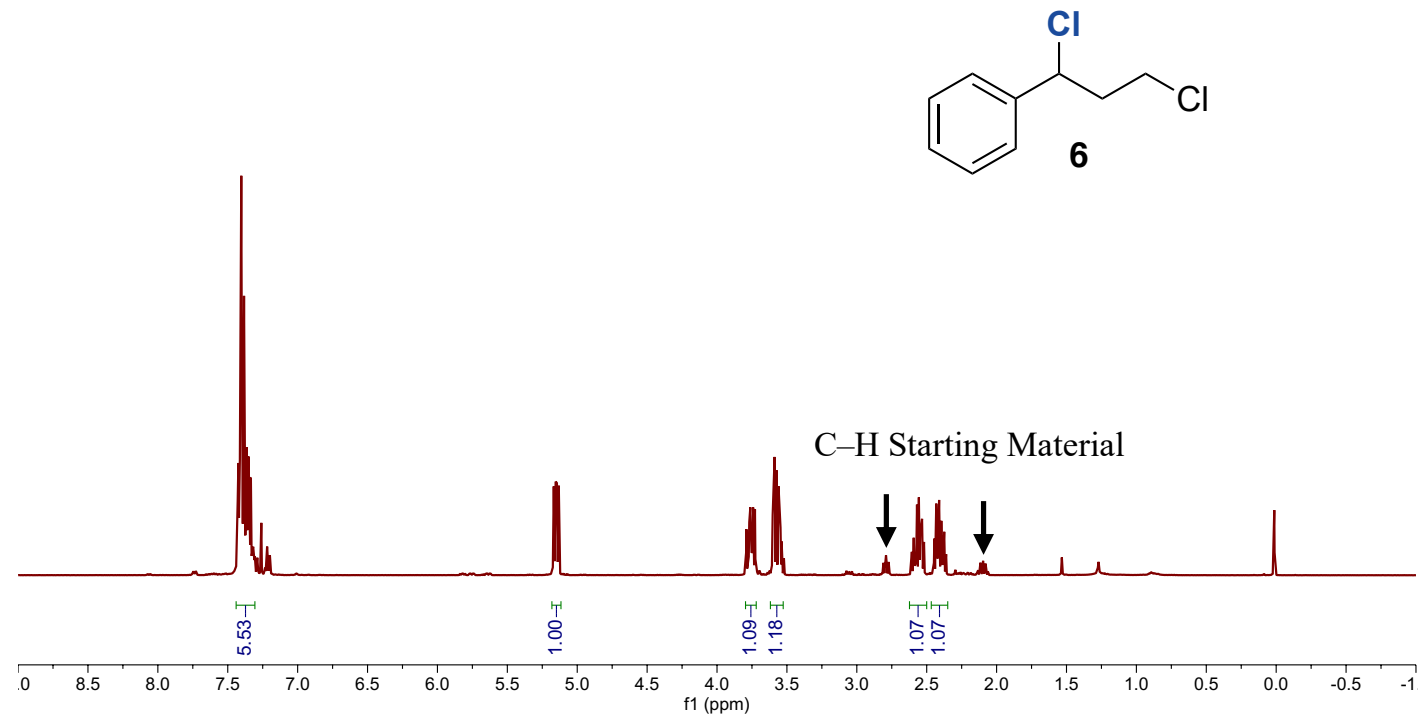

${ }^{13}$ C NMR $\left(101 \mathrm{MHz}, \mathrm{CDCl}_{3}\right)$ of 6

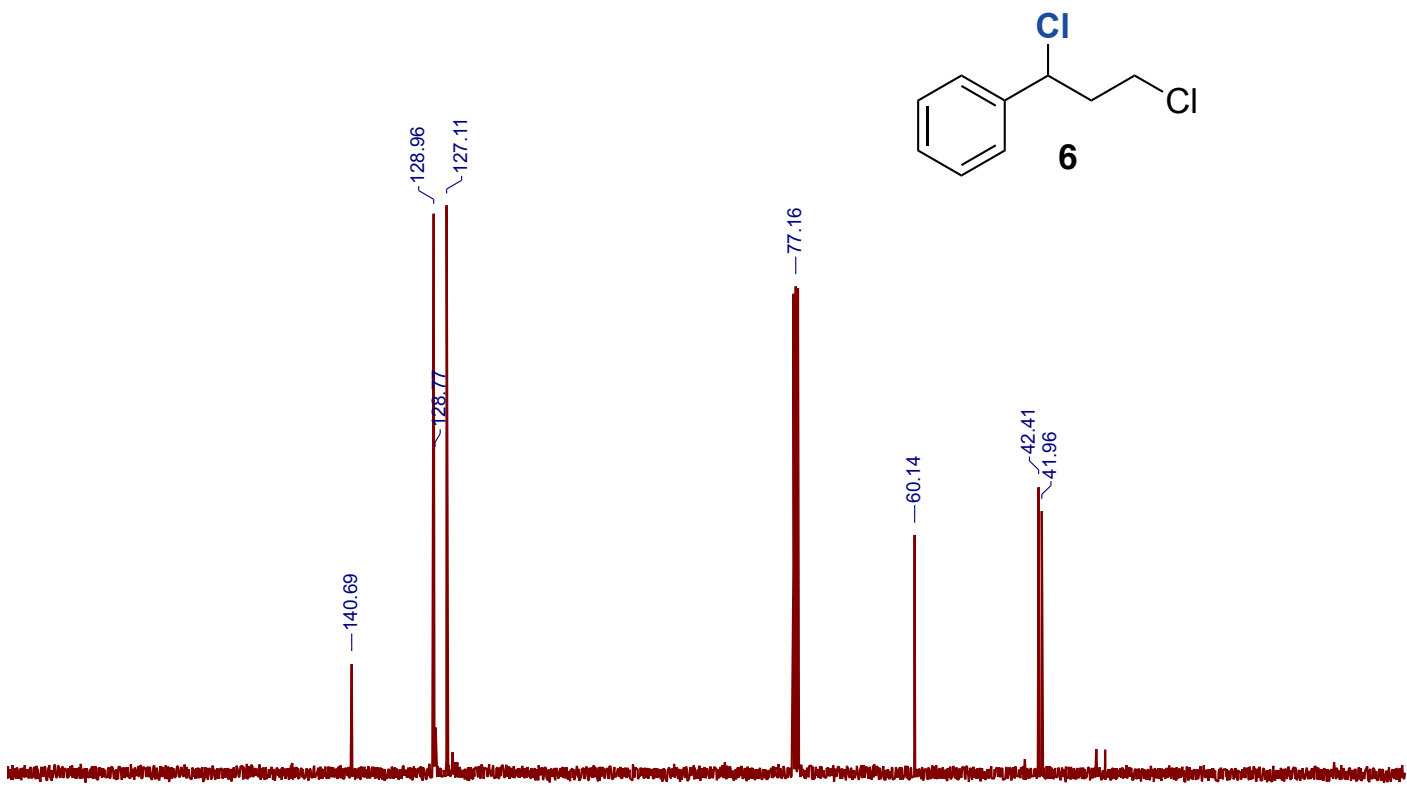

$\begin{array}{llll}180 & 170 & 160 & 150\end{array}$

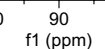


${ }^{1} \mathbf{H}$ NMR $\left(500 \mathrm{MHz}, \mathrm{CDCl}_{3}\right)$ of 7
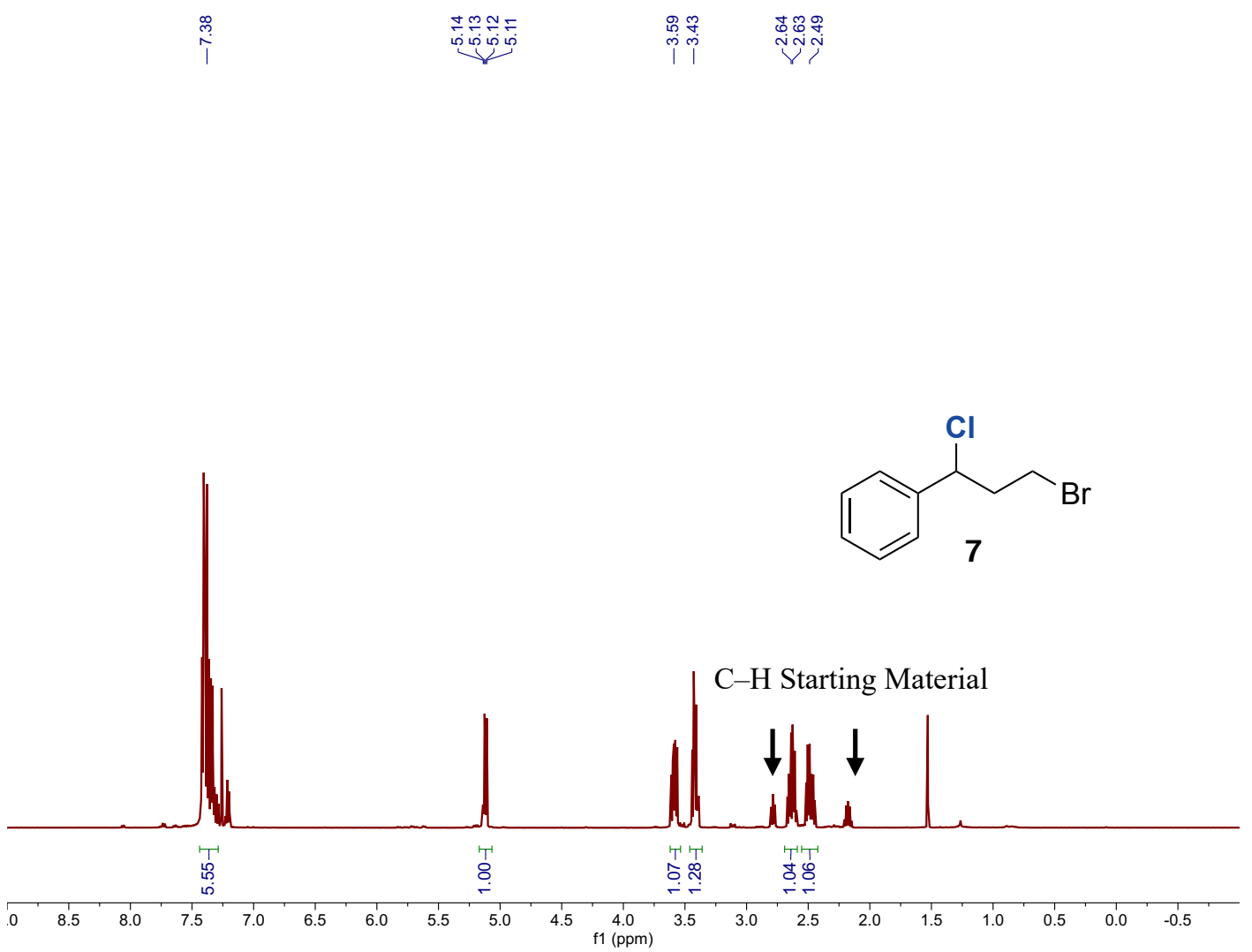

${ }^{13} \mathrm{C}$ NMR $\left(126 \mathrm{MHz}, \mathrm{CDCl}_{3}\right)$ of 7

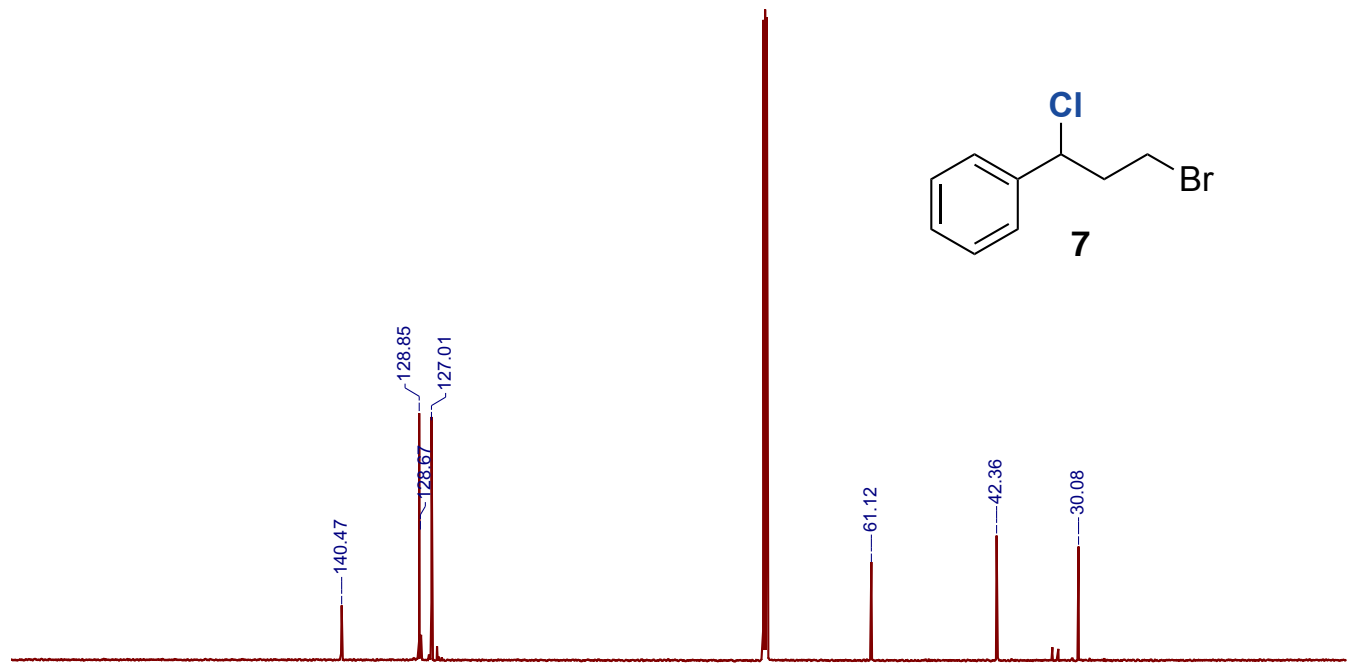

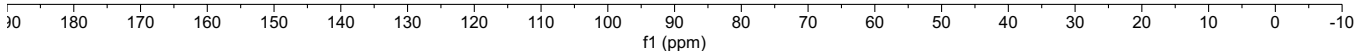


${ }^{1} \mathbf{H}$ NMR $\left(400 \mathrm{MHz}, \mathrm{CDCl}_{3}\right)$ of $\mathbf{8}$

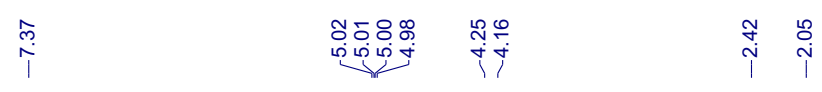

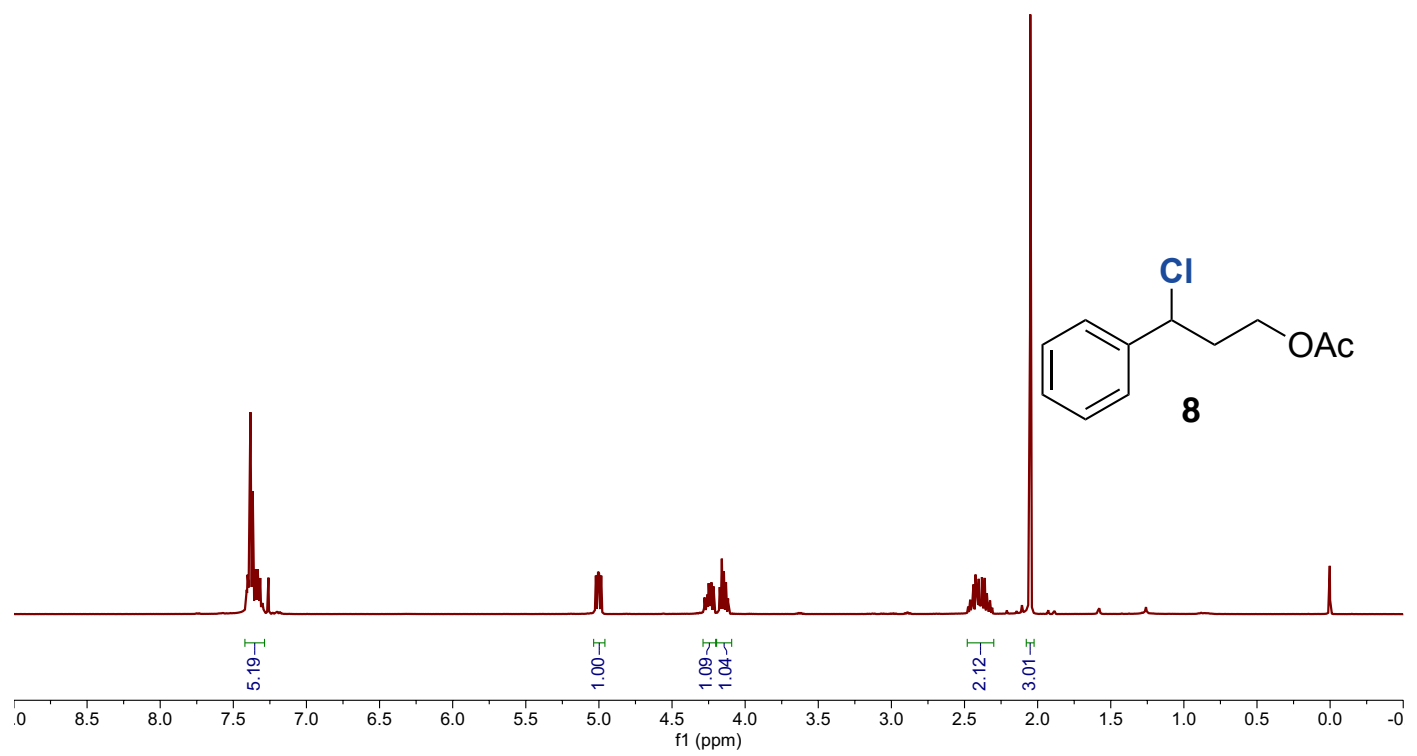

${ }^{13} \mathbf{C}$ NMR $\left(101 \mathrm{MHz}, \mathrm{CDCl}_{3}\right)$ of 8<smiles>CC(=O)OCCC(Cl)c1ccccc1</smiles>

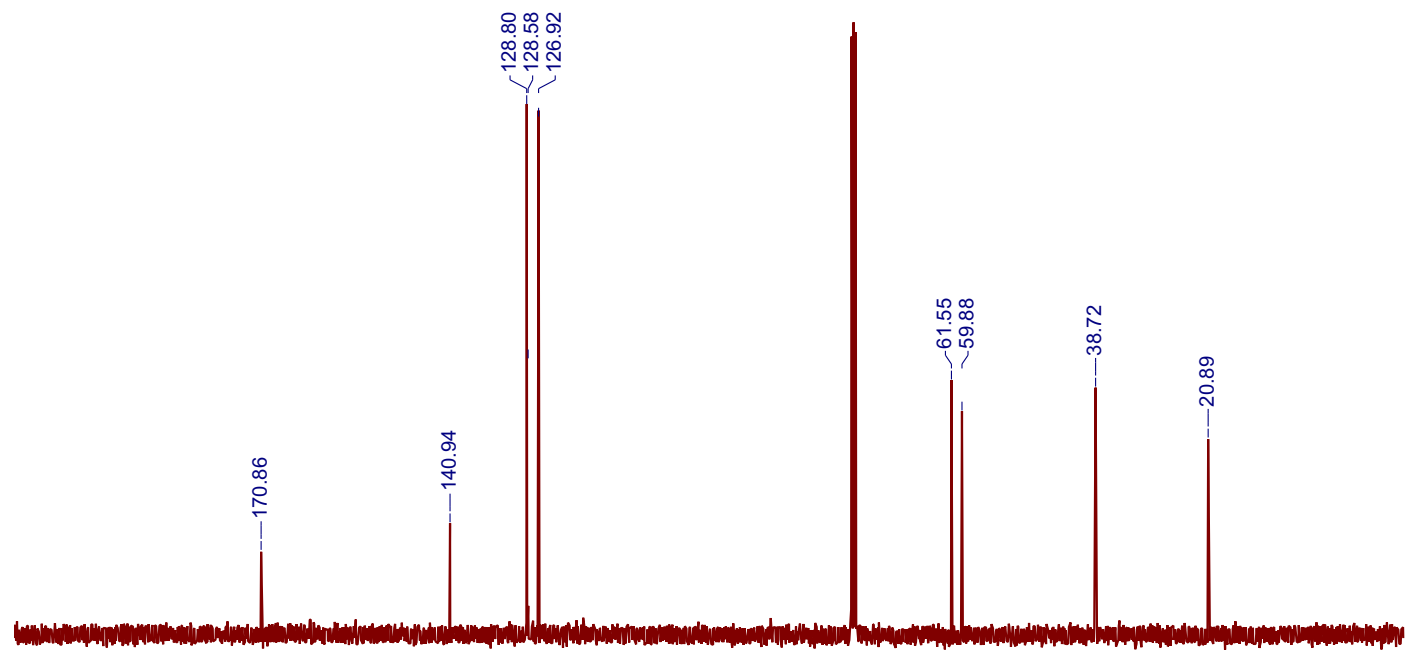

\begin{tabular}{lllllllllllllllllllllll}
\hline 10 & 200 & 190 & 180 & 170 & 160 & 150 & 140 & 130 & 120 & 110 & $\begin{array}{c}1 \\
100\end{array}$ & 90 & 80 & 70 & 60 & 50 & 40 & 30 & 20 & 10 & 0 & -1
\end{tabular} 
${ }^{1} \mathbf{H}$ NMR $\left(500 \mathrm{MHz}, \mathrm{CDCl}_{3}\right)$ of 9

$$
\text { 离只导 }
$$

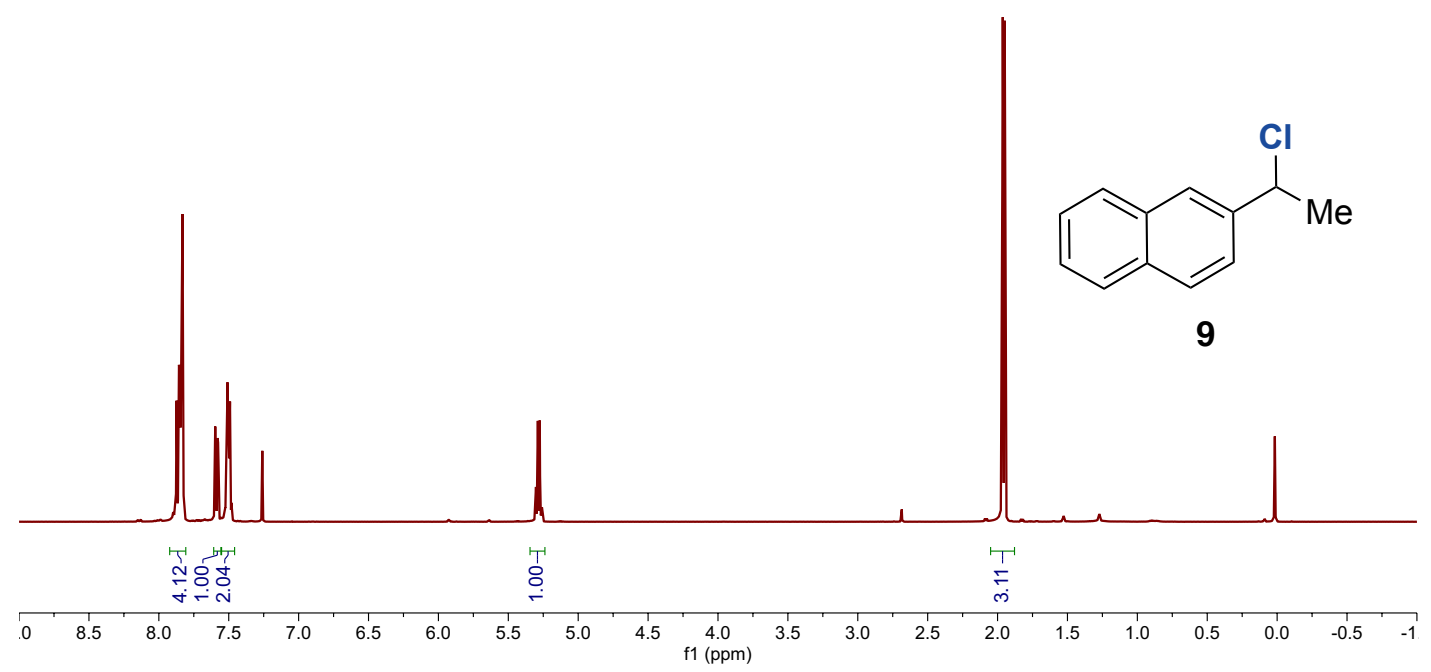

${ }^{13} \mathbf{C}$ NMR $\left(126 \mathrm{MHz}, \mathrm{CDCl}_{3}\right)$ of 9

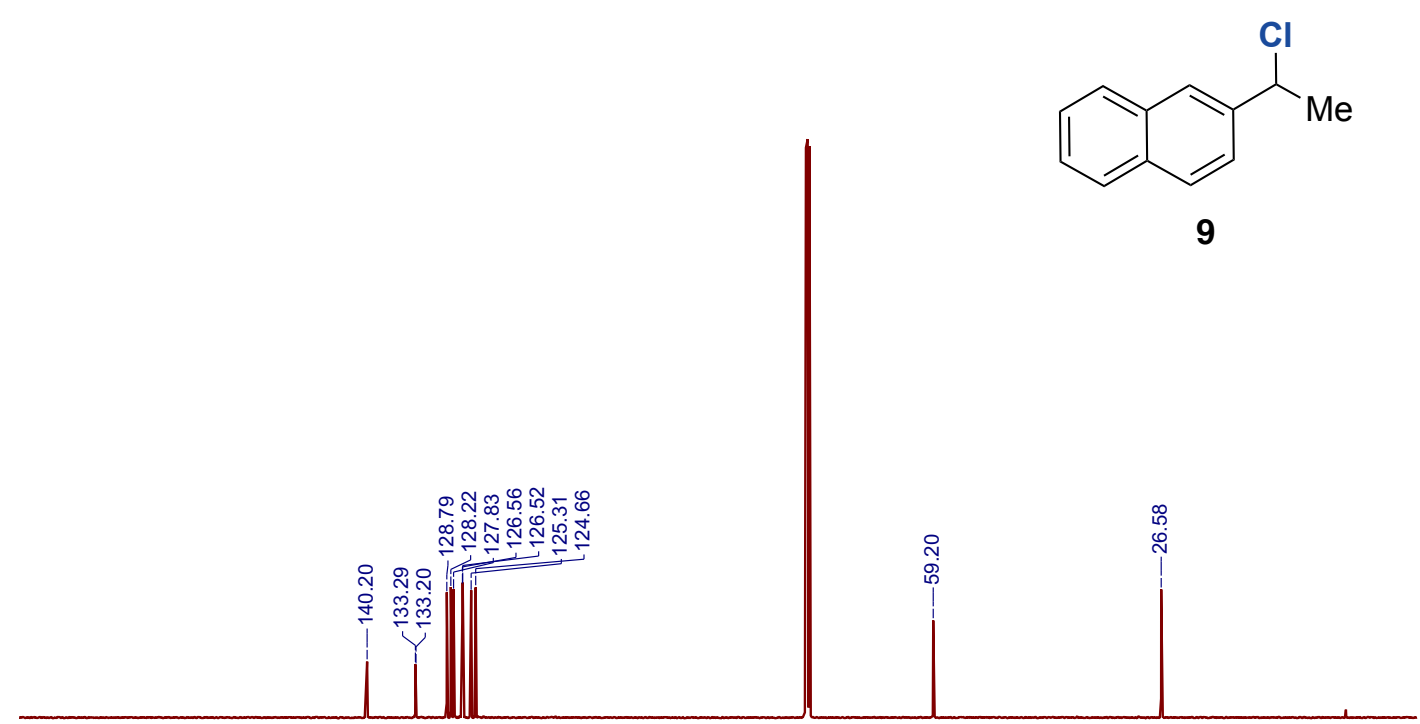

$\begin{array}{lllllllllllllllllllll}30 & 180 & 170 & 160 & 150 & 140 & 130 & 120 & 110 & 100 & \underset{f 1}{9(\mathrm{ppm})} & 80 & 70 & 60 & 50 & 40 & 30 & 20 & 10 & 0 & -1\end{array}$ 
${ }^{1} \mathbf{H}$ NMR $\left(500 \mathrm{MHz}, \mathrm{CDCl}_{3}\right)$ of $\mathbf{1 0}$

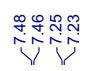

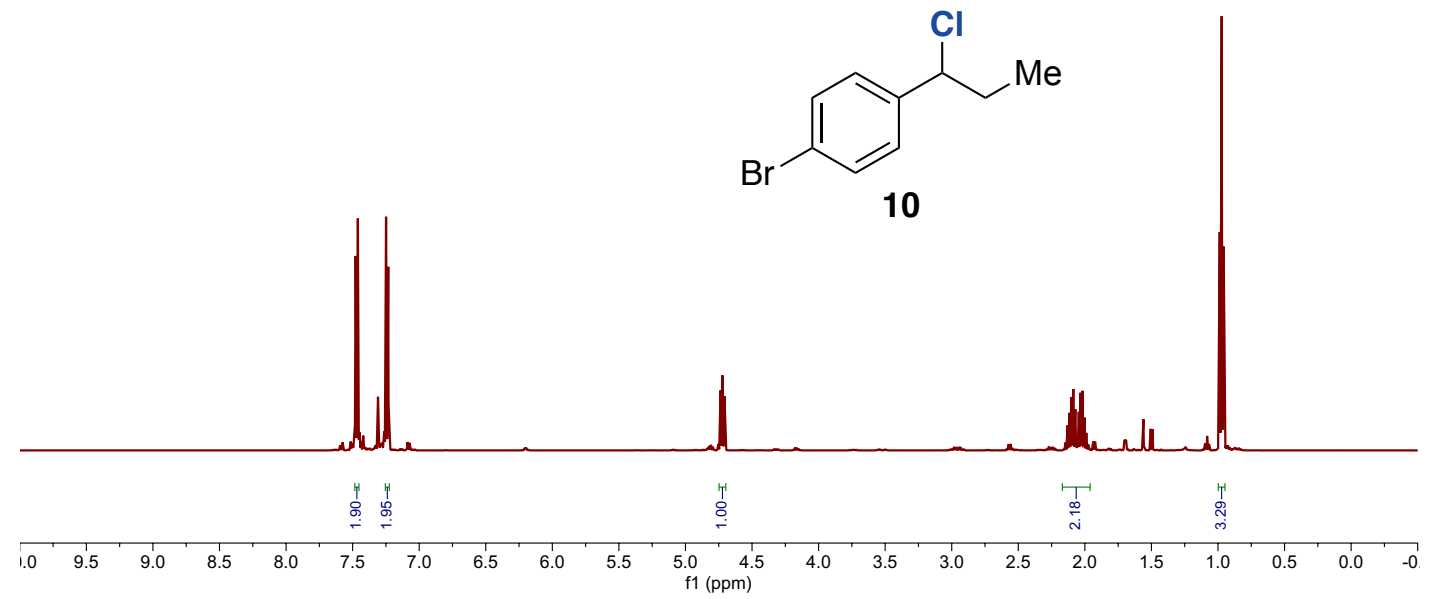

${ }^{13} \mathbf{C ~ N M R}\left(126 \mathrm{MHz}, \mathrm{CDCl}_{3}\right)$ of $\mathbf{1 0}$
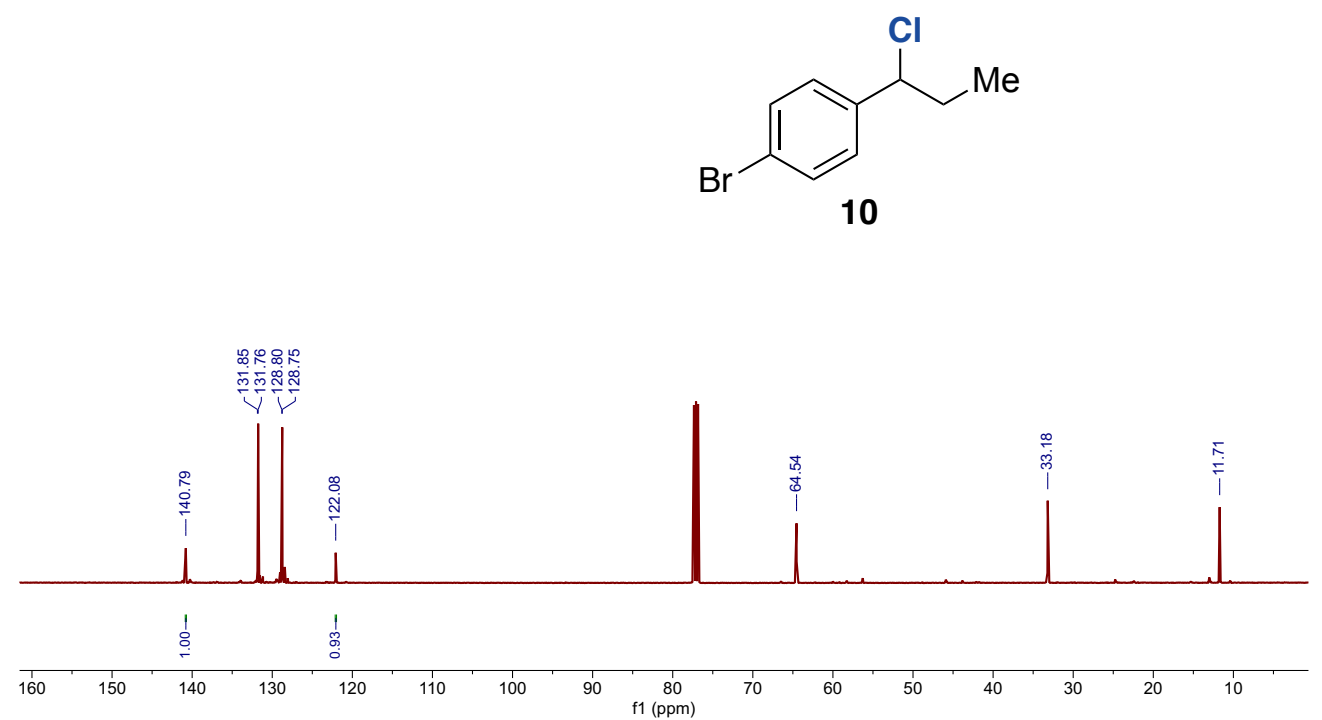
${ }^{1} \mathbf{H}$ NMR $\left(400 \mathrm{MHz}, \mathrm{CDCl}_{3}\right)$ of $\mathbf{1 1}$
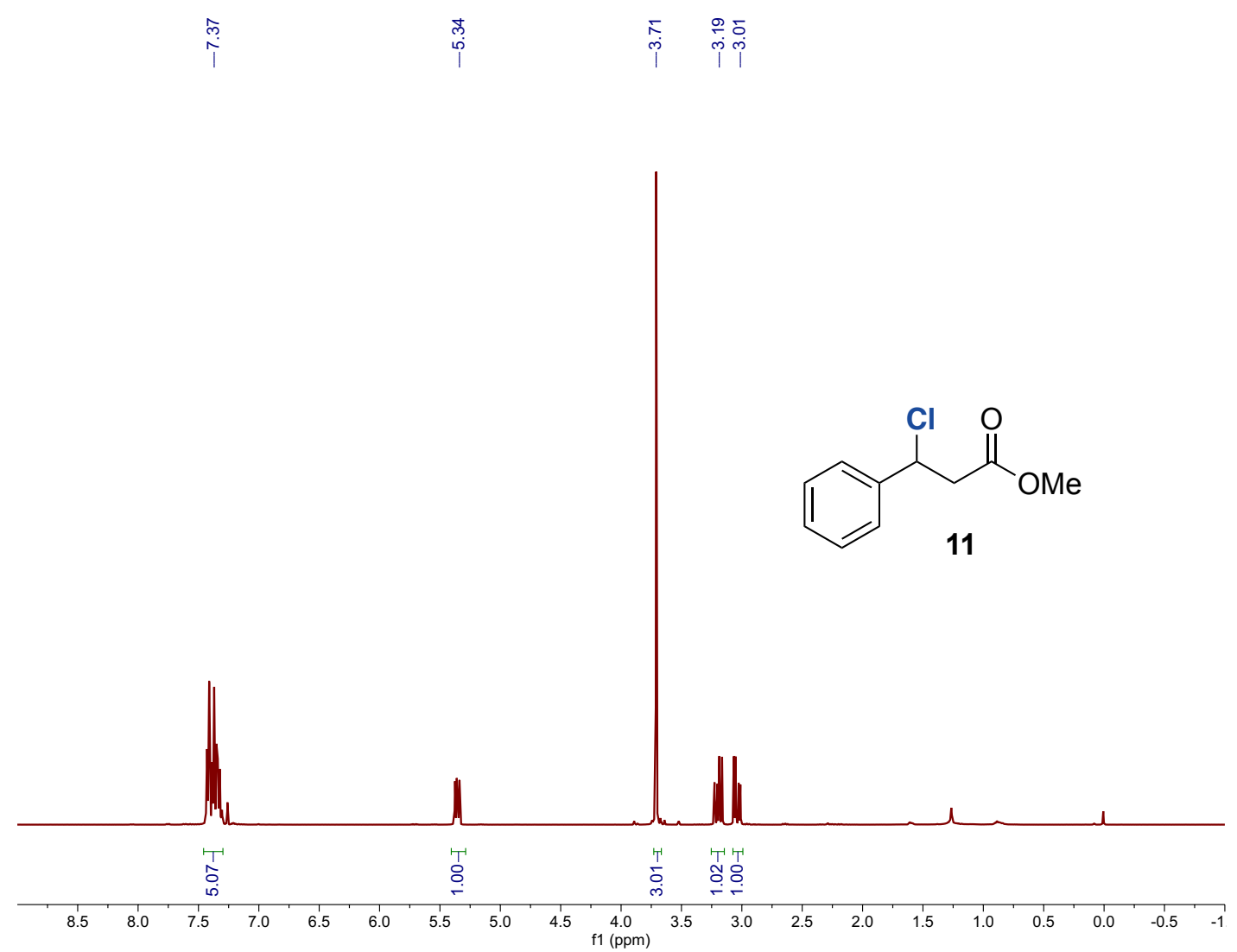

${ }^{13}$ C NMR $\left(101 \mathrm{MHz}, \mathrm{CDCl}_{3}\right)$ of $\mathbf{1 1}$

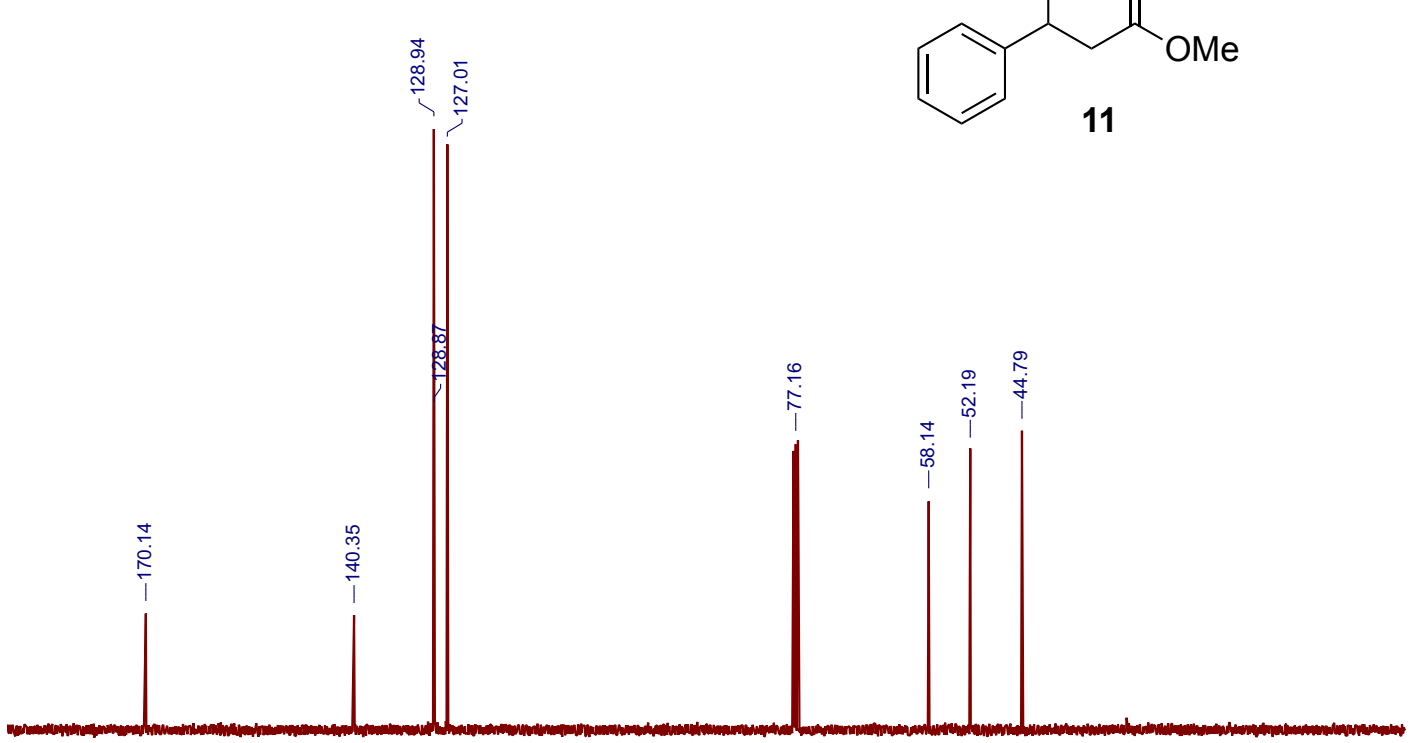

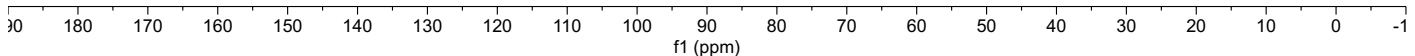


${ }^{\mathbf{1}} \mathbf{H}$ NMR $\left(400 \mathrm{MHz}, \mathrm{CDCl}_{3}\right)$ of $\mathbf{1 2}$
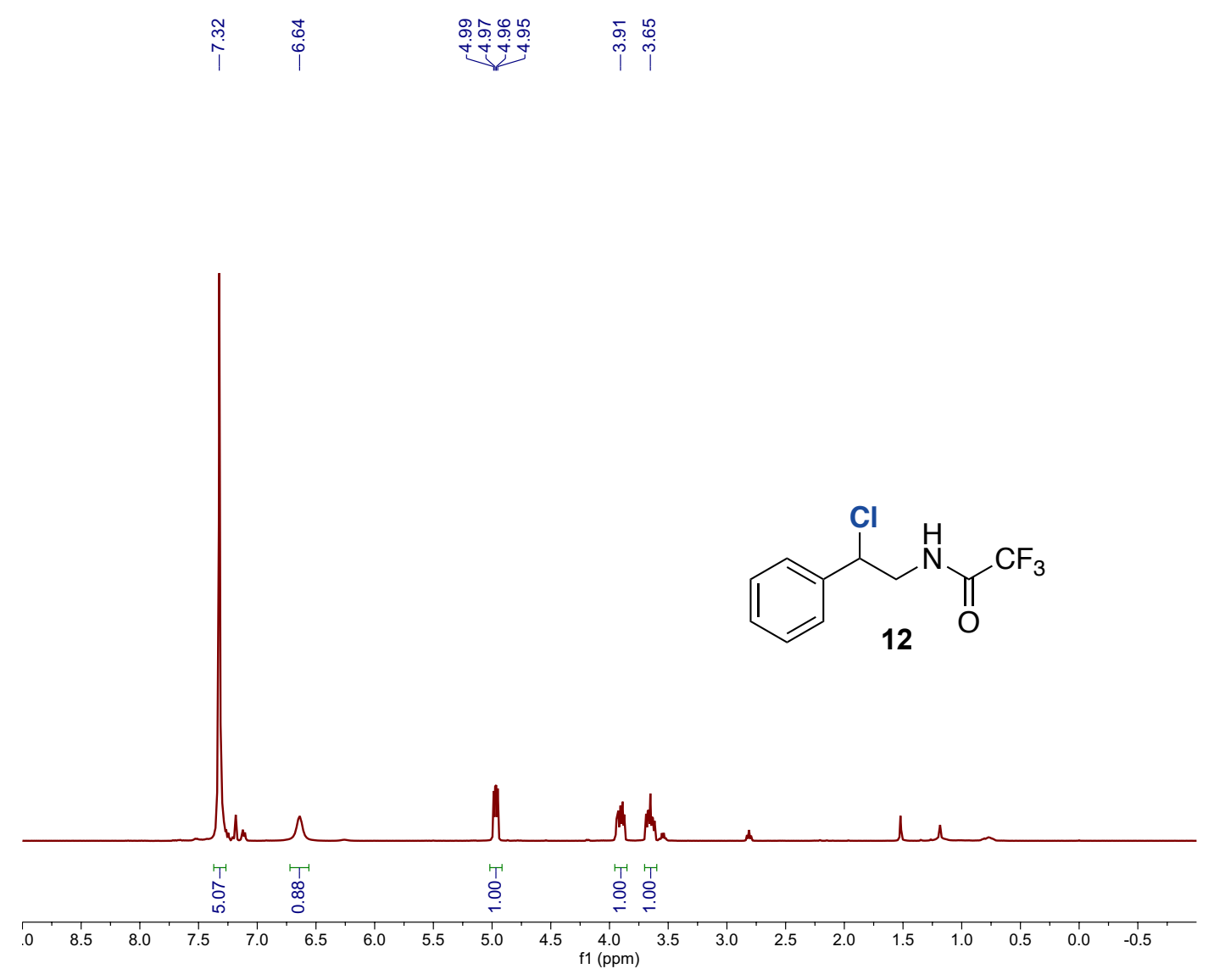

${ }^{13}$ C NMR $\left(101 \mathrm{MHz}, \mathrm{CDCl}_{3}\right)$ of $\mathbf{1 2}$

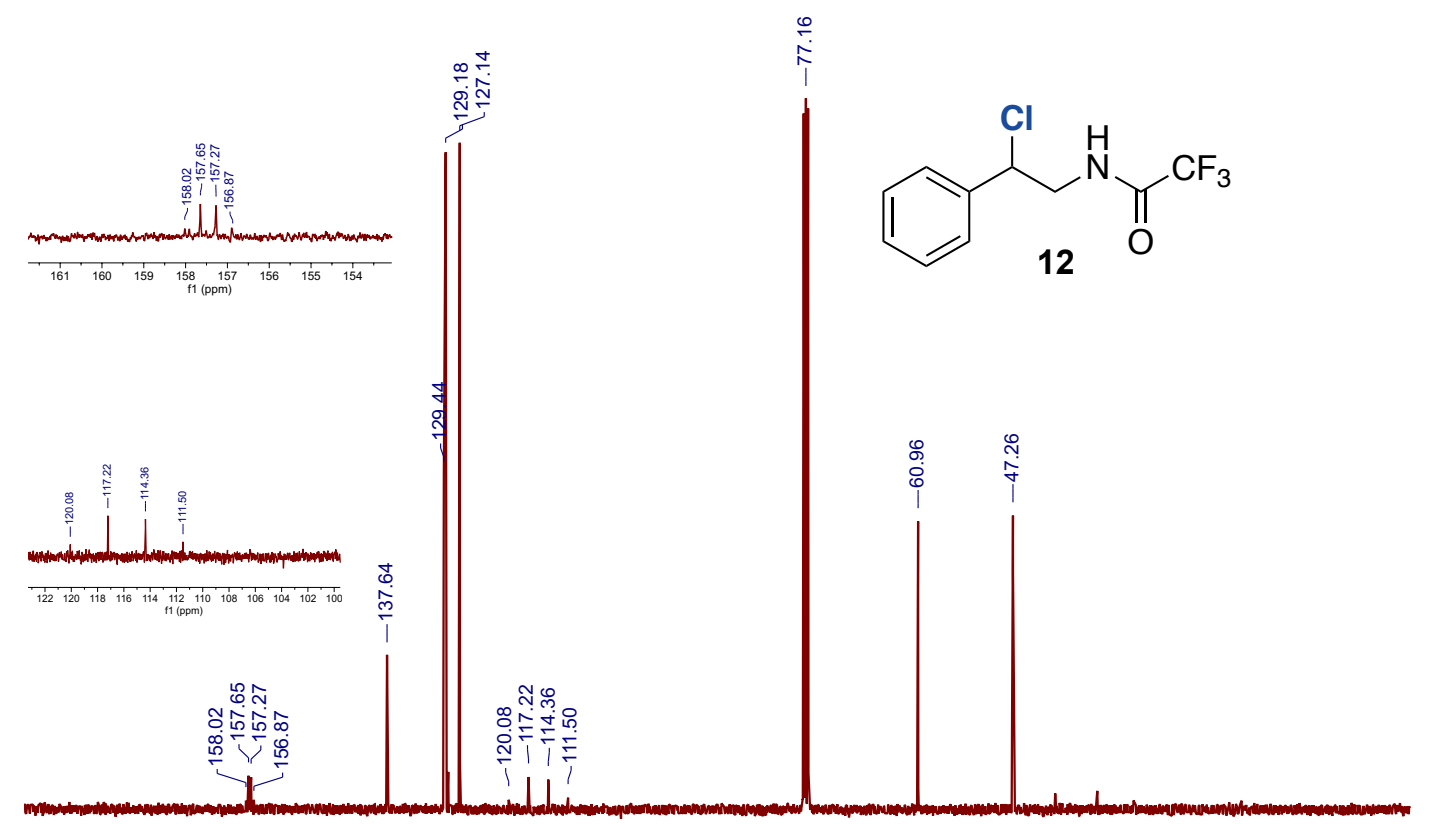

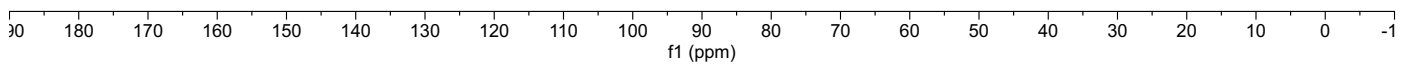


${ }^{19}$ F NMR (377 MHz, Chloroform- $d$ ) of 12

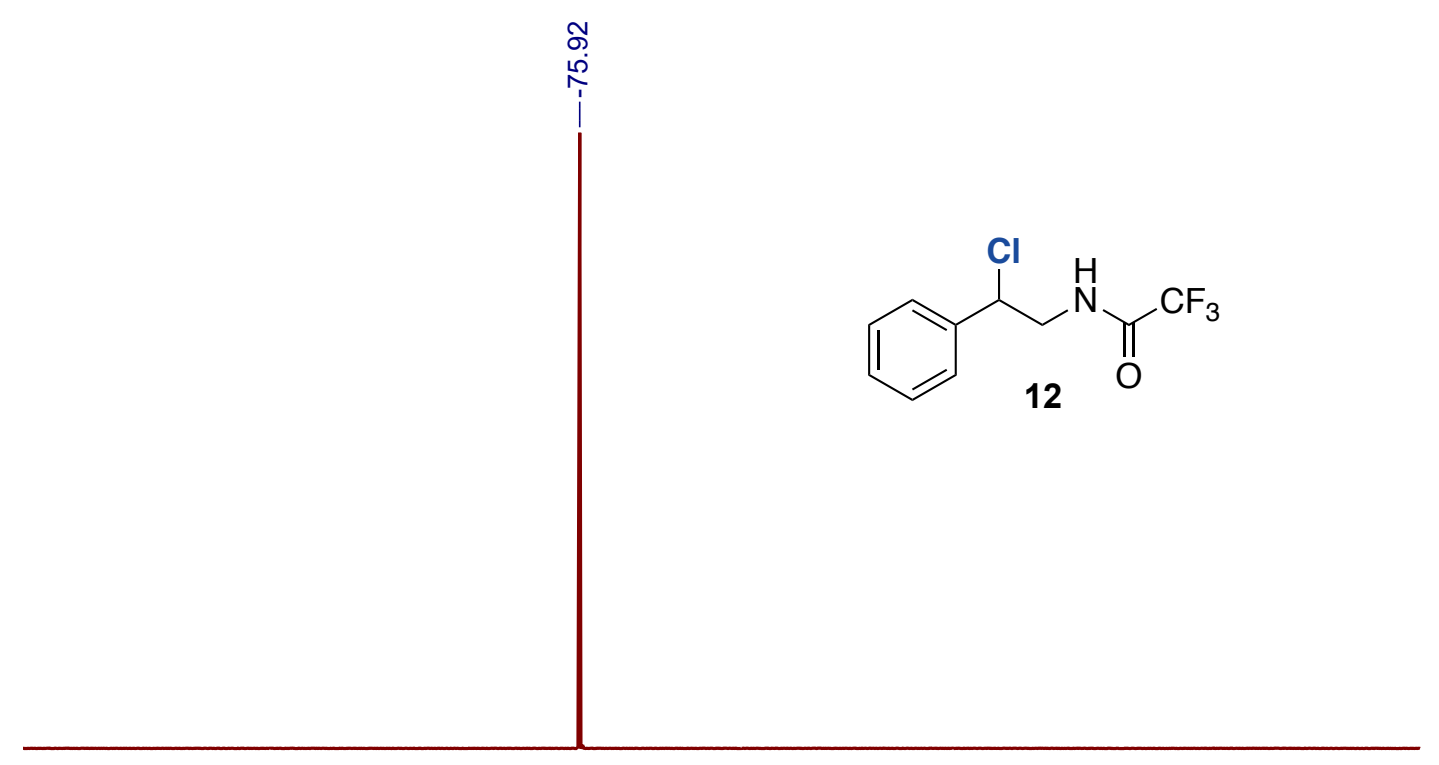

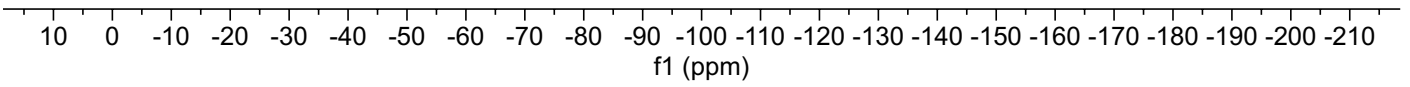


${ }^{1} \mathbf{H}$ NMR $\left(500 \mathrm{MHz}, \mathrm{CDCl}_{3}\right)$ of $\mathbf{1 3}$

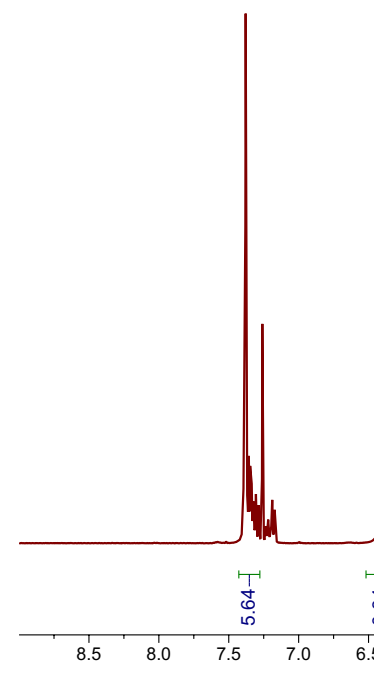<smiles>O=C(NCCC(Cl)c1ccccc1)C(F)(F)F</smiles>

13

${ }^{13} \mathbf{C ~ N M R ~}\left(126 \mathrm{MHz}, \mathrm{CDCl}_{3}\right)$ of $\mathbf{1 3}$
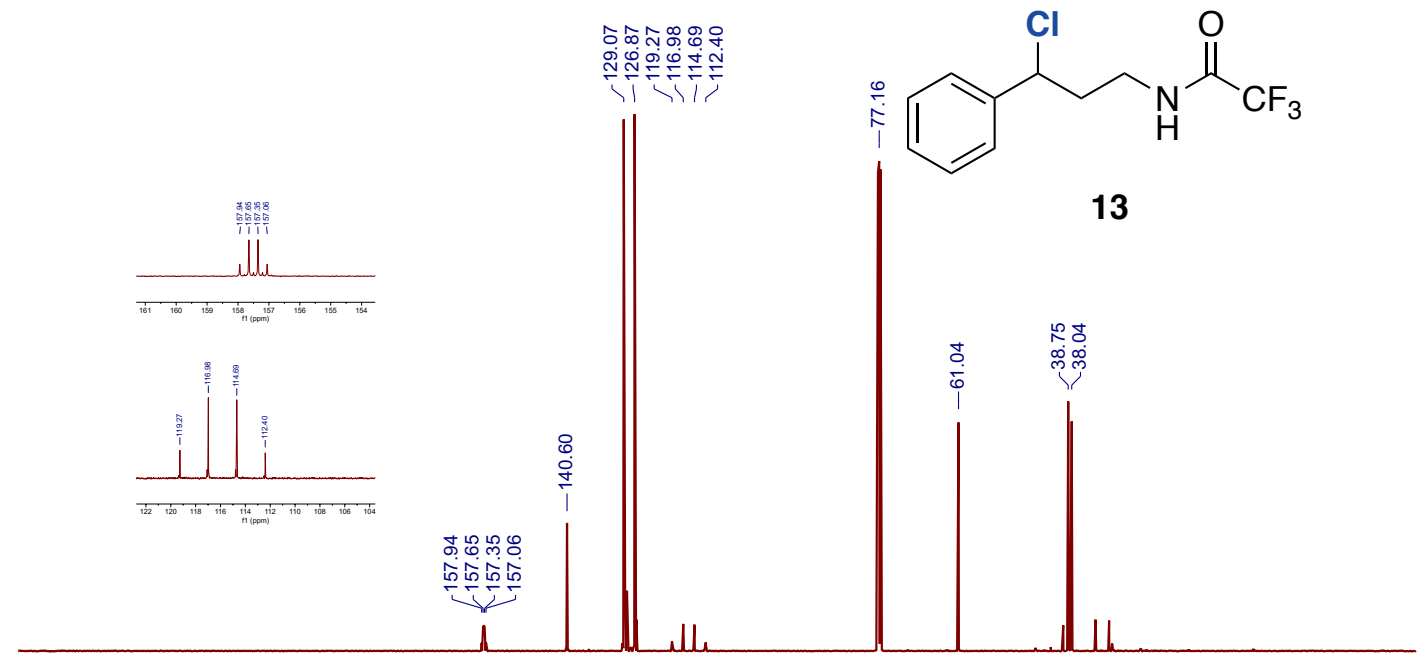

C-H Starting Material

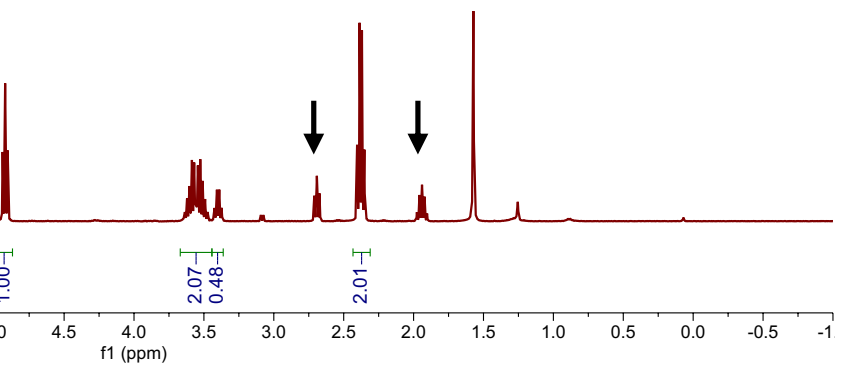

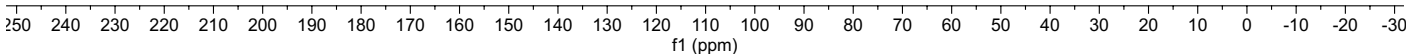


${ }^{19}$ F NMR (377 MHz, Chloroform-d) of 13
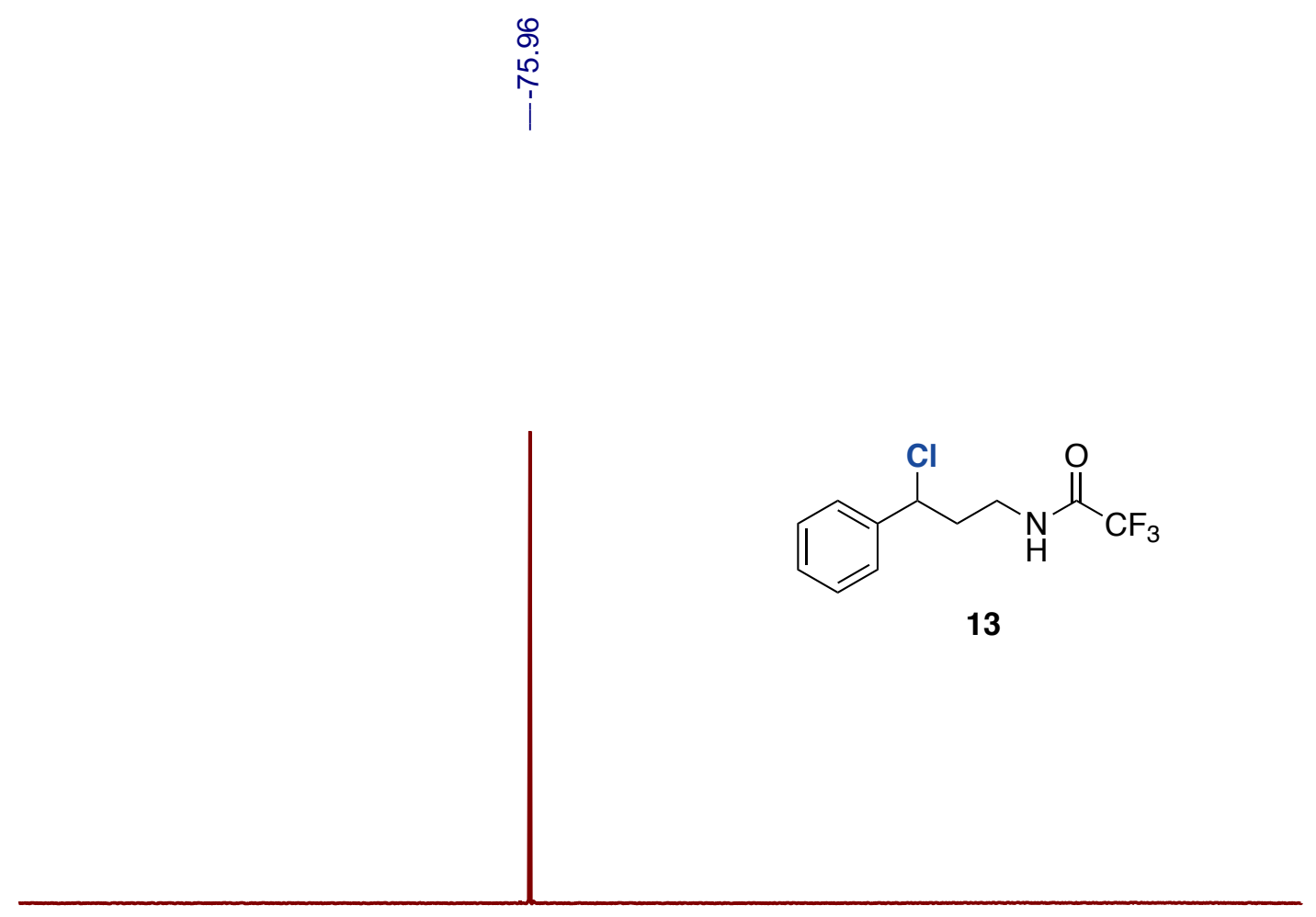

\begin{tabular}{lllllllllll}
\hline 0 & -20 & -40 & -60 & -80 & $\begin{array}{c}-100 \\
f 1(\mathrm{ppm})\end{array}$ & -120 & -140 & -160 & -180 & -200
\end{tabular}


${ }^{1} \mathbf{H}$ NMR $\left(500 \mathrm{MHz}, \mathrm{CDCl}_{3}\right)$ of $\mathbf{1 4}$
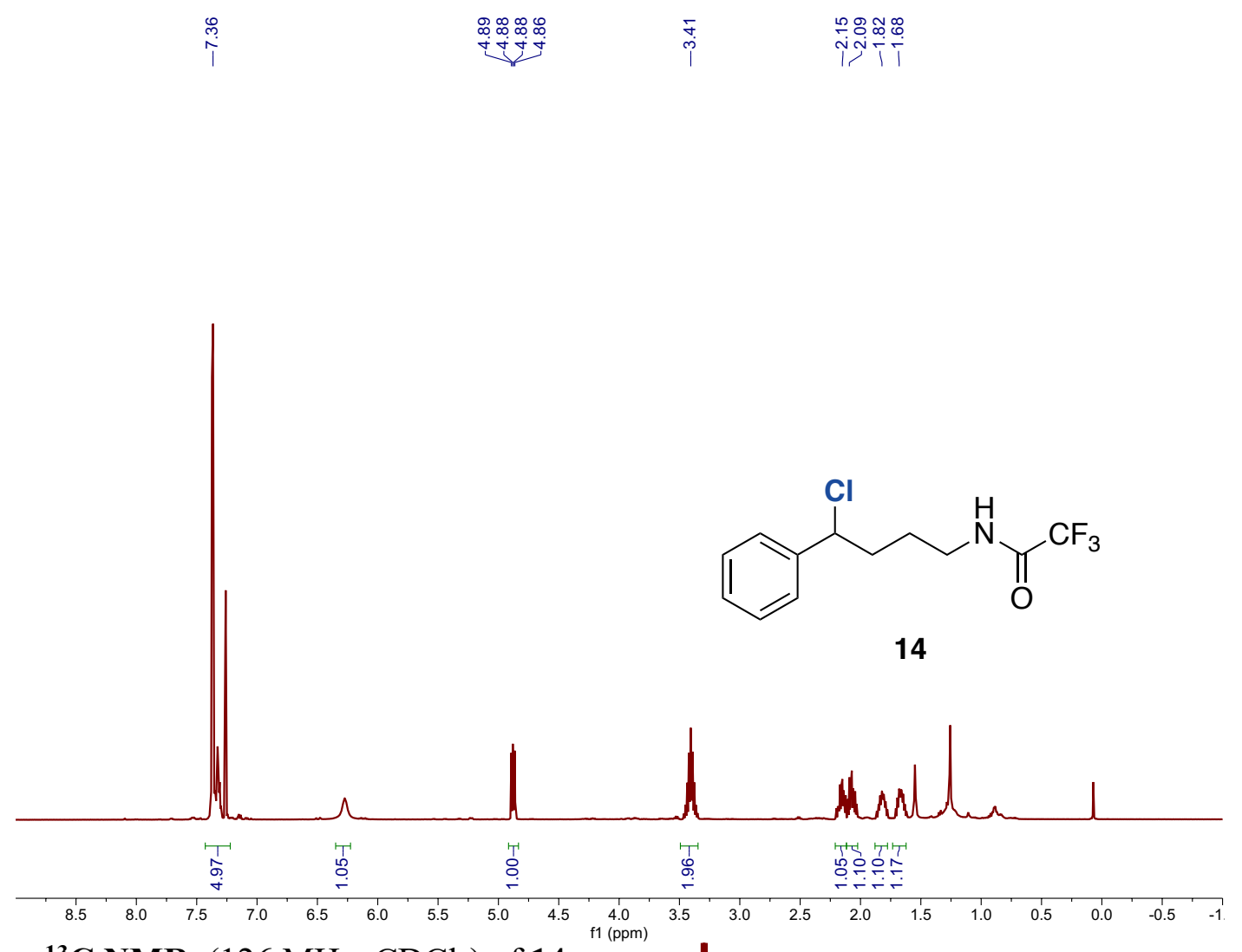

${ }^{13} \mathbf{C ~ N M R}\left(126 \mathrm{MHz}, \mathrm{CDCl}_{3}\right)$ of $\mathbf{1 4}$

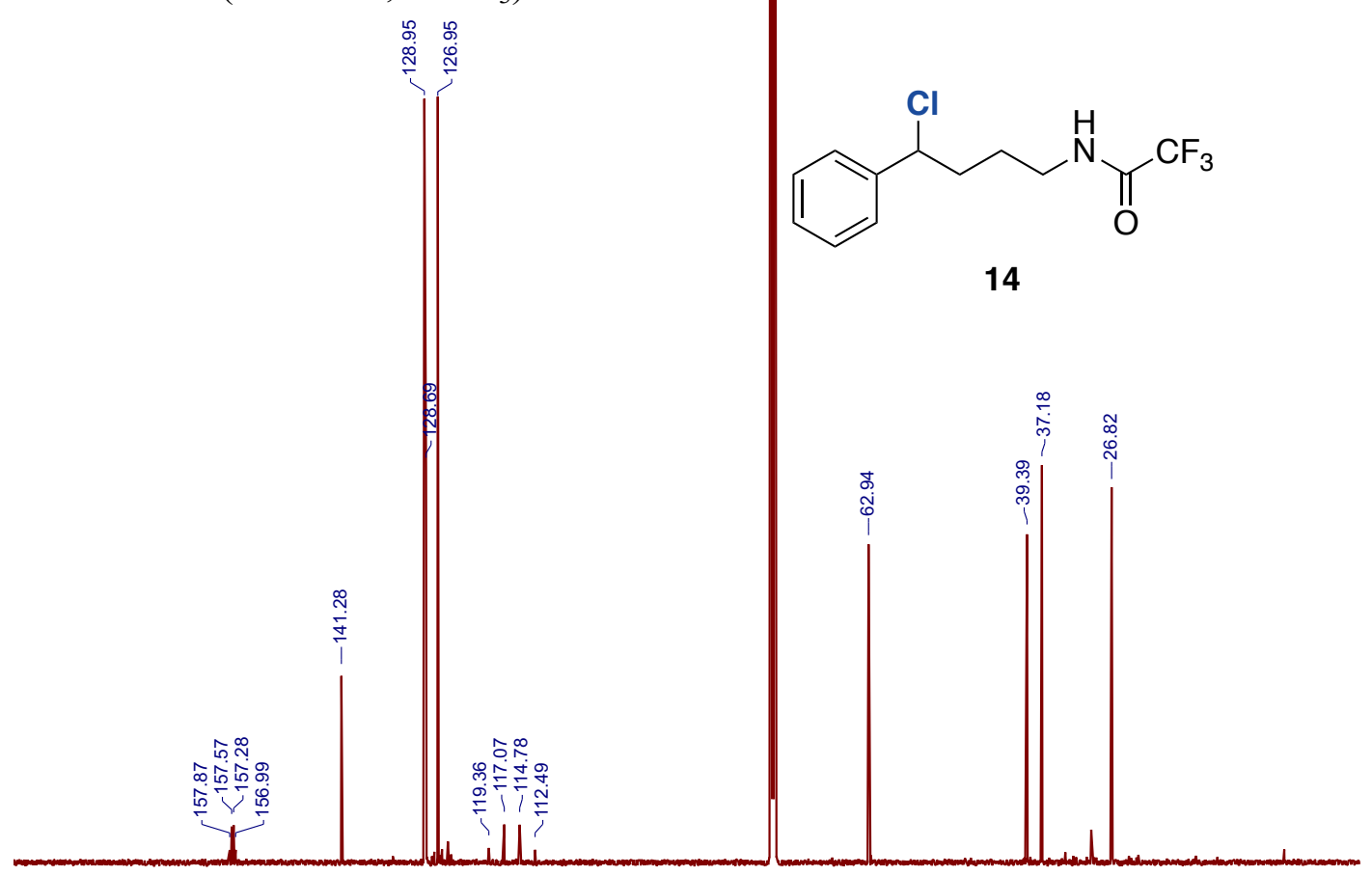

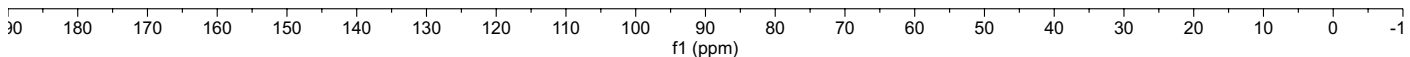


${ }^{19}$ F NMR (377 MHz, Chloroform- $d$ ) of 14

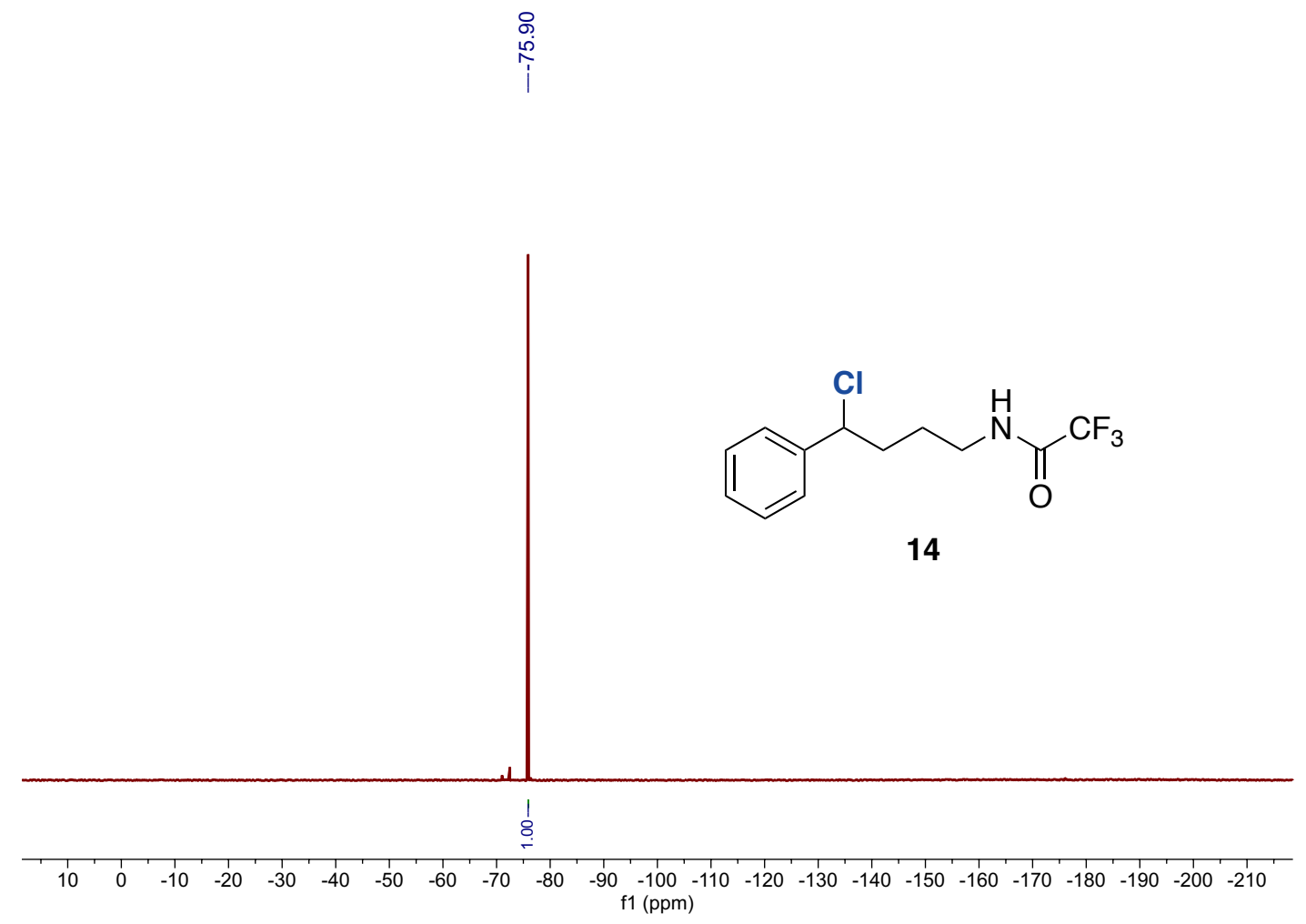


${ }^{1} \mathbf{H}$ NMR $\left(500 \mathrm{MHz}, \mathrm{CDCl}_{3}\right)$ of $\mathbf{1 5}$

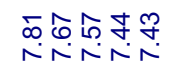

占行

i

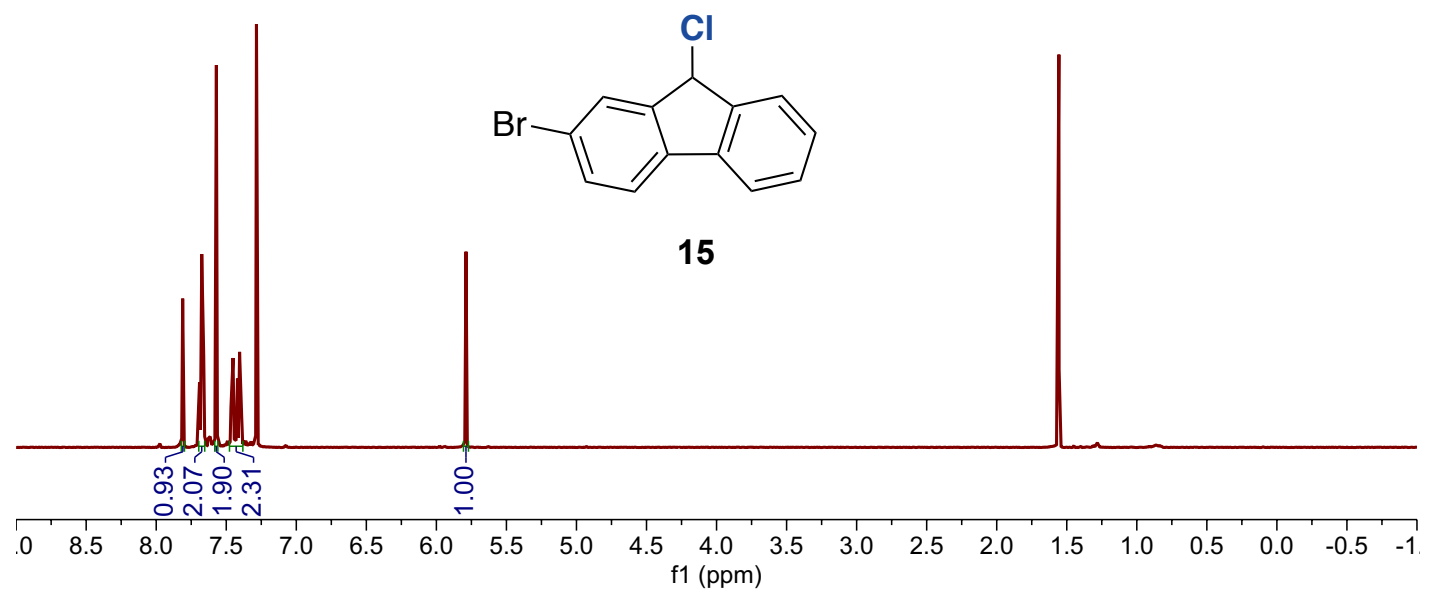

${ }^{13} \mathbf{C ~ N M R}\left(126 \mathrm{MHz}, \mathrm{CDCl}_{3}\right)$ of $\mathbf{1 5}$

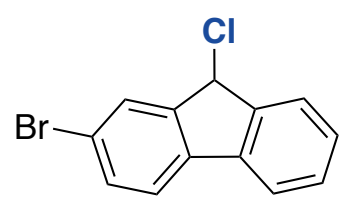

15

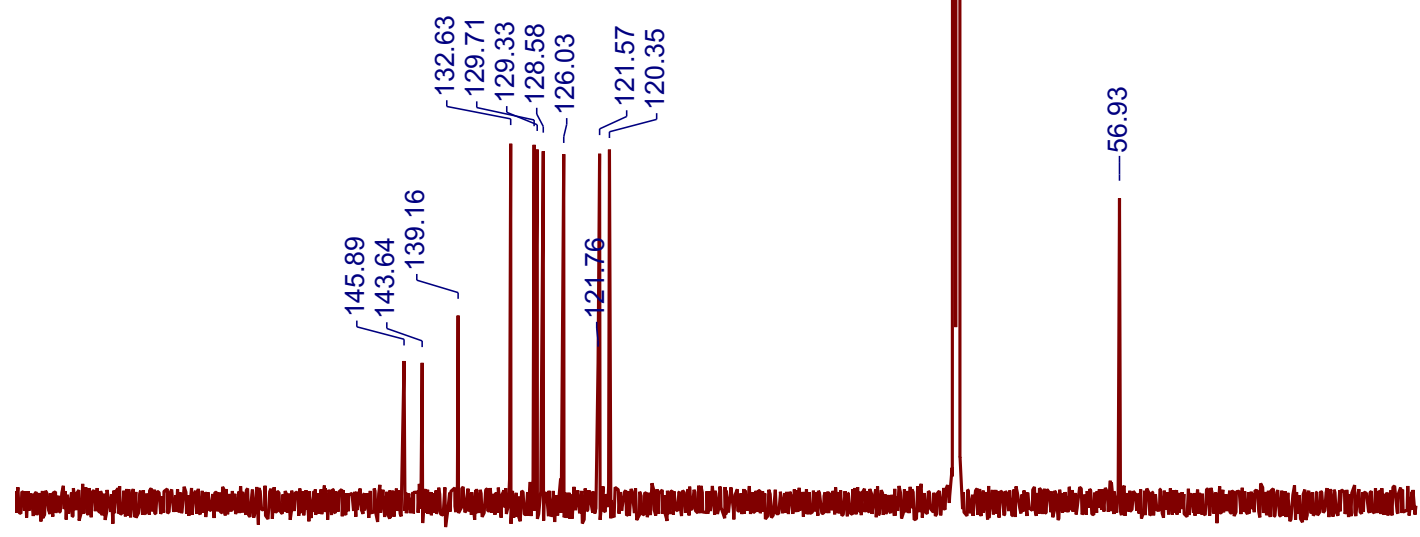

$\begin{array}{llllll}190 & 180 & 170 & 160 & 150 & 140\end{array}$

130120

110100 
${ }^{1} \mathbf{H}$ NMR (400 MHz, $\left.\mathrm{CDCl}_{3}\right)$ of 16
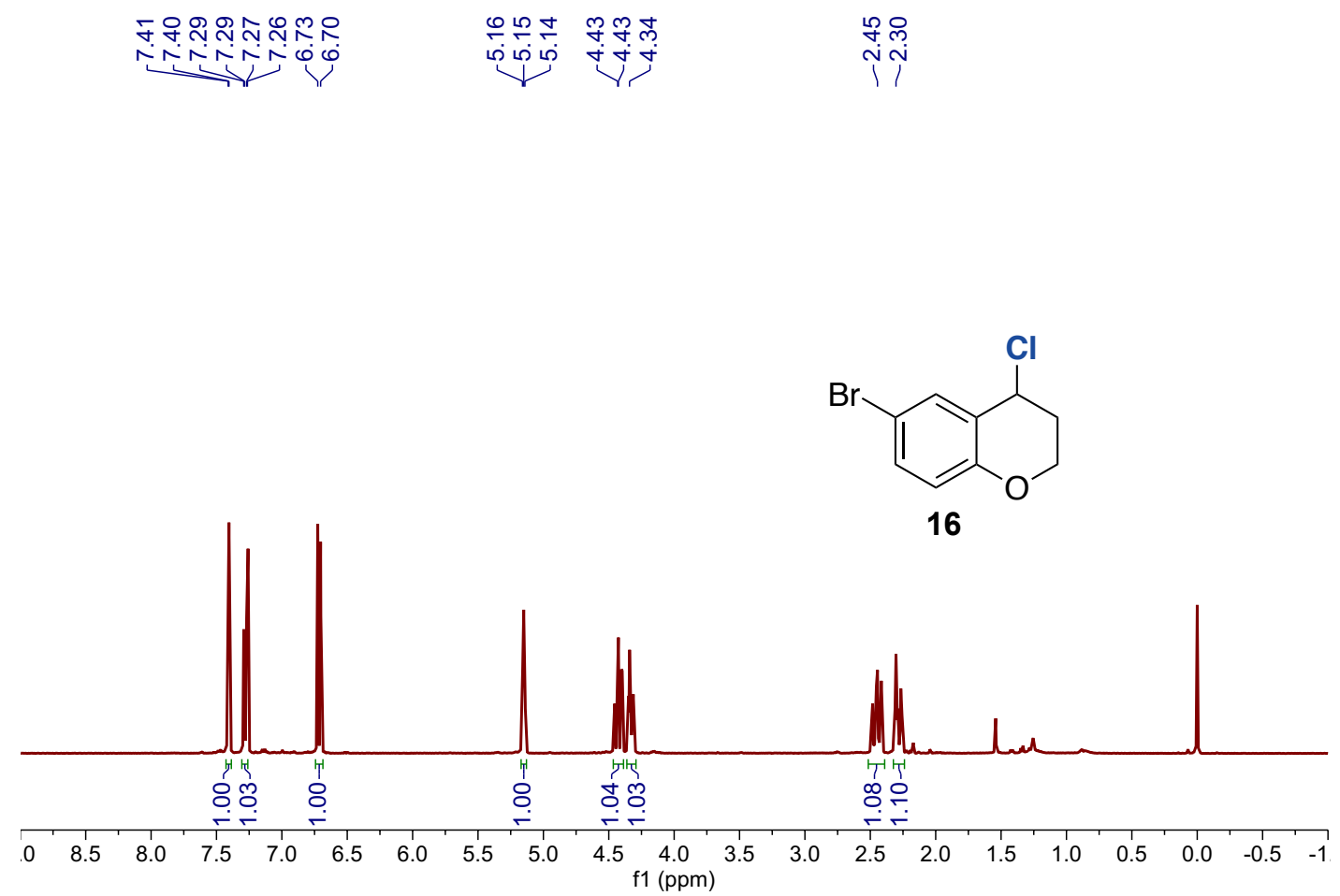

${ }^{13}$ C NMR (101 MHz, $\left.\mathrm{CDCl}_{3}\right)$ of 16

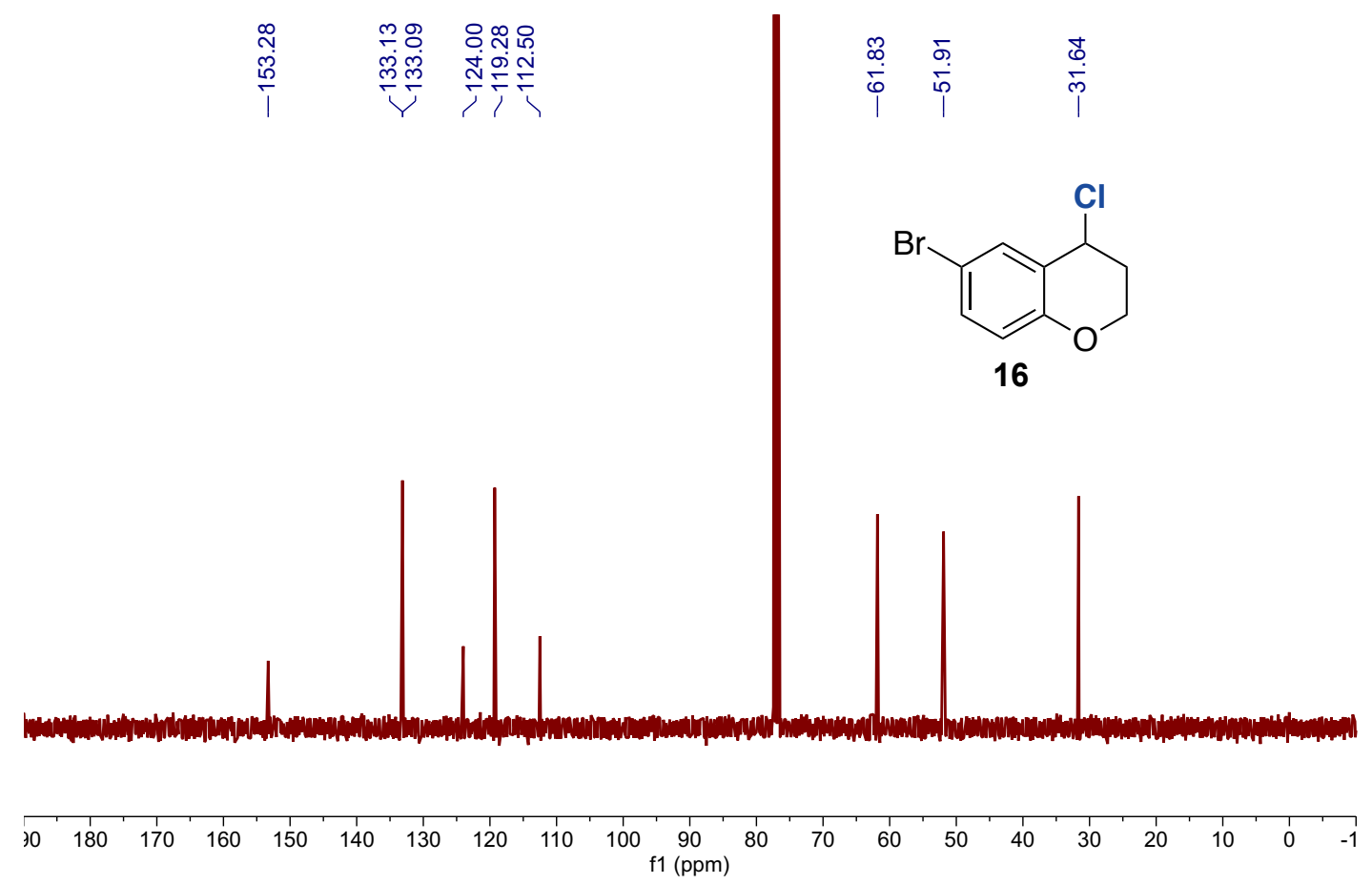


${ }^{1} \mathbf{H}$ NMR (400 MHz, $\left.\mathrm{CDCl}_{3}\right)$ of $\mathbf{1 7}$

\begin{tabular}{|c|c|c|}
\hline 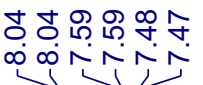 & 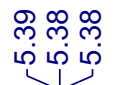 & 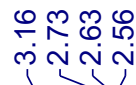 \\
\hline
\end{tabular}

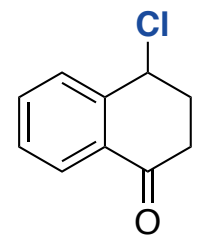

17

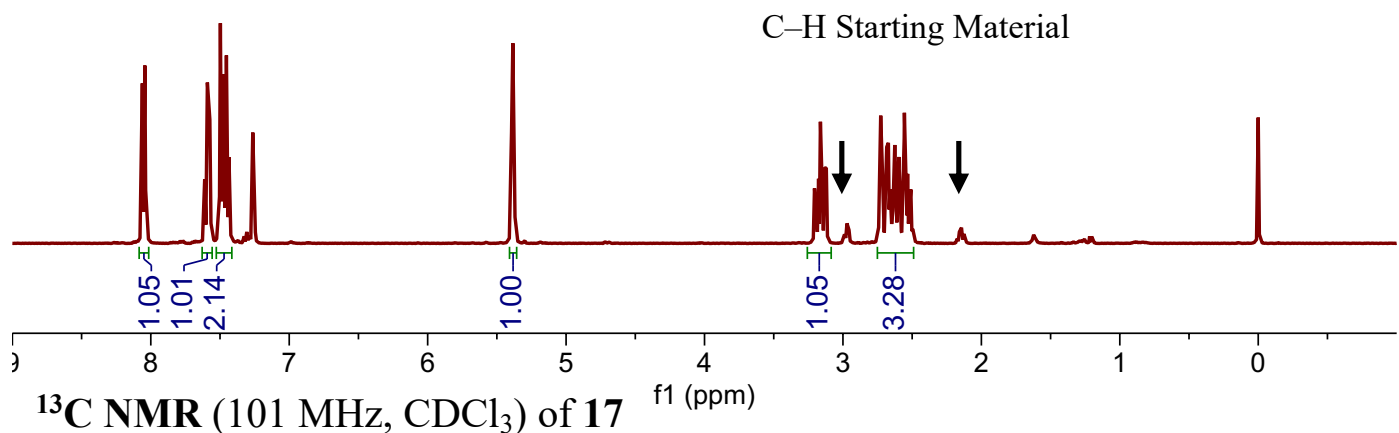

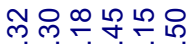

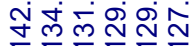

$\sqrt{-15}$

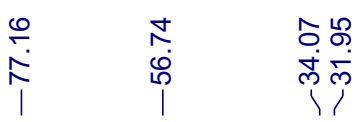

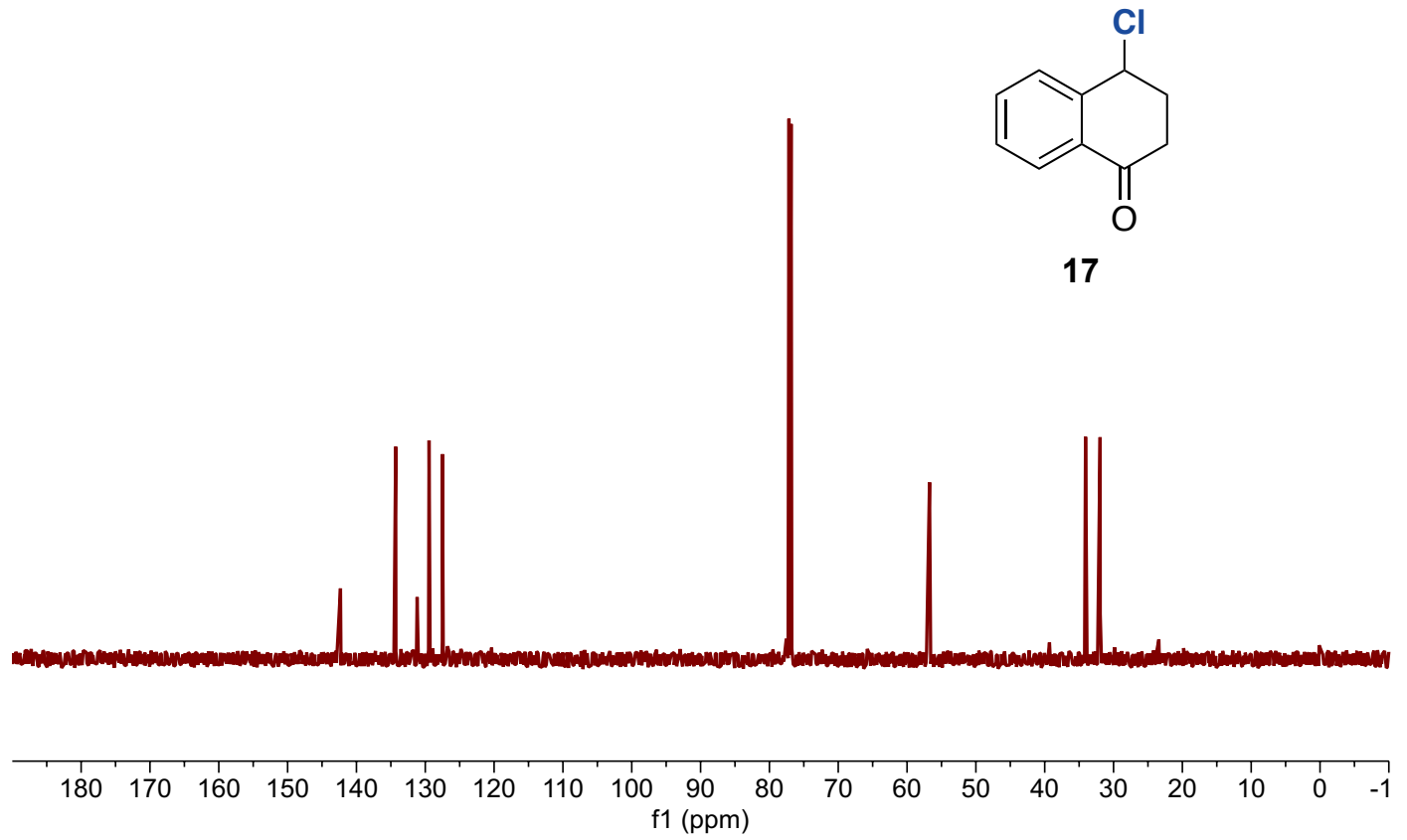


${ }^{1} \mathbf{H}$ NMR $\left(600 \mathrm{MHz}, \mathrm{CDCl}_{3}\right)$ of $\mathbf{1 8 a}$
$\stackrel{\substack{i \\ i}}{i}$

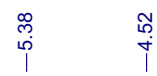

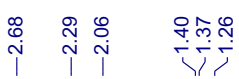<smiles>CC(=O)c1cc(C(C)(C)C)cc2c1C(O)CC2(C)C</smiles>

$18 a$

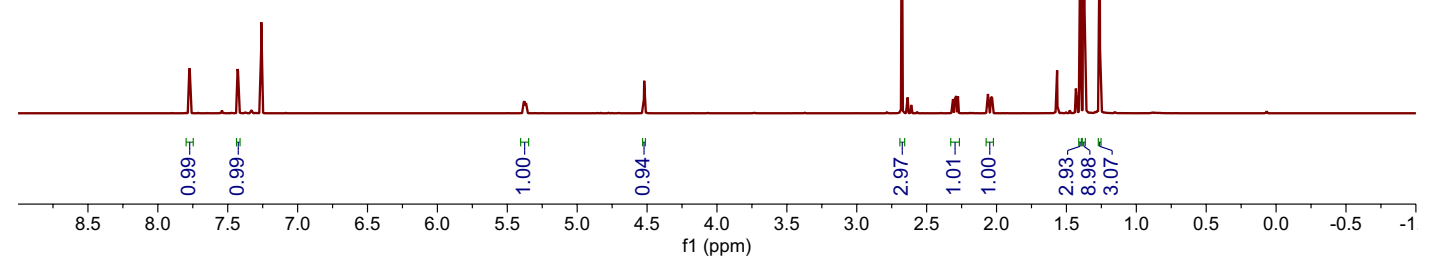

${ }^{13} \mathbf{C}$ NMR $\left(151 \mathrm{MHz}, \mathrm{CDCl}_{3}\right)$ of $\mathbf{1 8 a}$

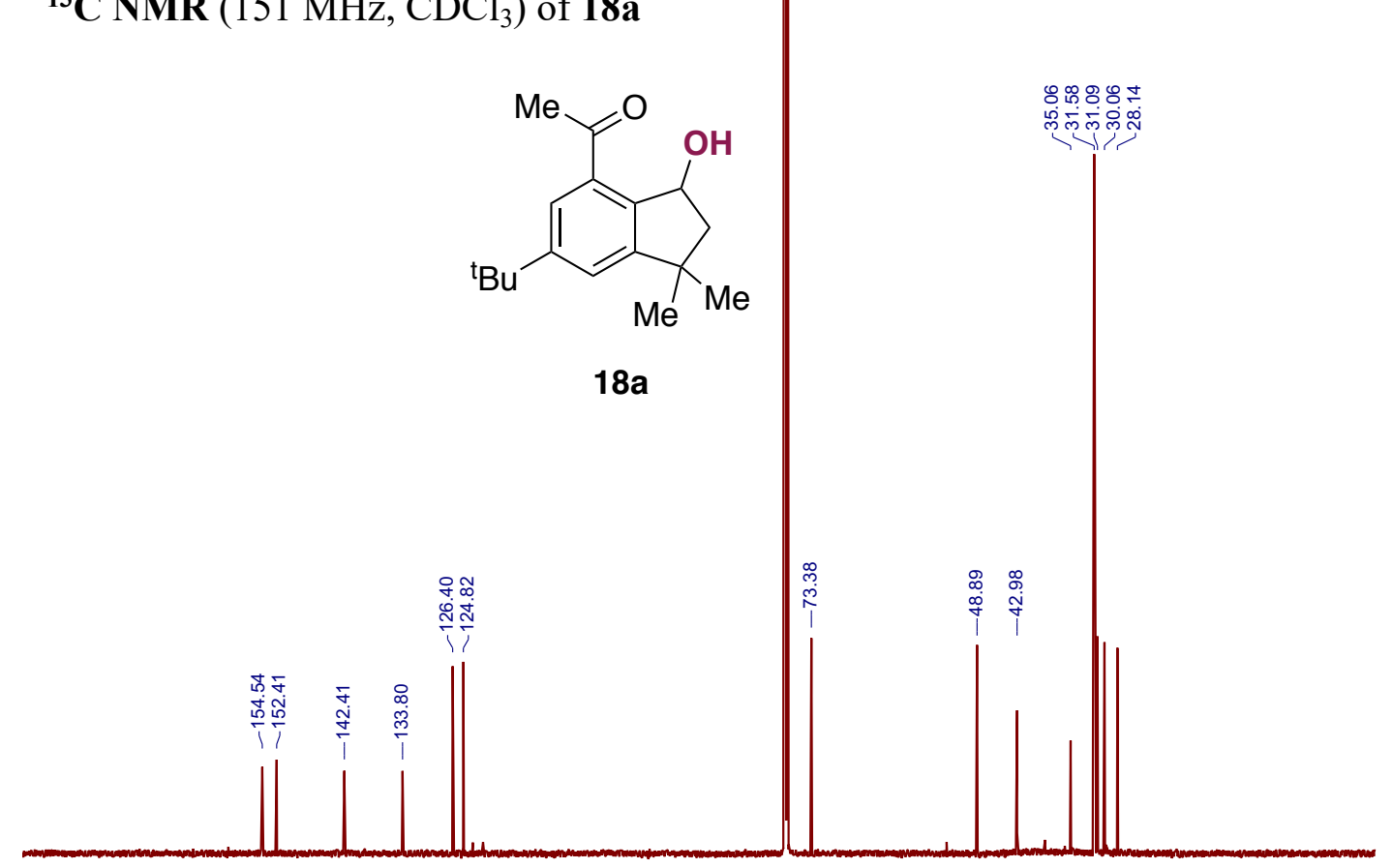

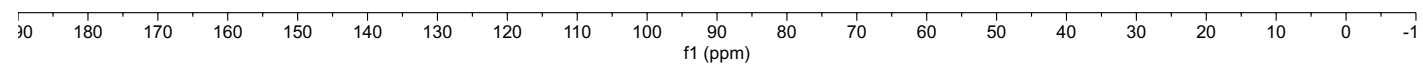


${ }^{1} \mathbf{H}$ NMR (400 MHz, $\left.\mathrm{CDCl}_{3}\right)$ of 19
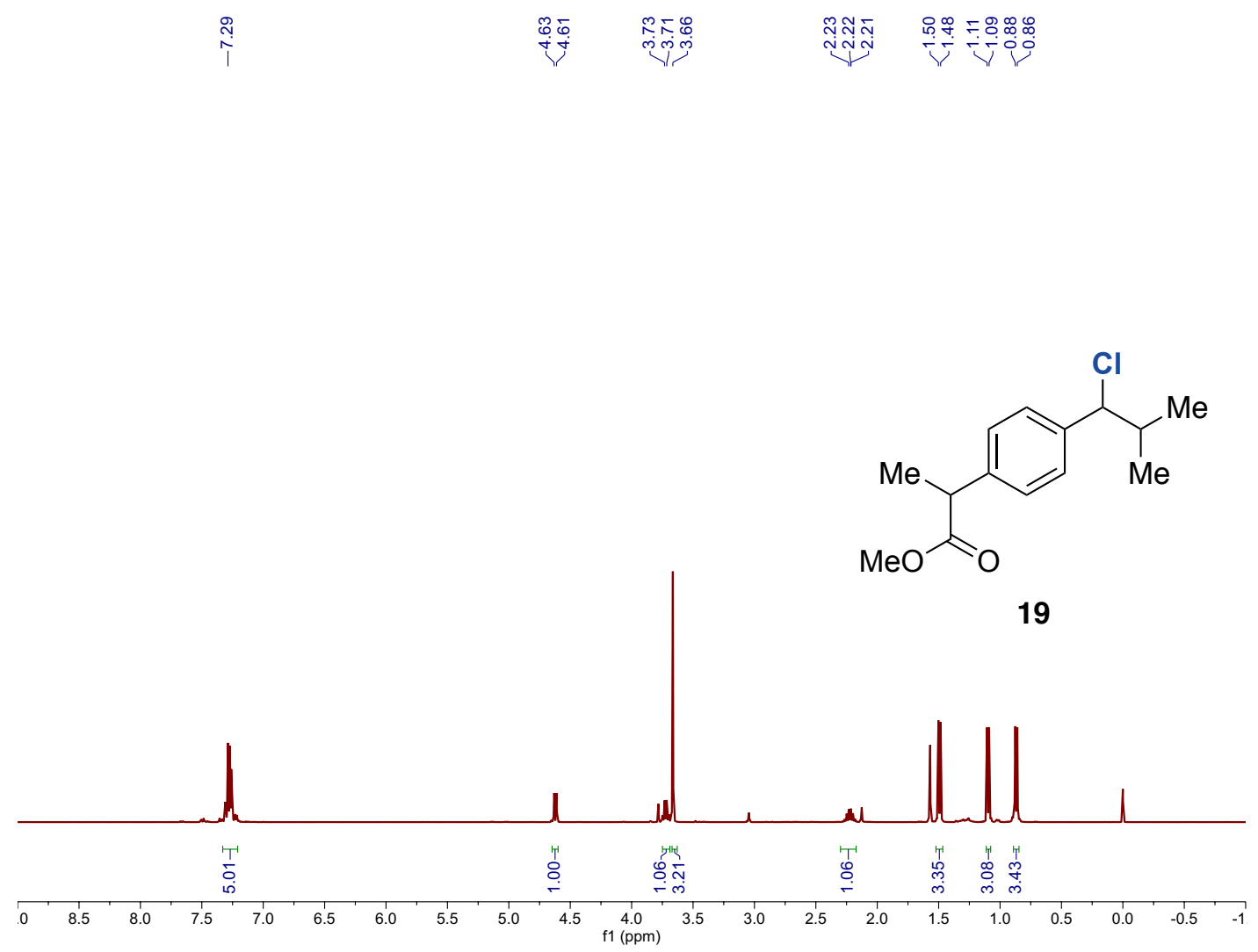

${ }^{13}$ C NMR $\left(101 \mathrm{MHz}, \mathrm{CDCl}_{3}\right)$ of 19

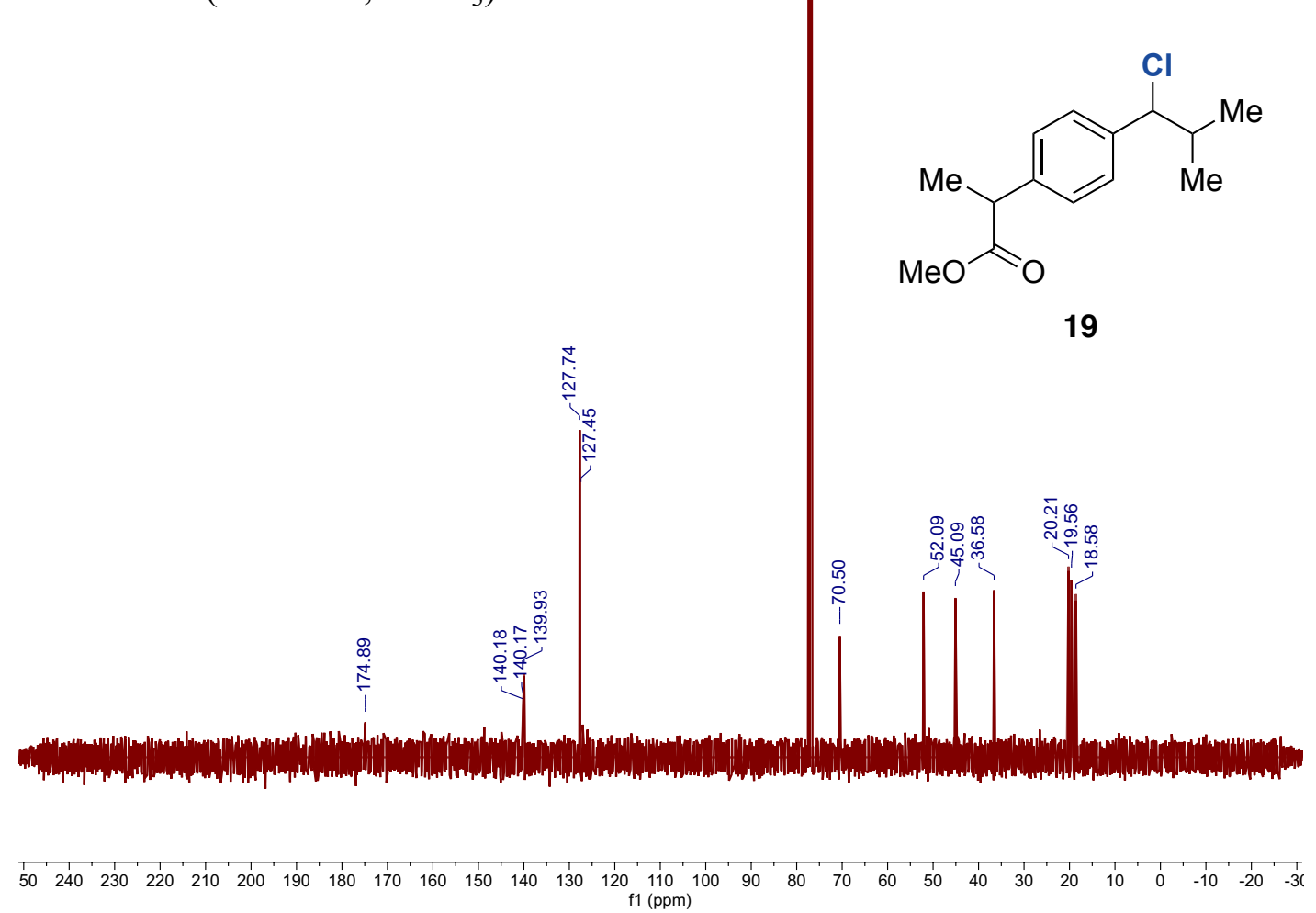


${ }^{1} \mathbf{H}$ NMR $\left(500 \mathrm{MHz}, \mathrm{CDCl}_{3}\right)$ of $\mathbf{2 0}$

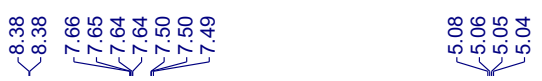

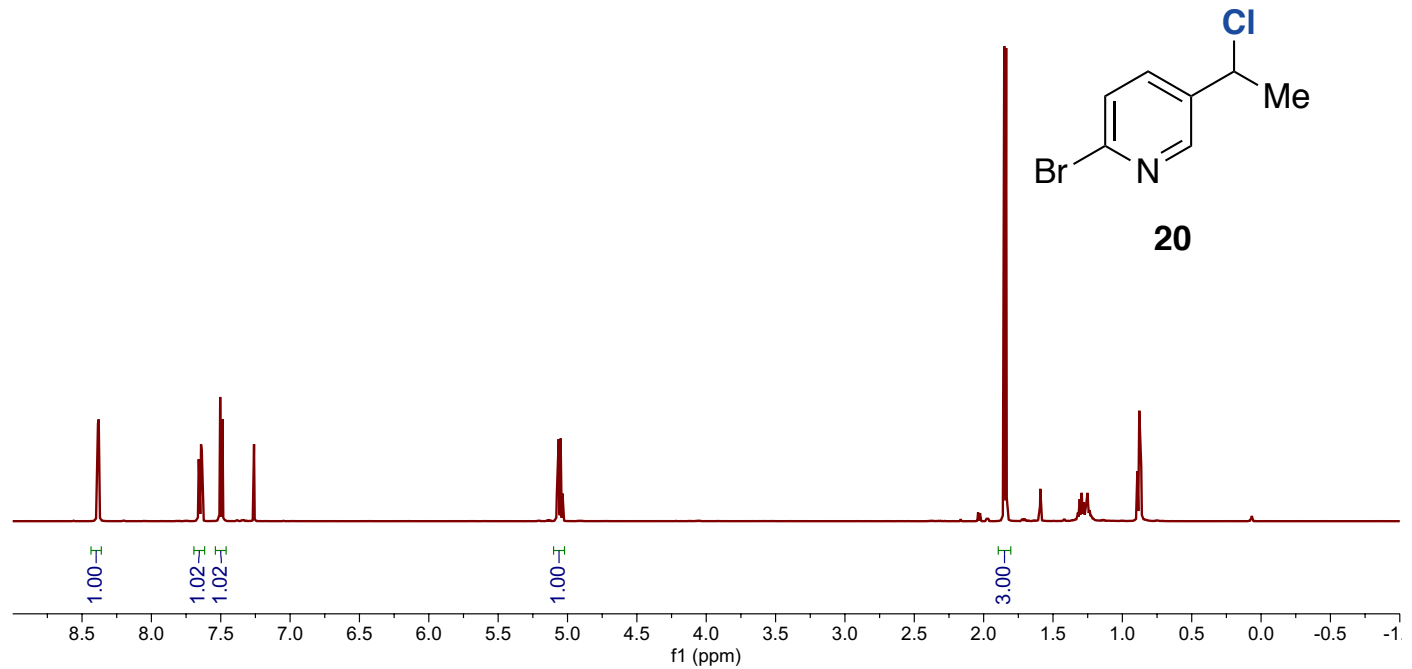

${ }^{13} \mathrm{C}$ NMR $\left(126 \mathrm{MHz}, \mathrm{CDCl}_{3}\right)$ of $\mathbf{2 0}$

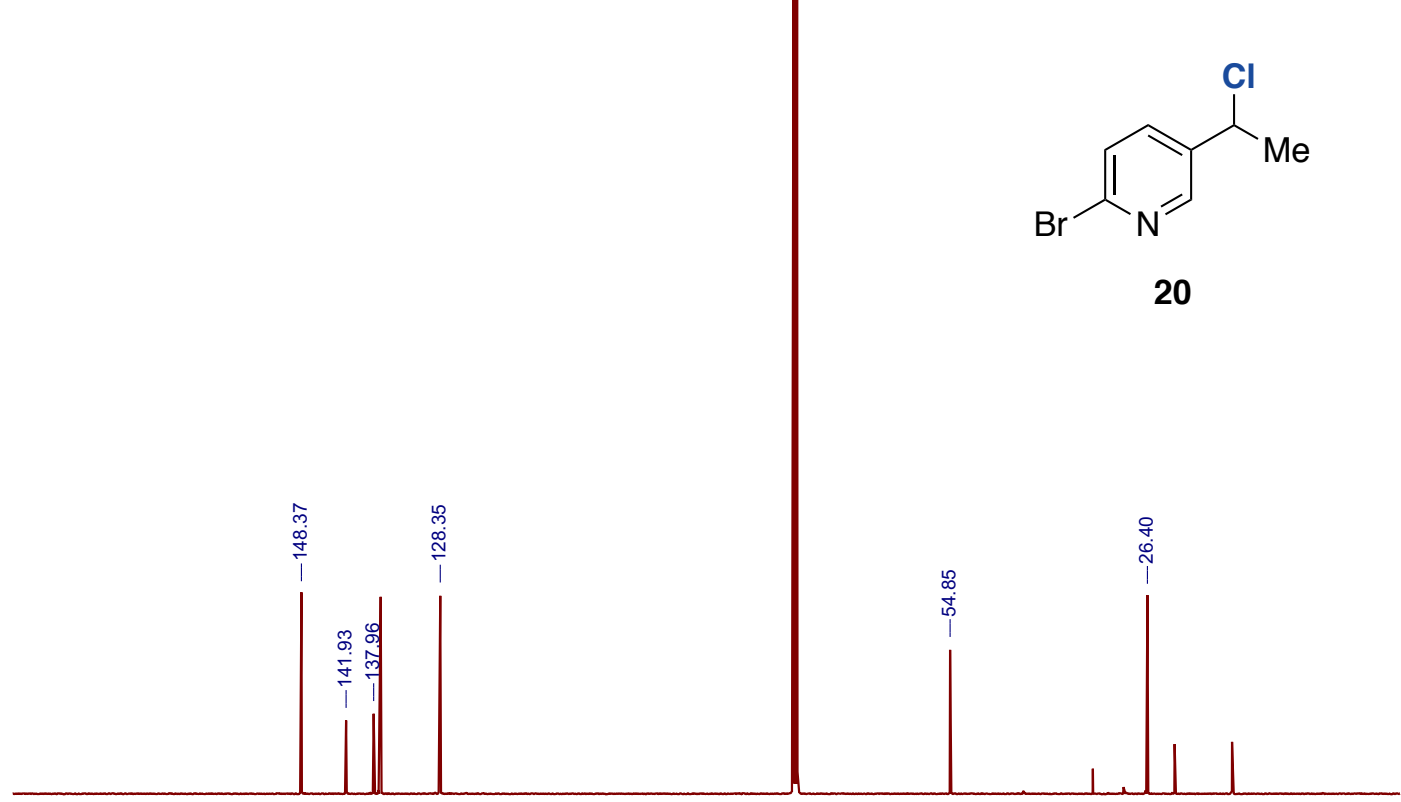

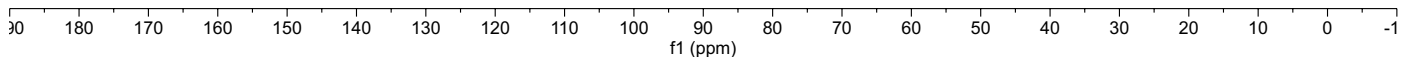


${ }^{1} \mathbf{H}$ NMR $\left(500 \mathrm{MHz}, \mathrm{CDCl}_{3}\right)$ of $\mathbf{2 1}$

i.

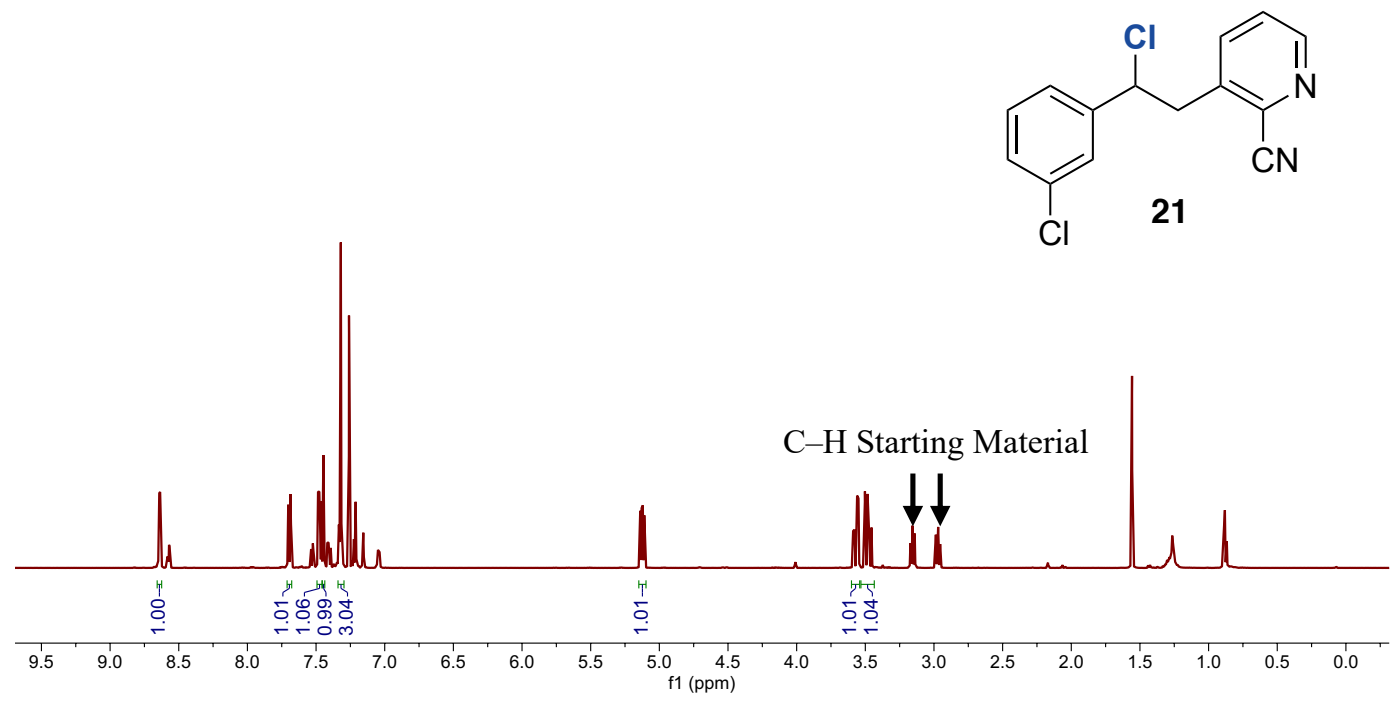

${ }^{13}$ C NMR $\left(126 \mathrm{MHz}, \mathrm{CDCl}_{3}\right)$ of 21

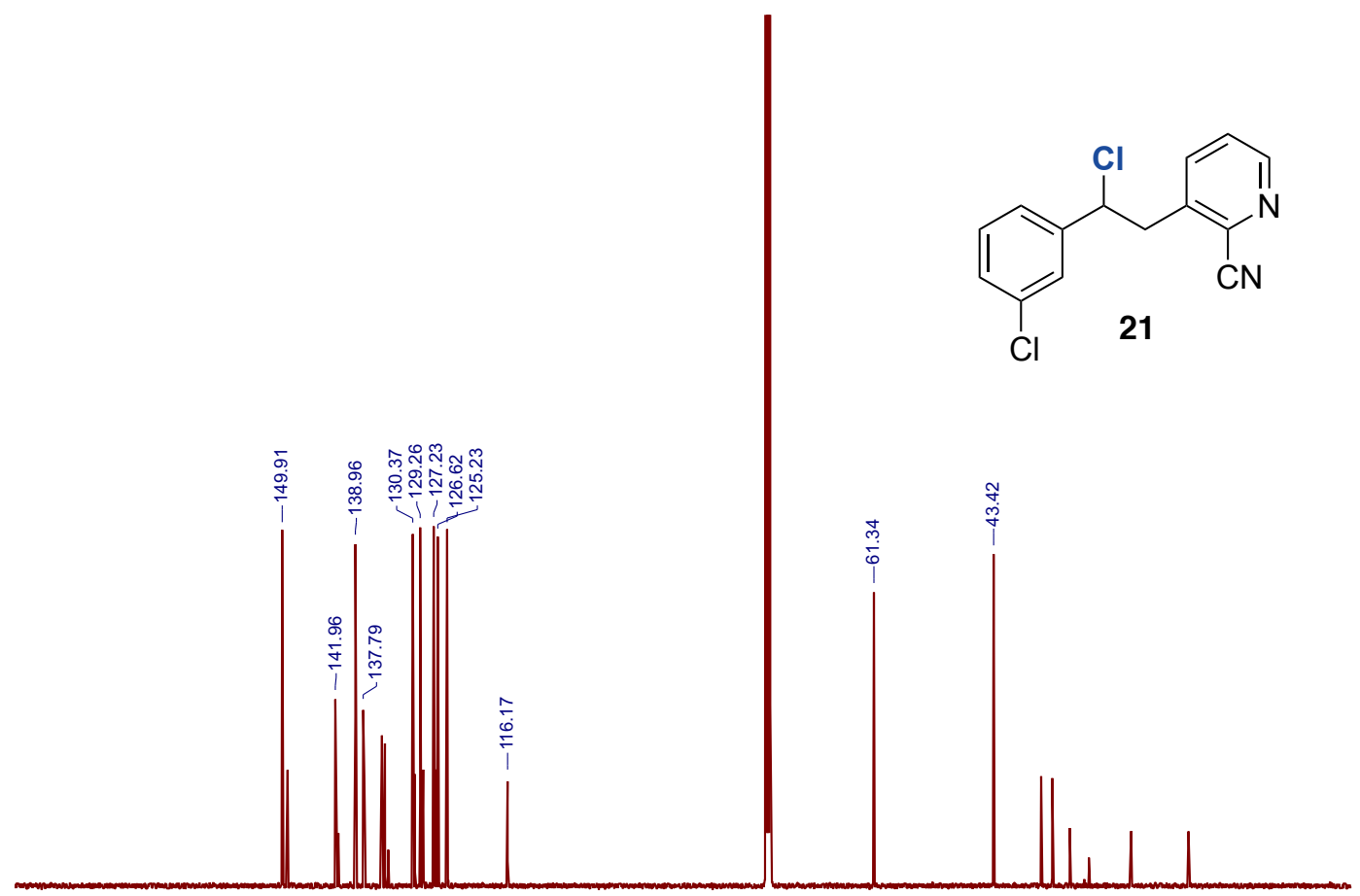

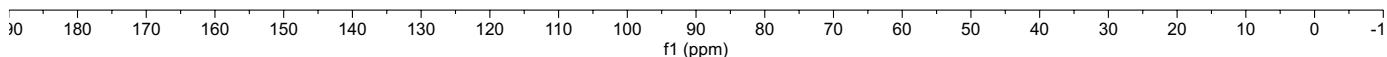


${ }^{1} \mathbf{H}$ NMR $\left(500 \mathrm{MHz}, \mathrm{CDCl}_{3}\right)$ of 22

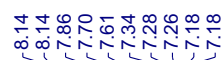

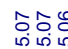

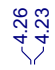

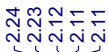

$4<$

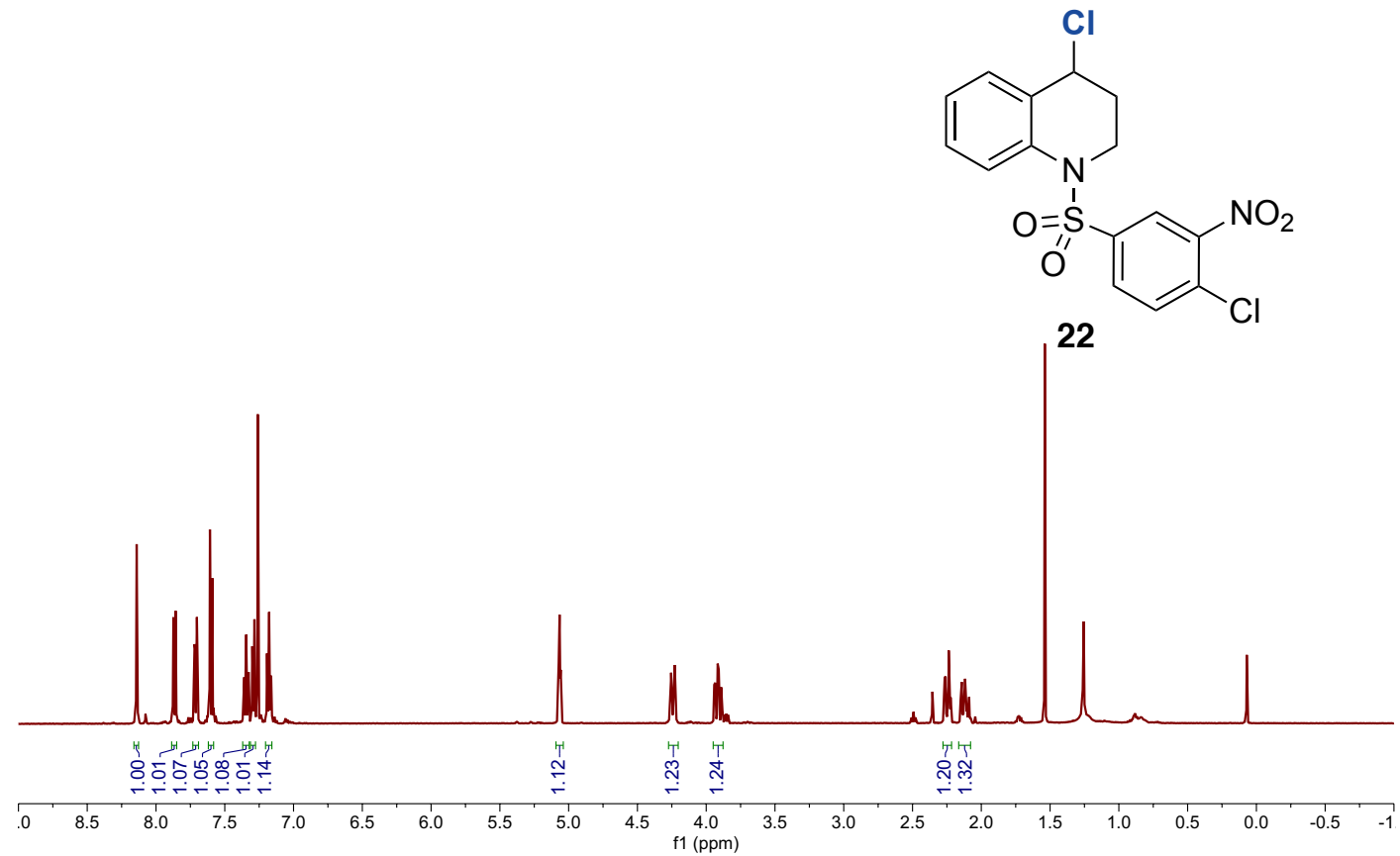

${ }^{13} \mathbf{C ~ N M R}\left(126 \mathrm{MHz}, \mathrm{CDCl}_{3}\right)$ of $\mathbf{2 2}$

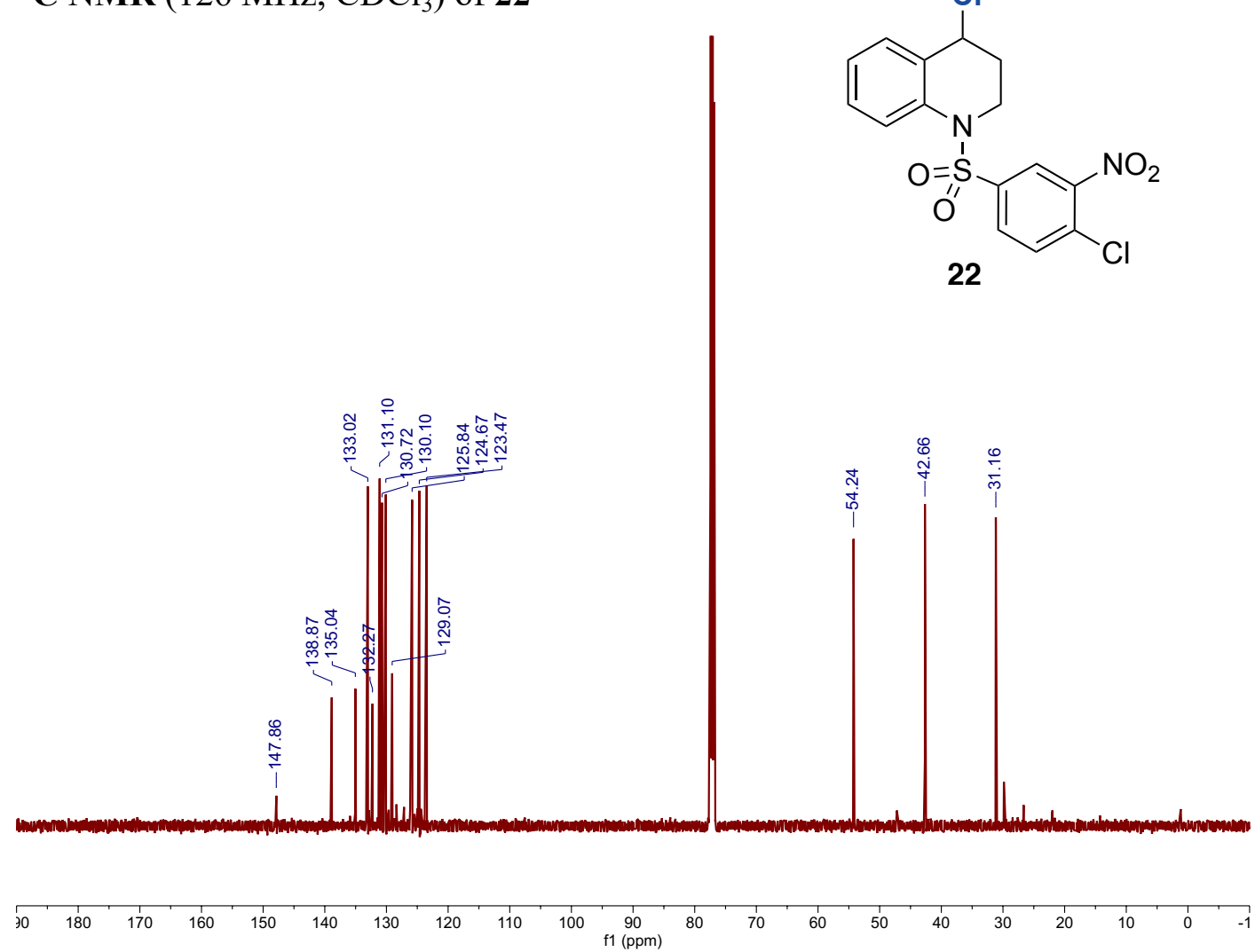


${ }^{1} \mathbf{H}$ NMR $\left(500 \mathrm{MHz}, \mathrm{CDCl}_{3}\right)$ of $\mathbf{2 3}$

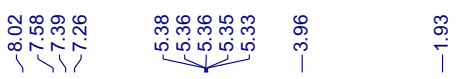

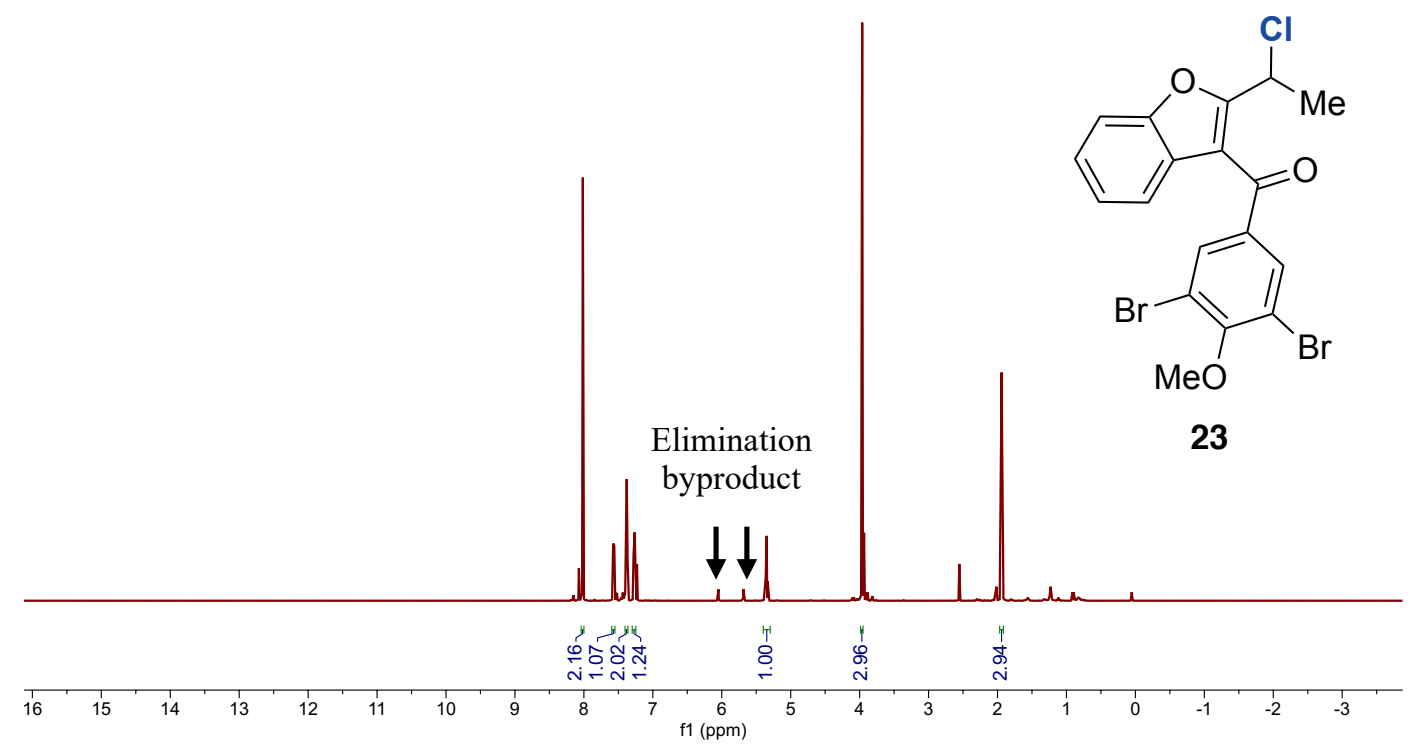

${ }^{13}$ C NMR (126 MHz, $\left.\mathrm{CDCl}_{3}\right)$ of $\mathbf{2 3}$
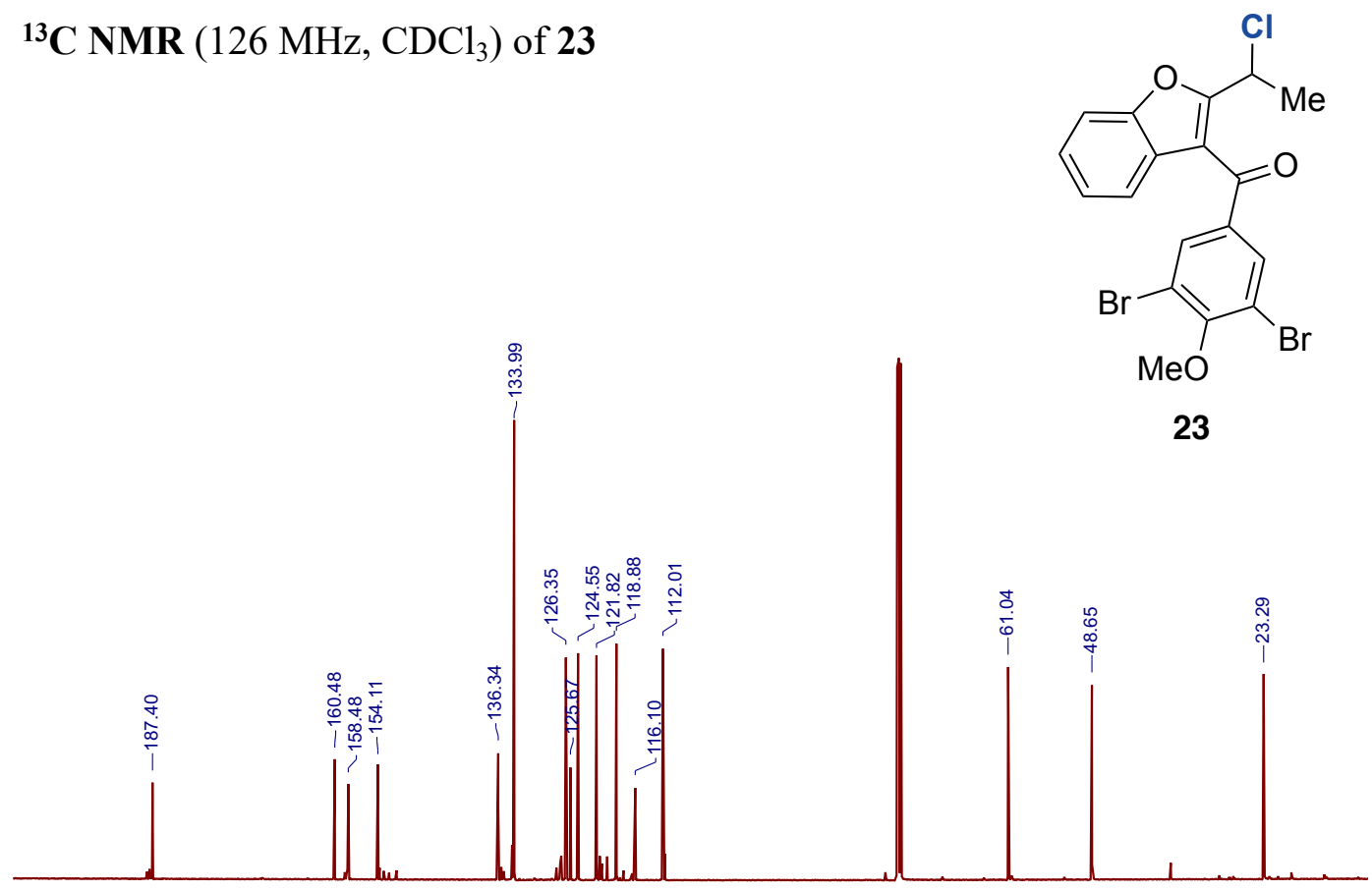

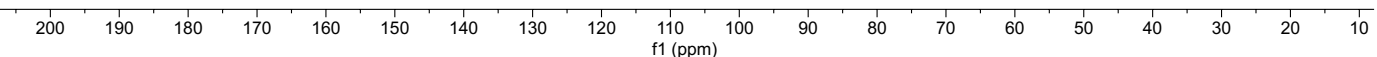


${ }^{\mathbf{1}} \mathbf{H}$ NMR $\left(500 \mathrm{MHz}, \mathrm{CDCl}_{3}\right)$ of $\mathbf{2 4}$

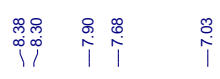

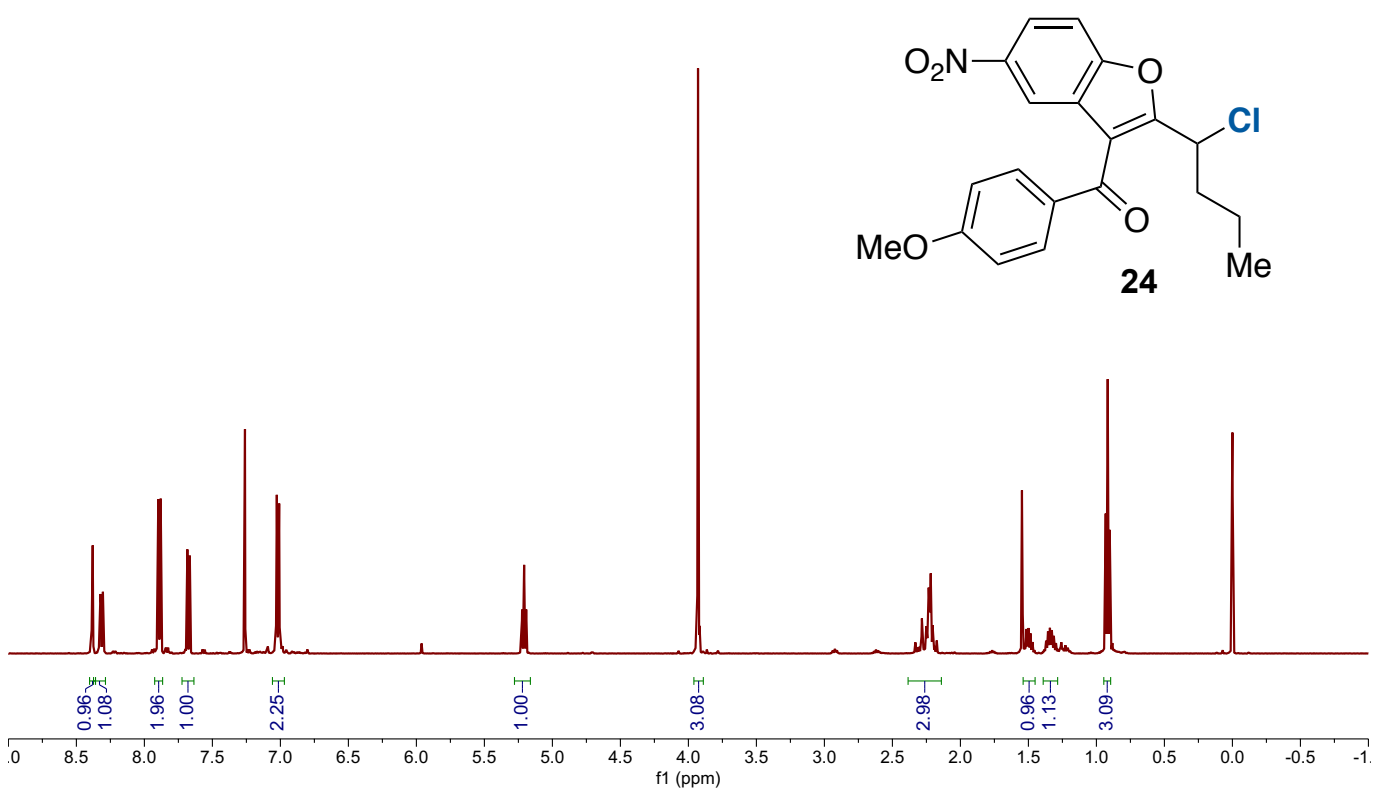

${ }^{13} \mathrm{C}$ NMR $\left(126 \mathrm{MHz}, \mathrm{CDCl}_{3}\right)$ of $\mathbf{2 4}$

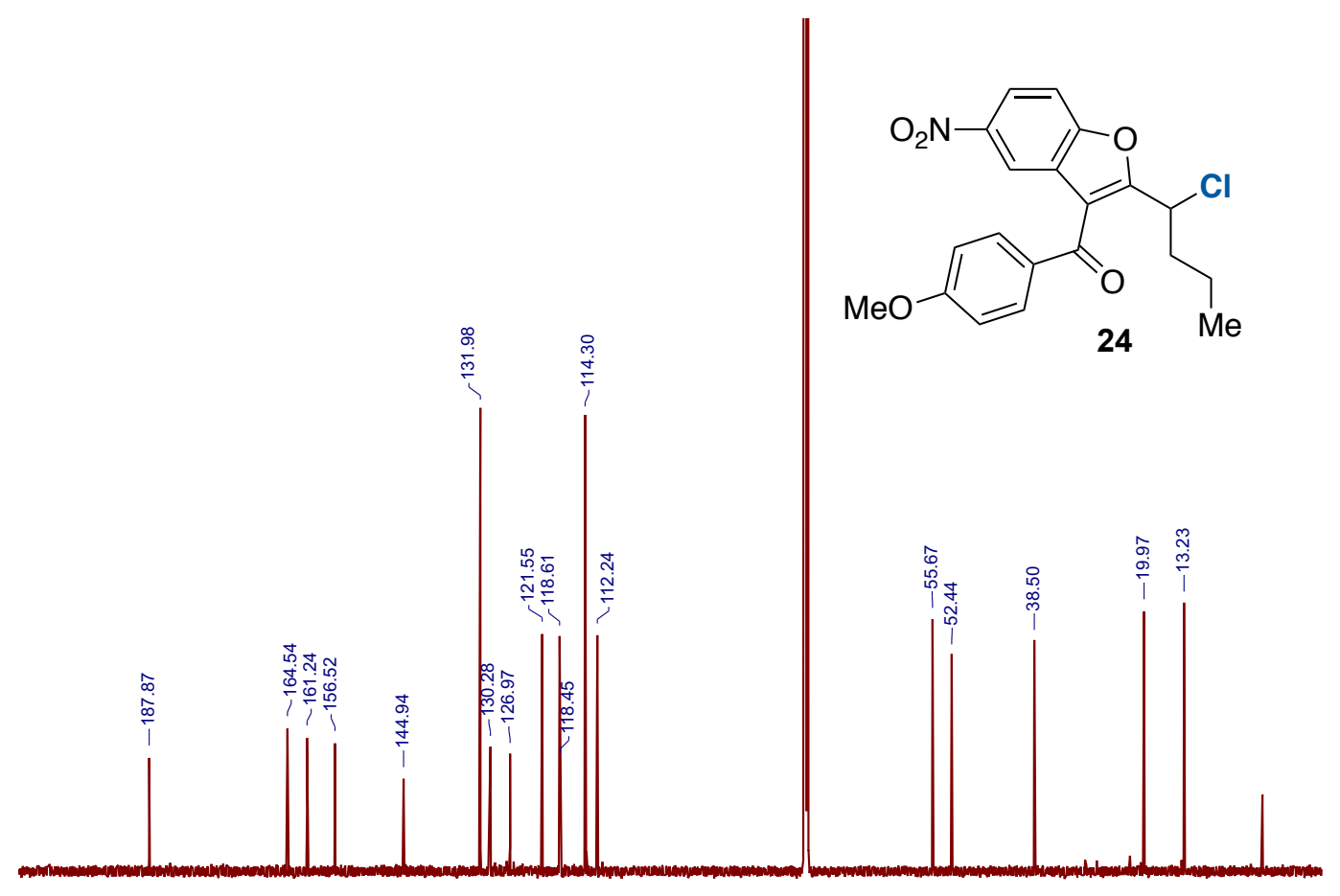

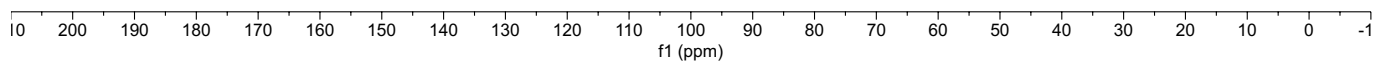


${ }^{\mathbf{1}} \mathbf{H}$ NMR $\left(500 \mathrm{MHz}, \mathrm{CDCl}_{3}\right)$ of $\mathbf{2 5}$

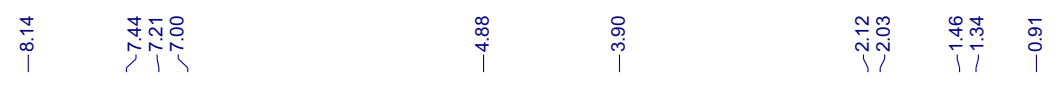

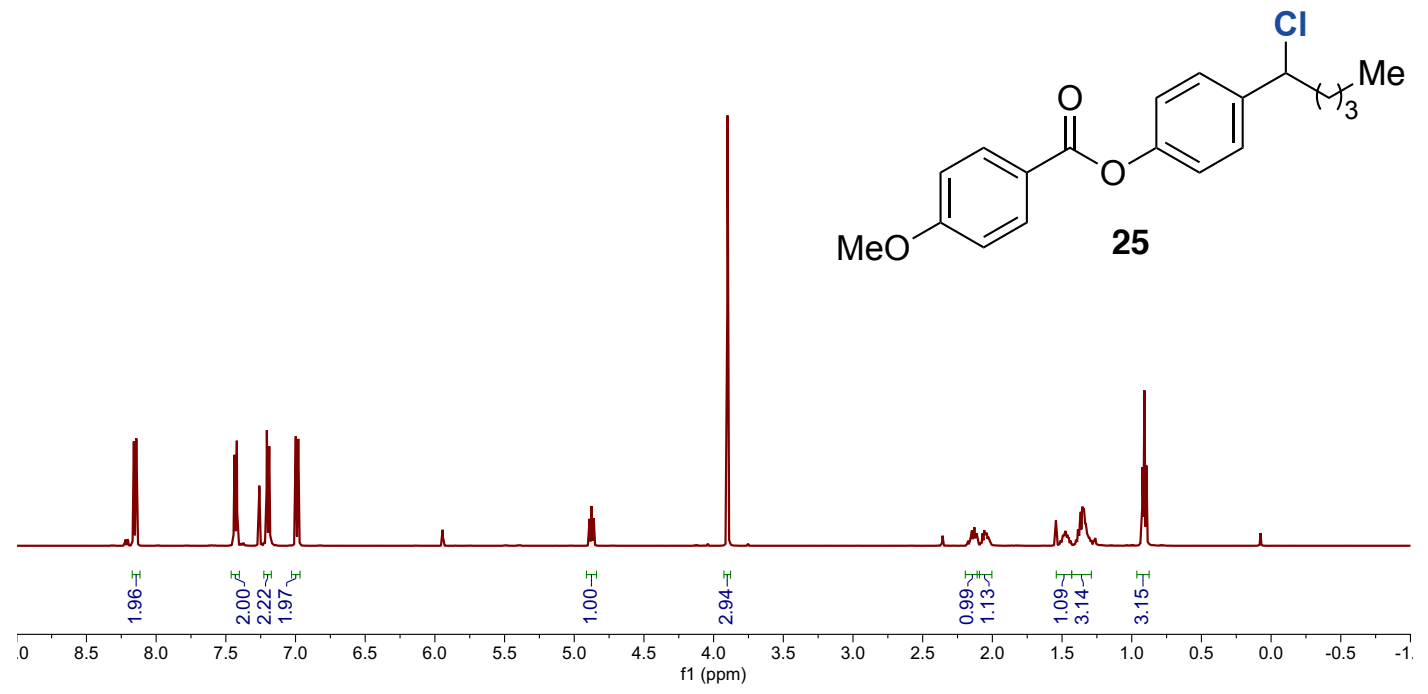

${ }^{13} \mathrm{C}$ NMR $\left(126 \mathrm{MHz}, \mathrm{CDCl}_{3}\right)$ of 25

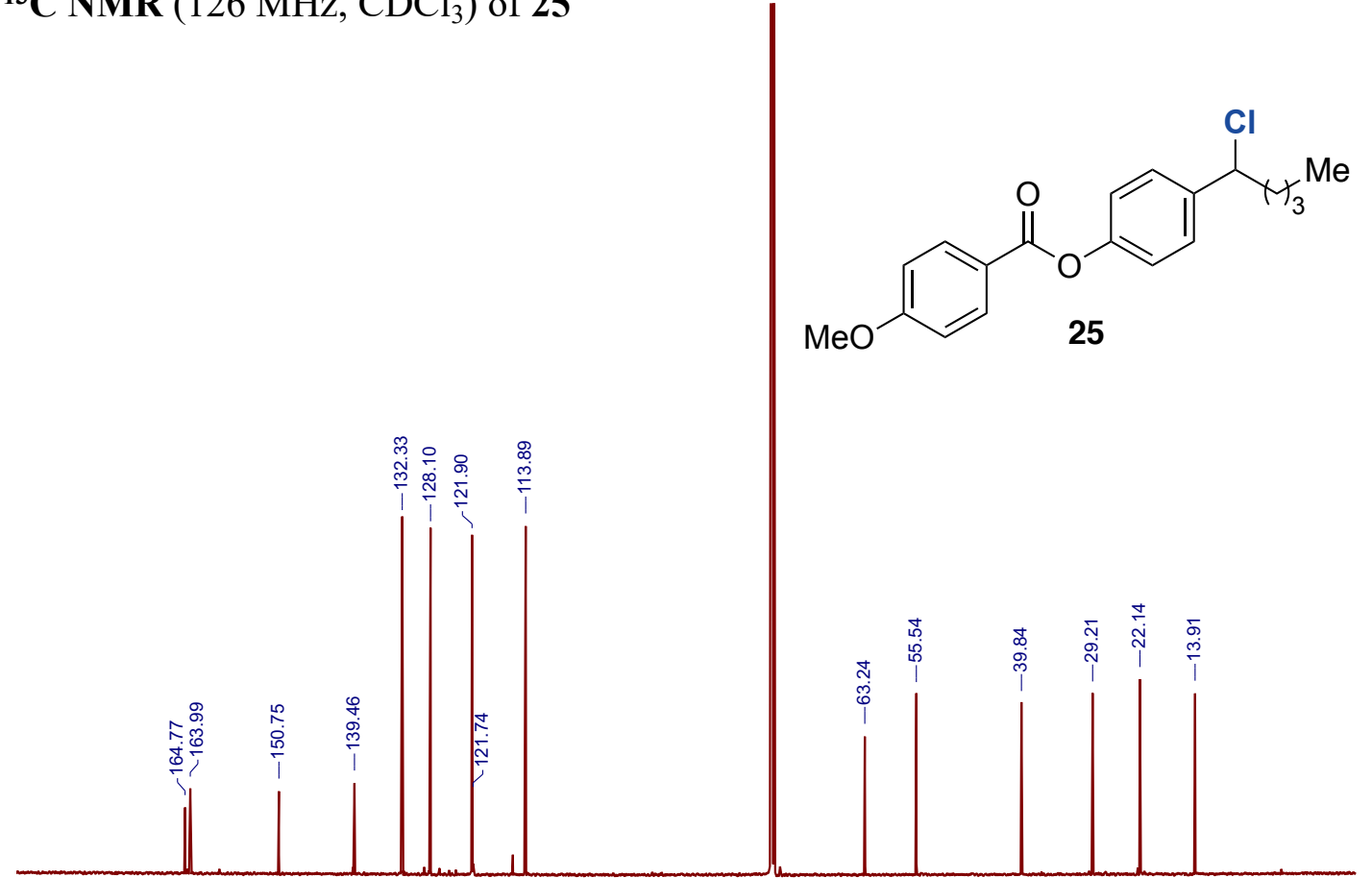

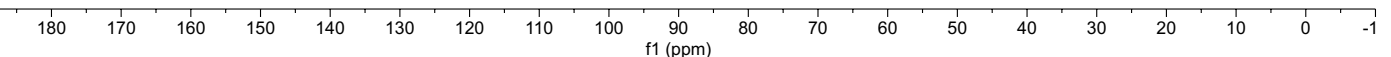


${ }^{\mathbf{1}} \mathbf{H}$ NMR $\left(400 \mathrm{MHz}, \mathrm{CDCl}_{3}\right)$ of $\mathbf{2 6}$

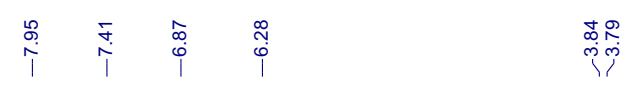

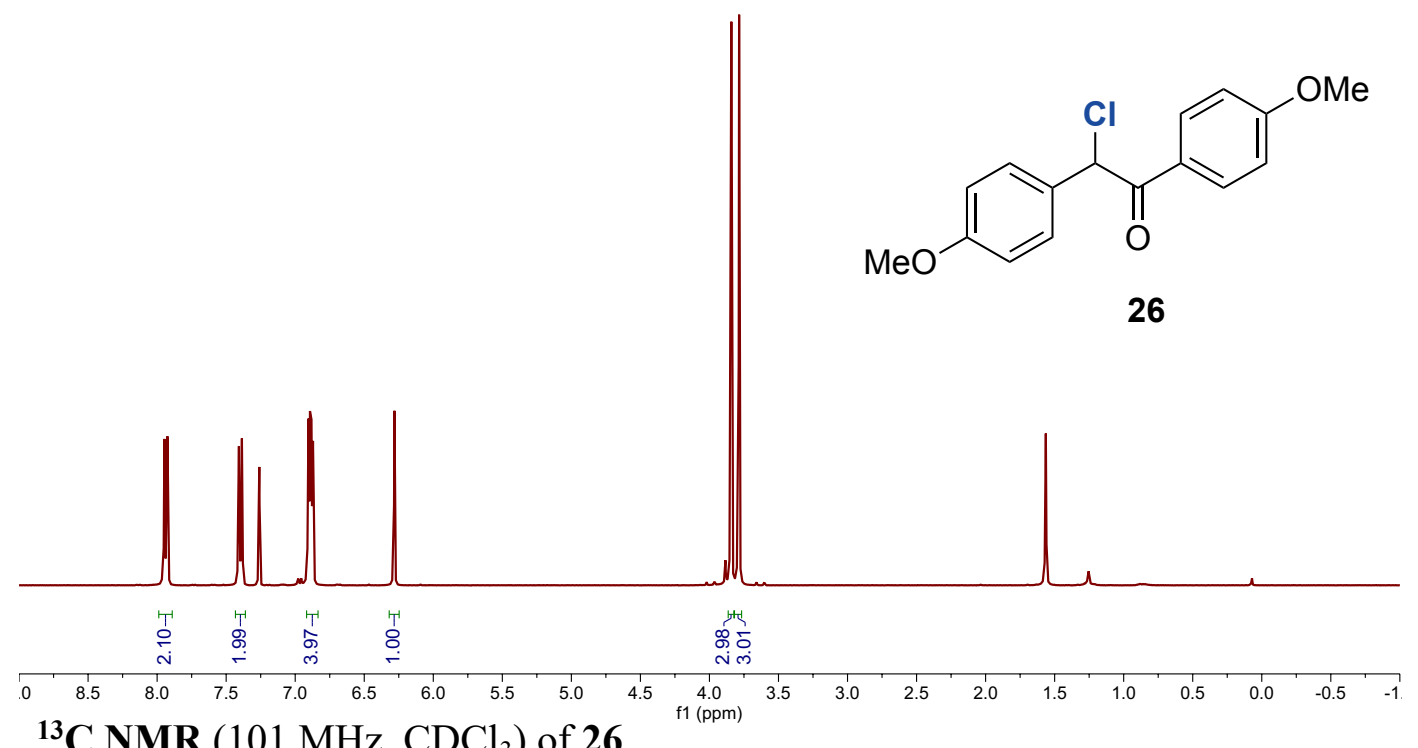

${ }^{13} \mathbf{C}$ NMR $\left(101 \mathrm{MHz}, \mathrm{CDCl}_{3}\right)$ of $\mathbf{2 6}$

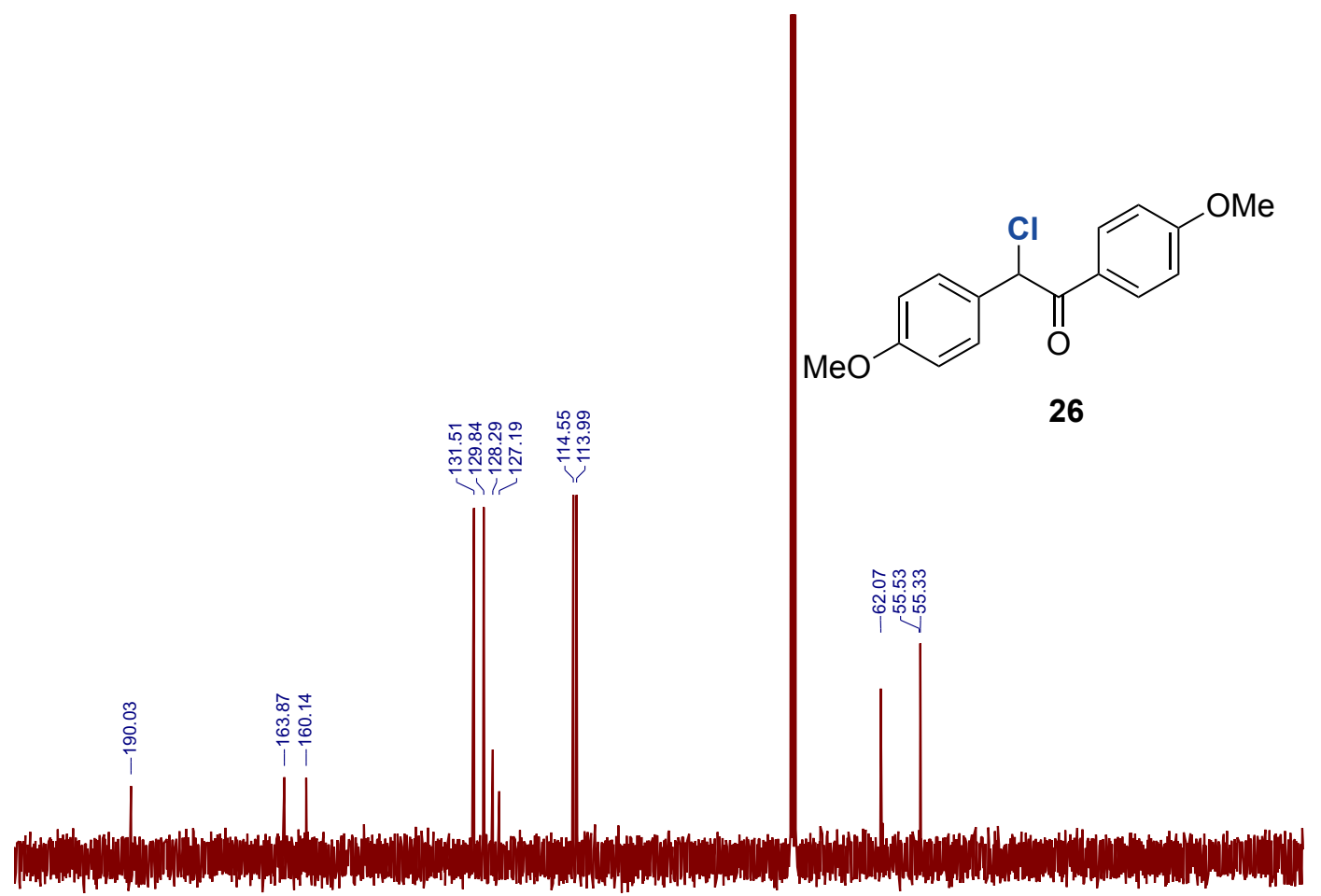

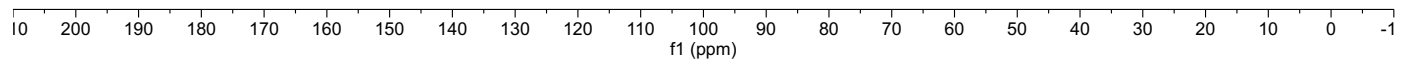


${ }^{1} \mathbf{H}$ NMR $\left(500 \mathrm{MHz}, \mathrm{CDCl}_{3}\right)$ of 27

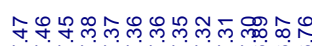

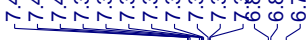

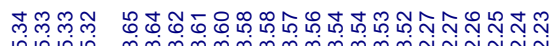

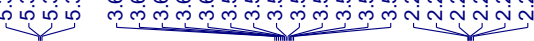

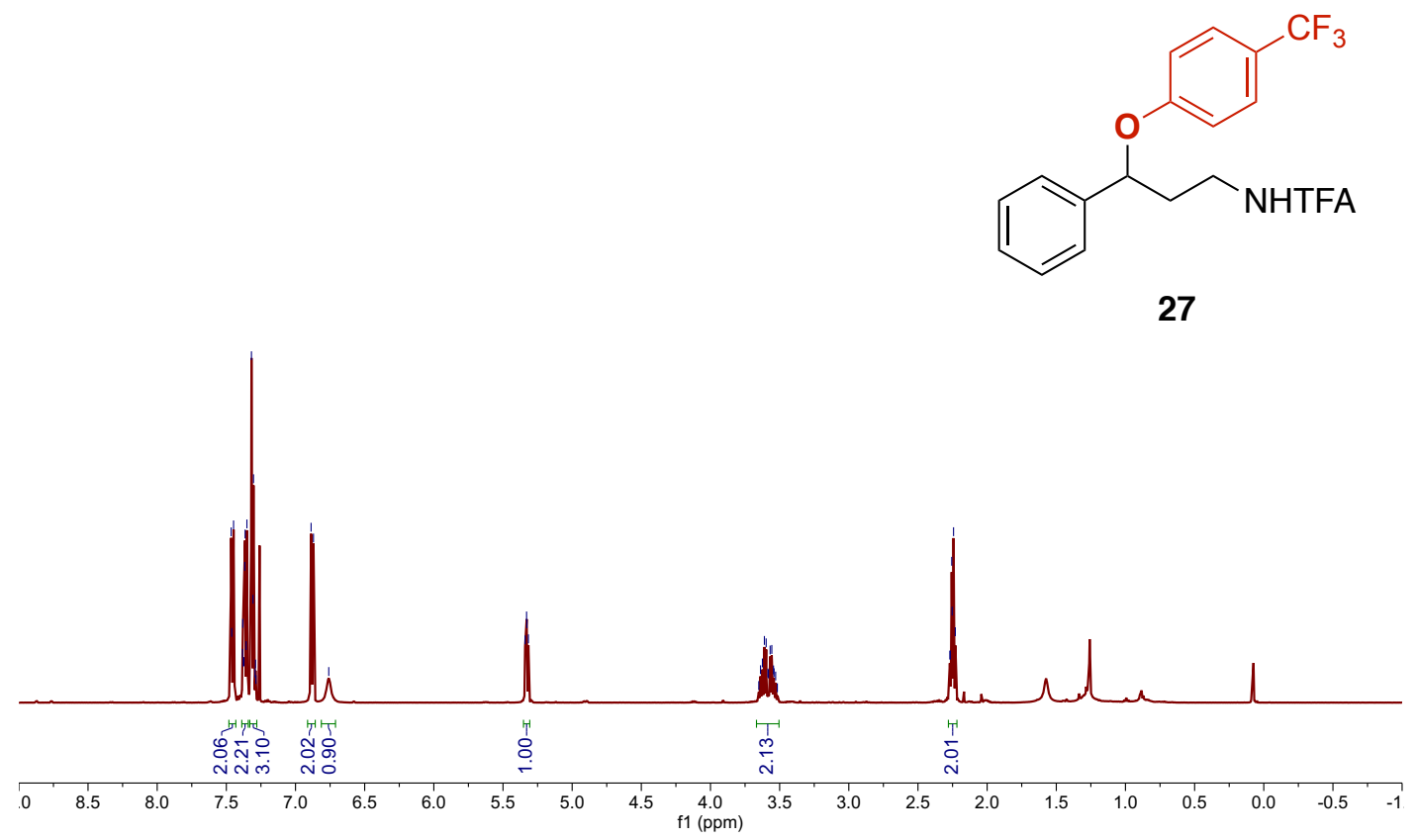

${ }^{13}$ C NMR $\left(126 \mathrm{MHz}, \mathrm{CDCl}_{3}\right)$ of $\mathbf{2 8}$

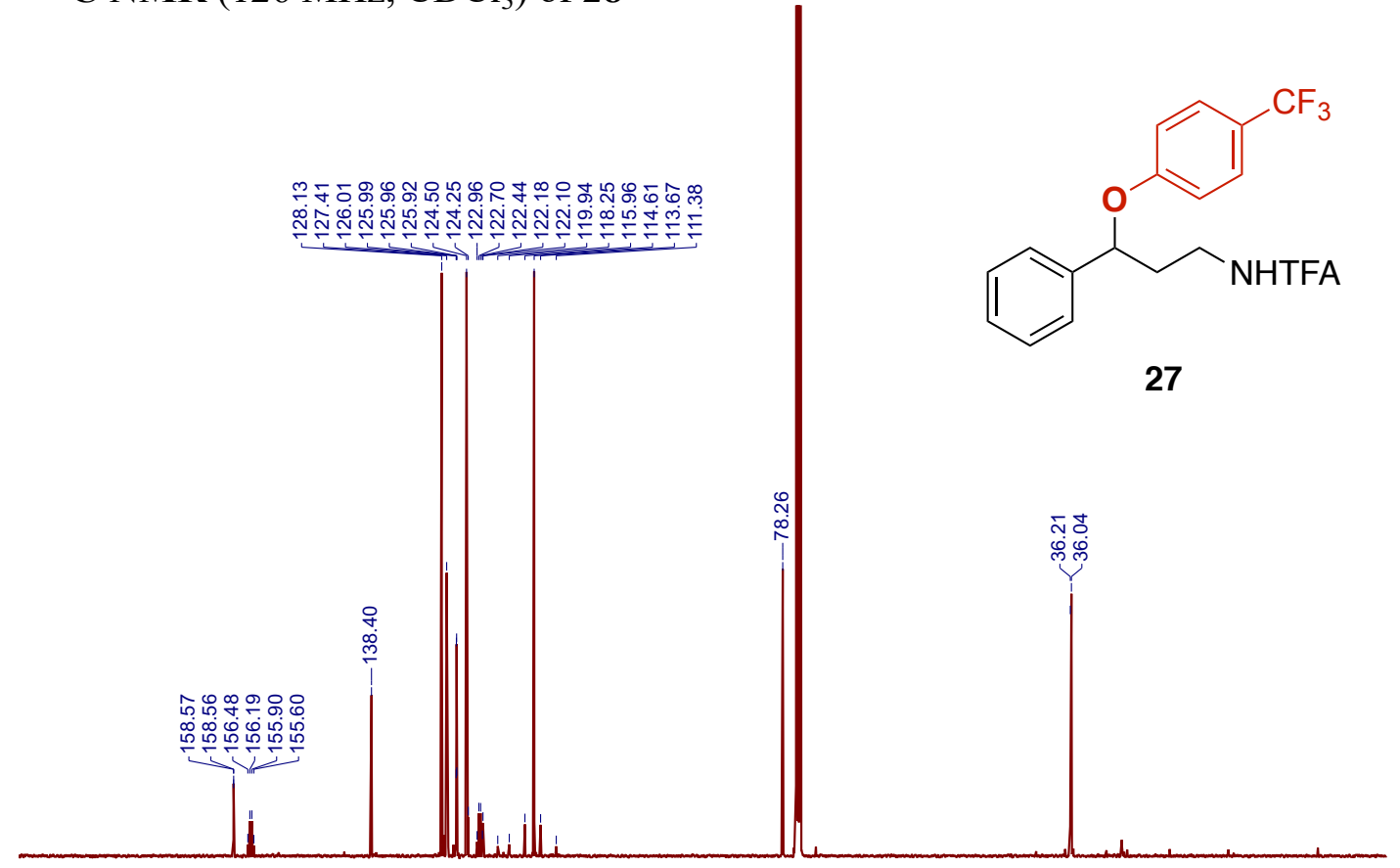


${ }^{19}$ F NMR $\left(377 \mathrm{MHz}, \mathrm{CDCl}_{3}\right)$ of 27

$\begin{array}{ll}\bar{r} & \overline{0} \\ \overline{\dot{j}} & \stackrel{p}{i} \\ i & i\end{array}$

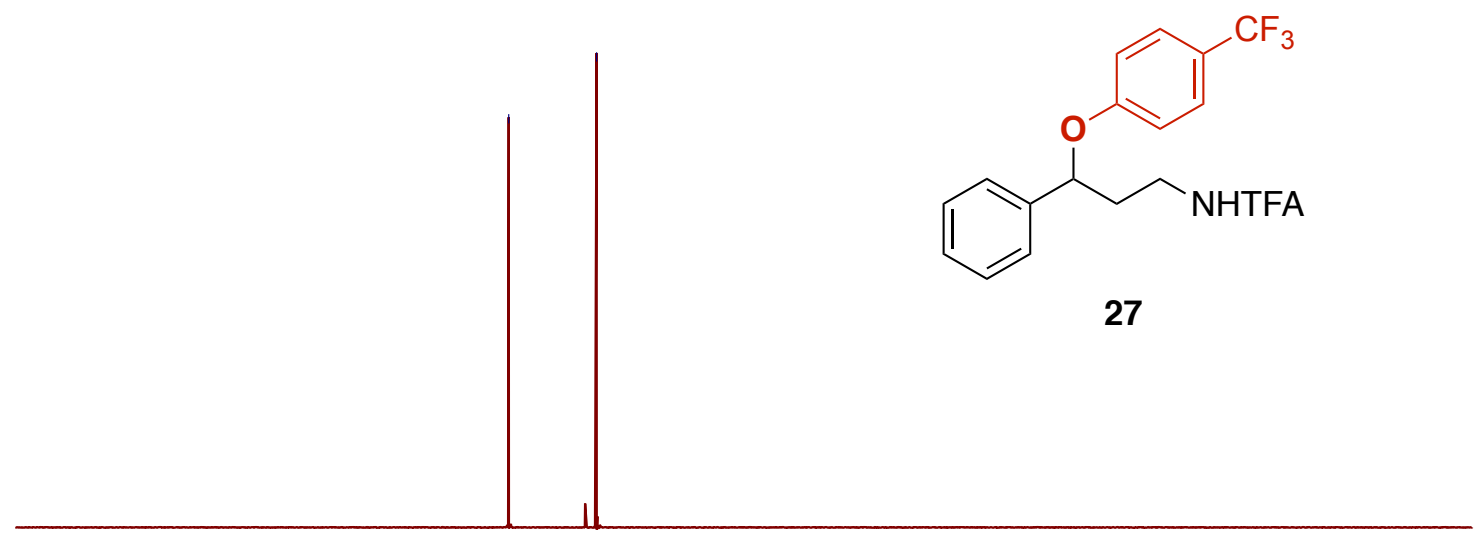

$\begin{array}{llllllllllllllllllllllll}10 & 0 & -10 & -20 & -30 & -40 & -50 & -60 & -70 & -80 & -90 & -100 & -110 & -120 & -130 & -140 & -150 & -160 & -170 & -180 & -190 & -200 & -210 & 1\end{array}$ 
${ }^{\mathbf{1}} \mathbf{H}$ NMR $\left(500 \mathrm{MHz}, \mathrm{CDCl}_{3}\right)$ of $\mathbf{2 8}$

苾不

武证然

வุํำ

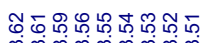

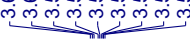

$\sqrt[\text { กิ }]{\text { กิ }}$<smiles>FC(F)NCCC(Oc1ccc(Cl)cc1)c1ccccc1</smiles>

28

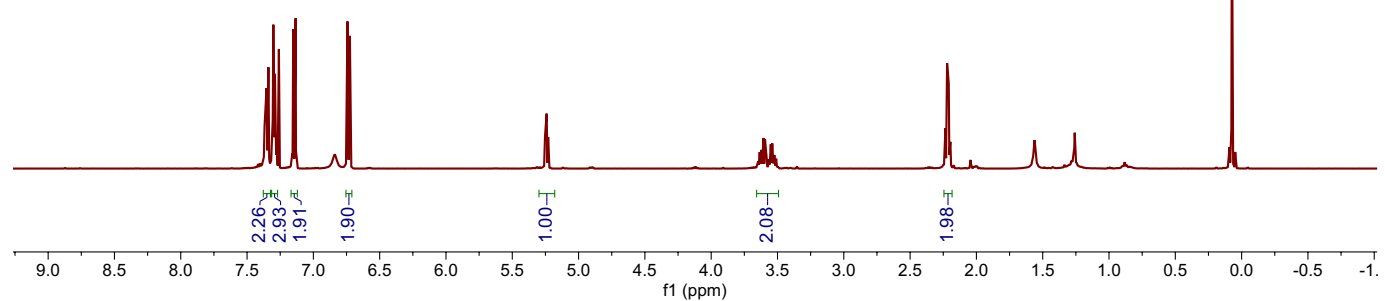

${ }^{13} \mathbf{C}$ NMR $\left(126 \mathrm{MHz}, \mathrm{CDCl}_{3}\right)$ of $\mathbf{2 8}$

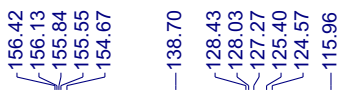

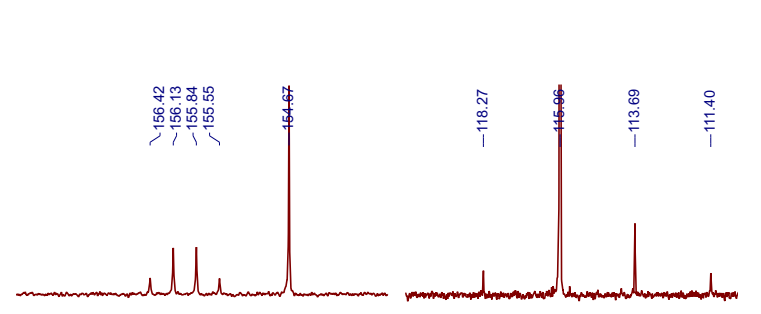

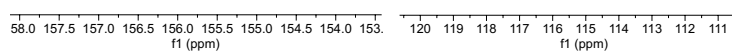

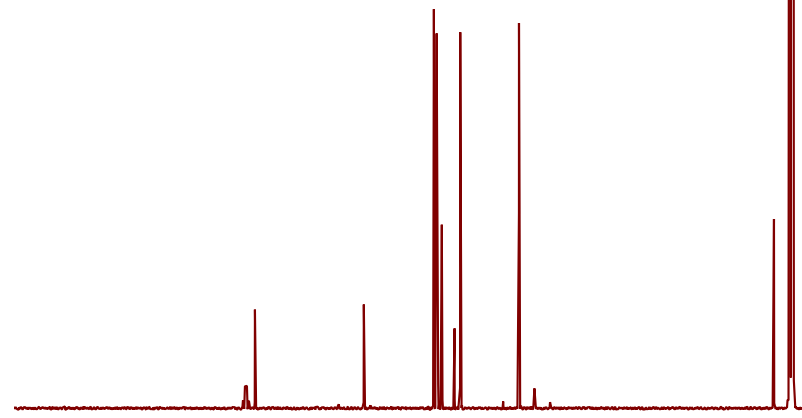<smiles>FC(F)NCCC(Oc1ccc(Cl)cc1)c1ccccc1</smiles>

28

제

jo

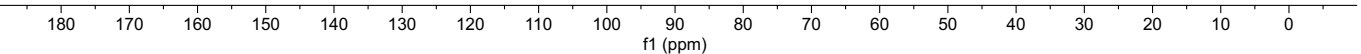


${ }^{19}$ F NMR $\left(377 \mathrm{MHz}, \mathrm{CDCl}_{3}\right)$ of $\mathbf{2 8}$

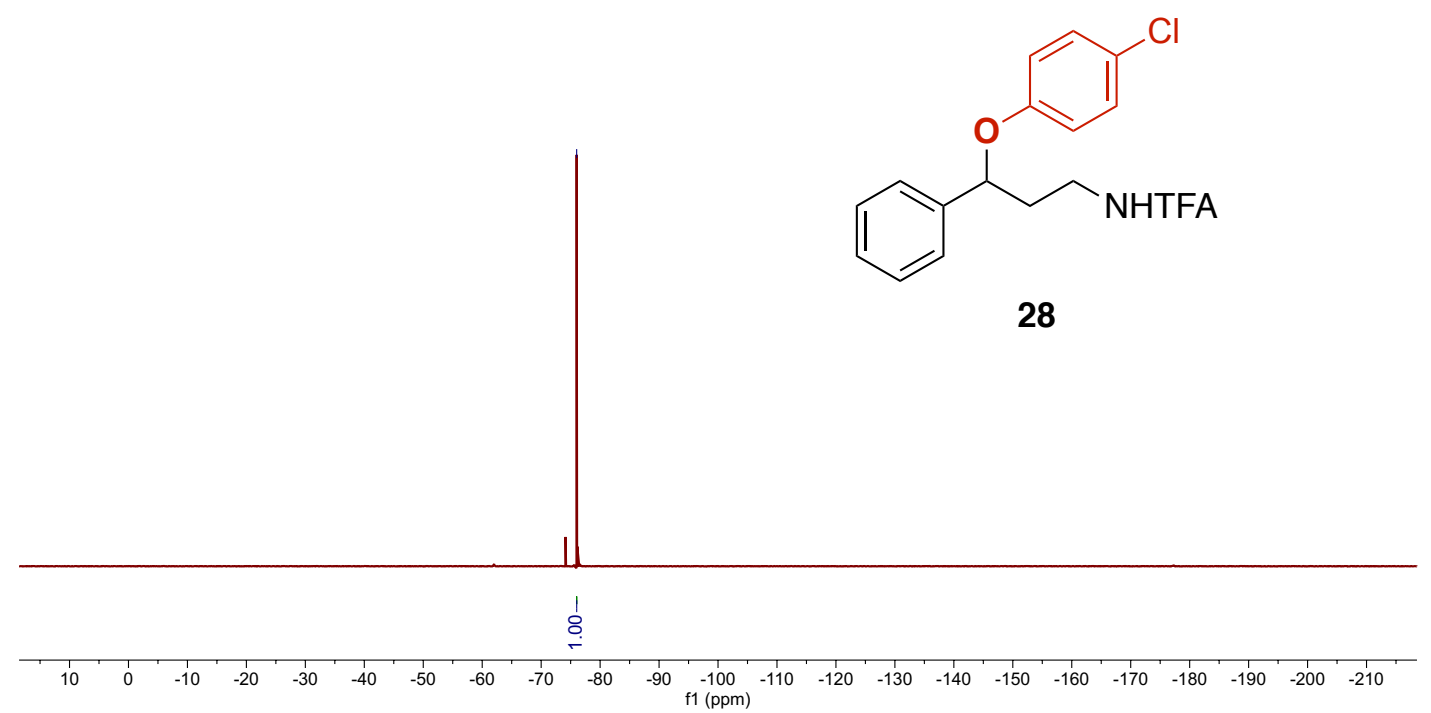


${ }^{1} \mathbf{H}$ NMR $\left(500 \mathrm{MHz}, \mathrm{CDCl}_{3}\right)$ of 29

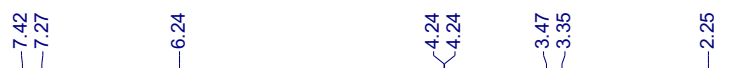

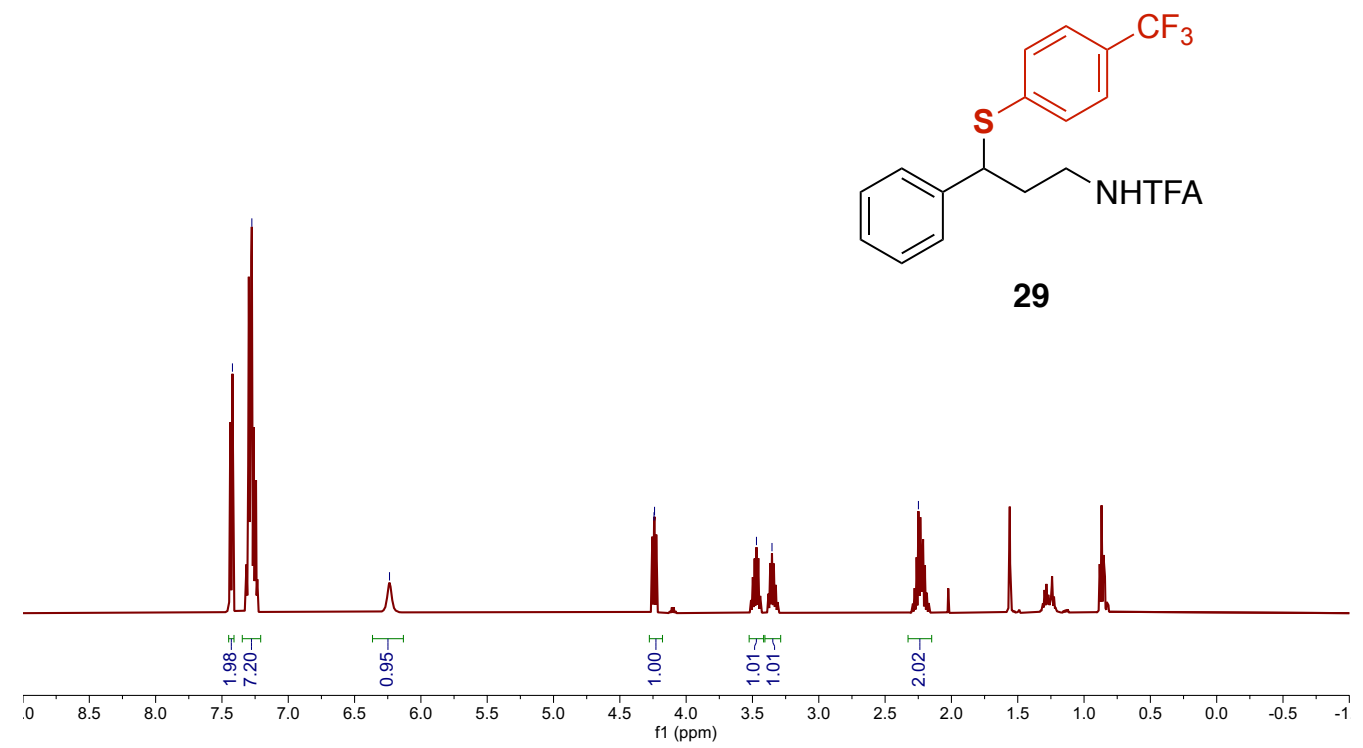

${ }^{13} \mathbf{C}$ NMR $\left(126 \mathrm{MHz}, \mathrm{CDCl}_{3}\right)$ of 29

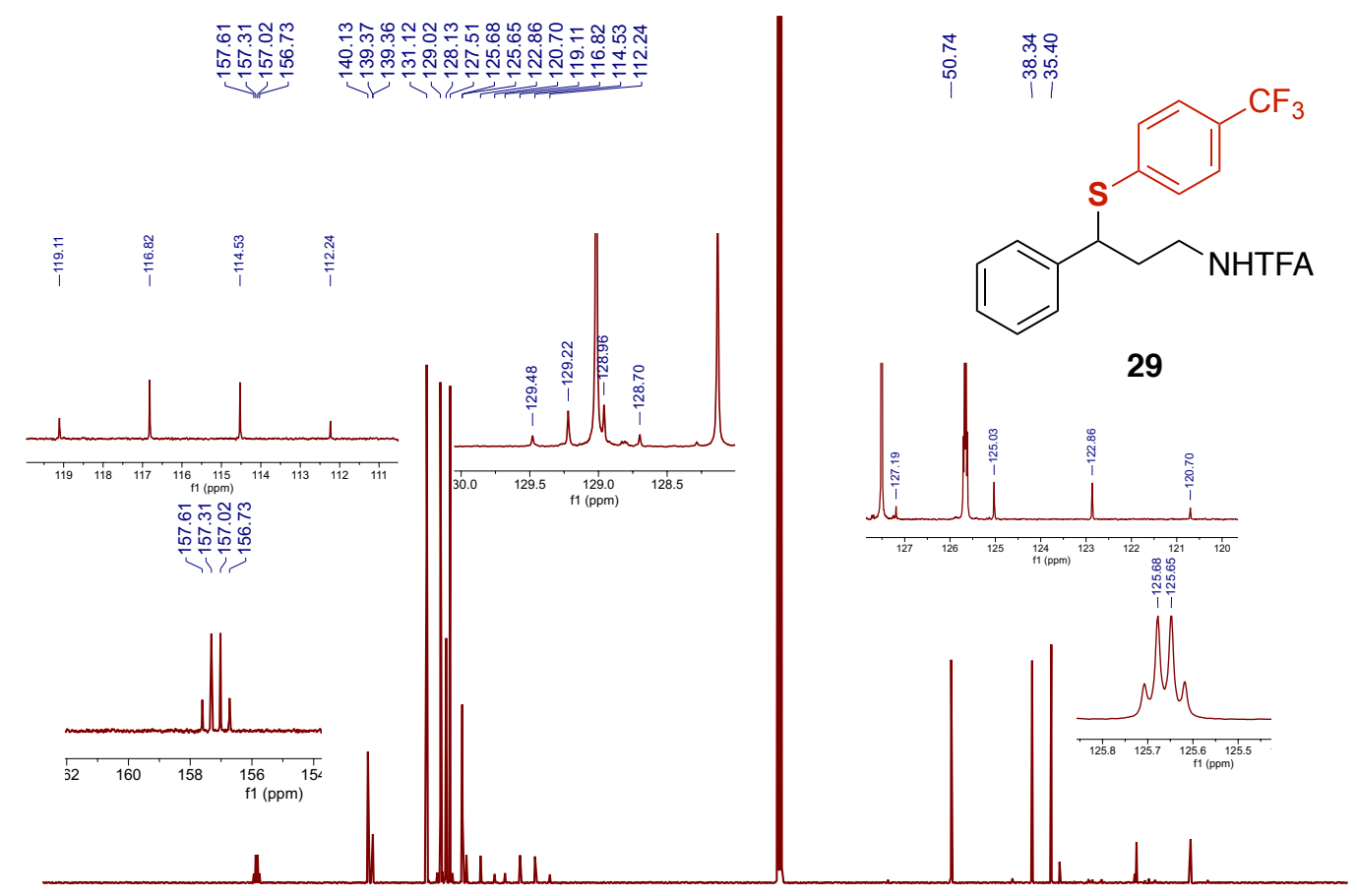

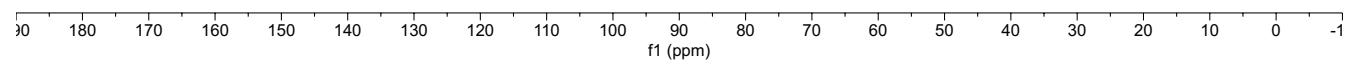


${ }^{19}$ F NMR $\left(377 \mathrm{MHz}, \mathrm{CDCl}_{3}\right)$ of 29

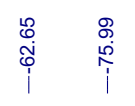

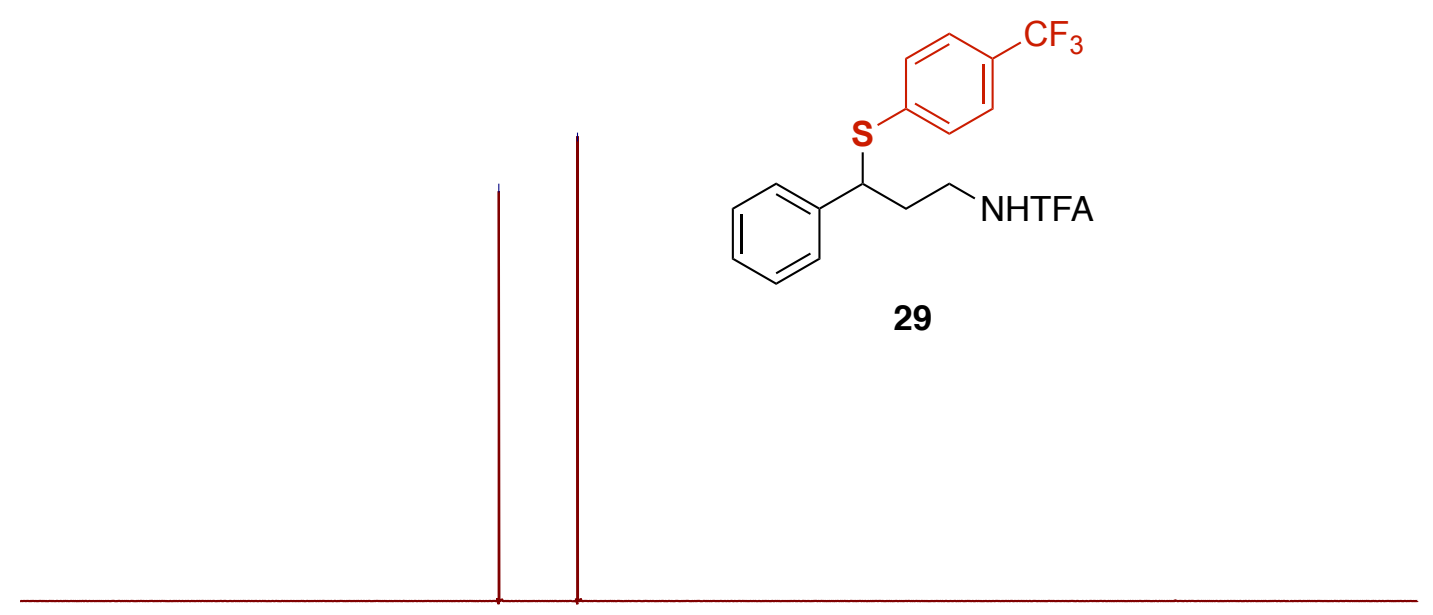

\begin{tabular}{lllllllllllllllllllllll}
\hline 10 & 0 & -10 & -20 & -30 & -40 & -50 & -60 & -70 & -80 & -90 & -100 & -110 & -120 & -130 & -140 & -150 & -160 & -170 & -180 & -190 & -200 & -210
\end{tabular} 
${ }^{\mathbf{1}} \mathbf{H}$ NMR $\left(500 \mathrm{MHz}, \mathrm{CDCl}_{3}\right)$ of $\mathbf{3 0}$

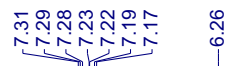

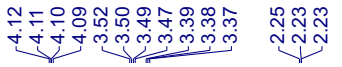

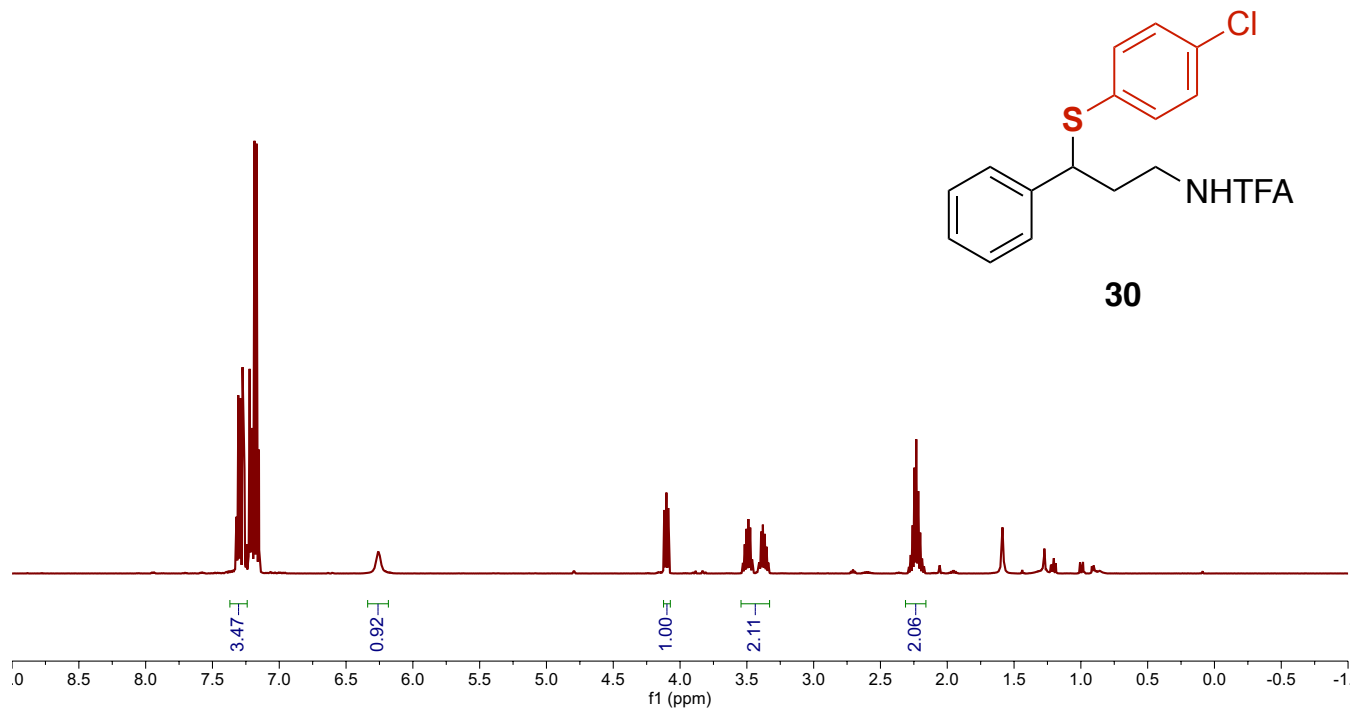

$\left.{ }^{13} \mathbf{C ~ N M R ~ ( 1 2 6 ~ M H z , ~} \mathrm{CDCl}_{3}\right)$ of $\mathbf{3 0}$

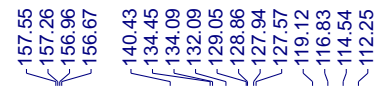

क़े

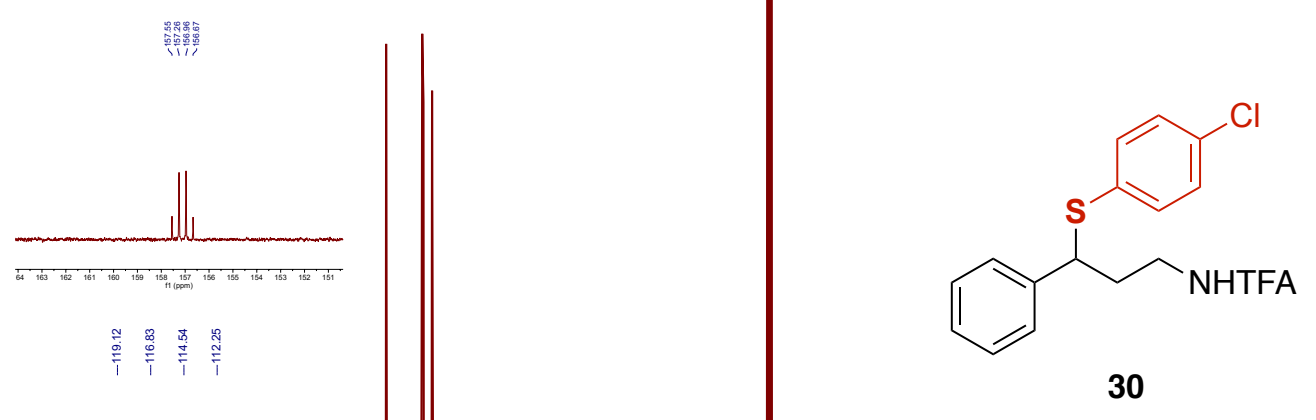

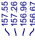
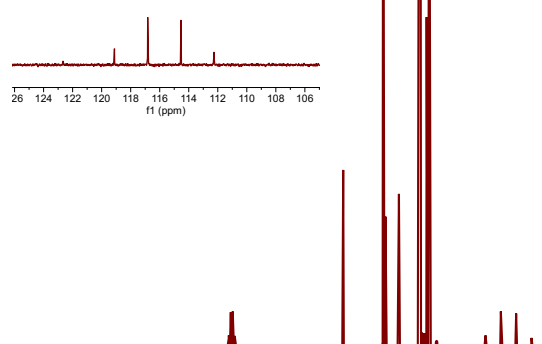

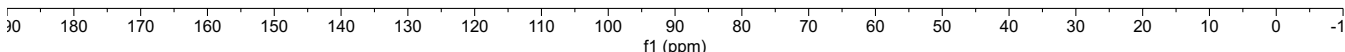


${ }^{19} \mathbf{F}$ NMR $\left(377 \mathrm{MHz}, \mathrm{CDCl}_{3}\right)$ of $\mathbf{3 0}$

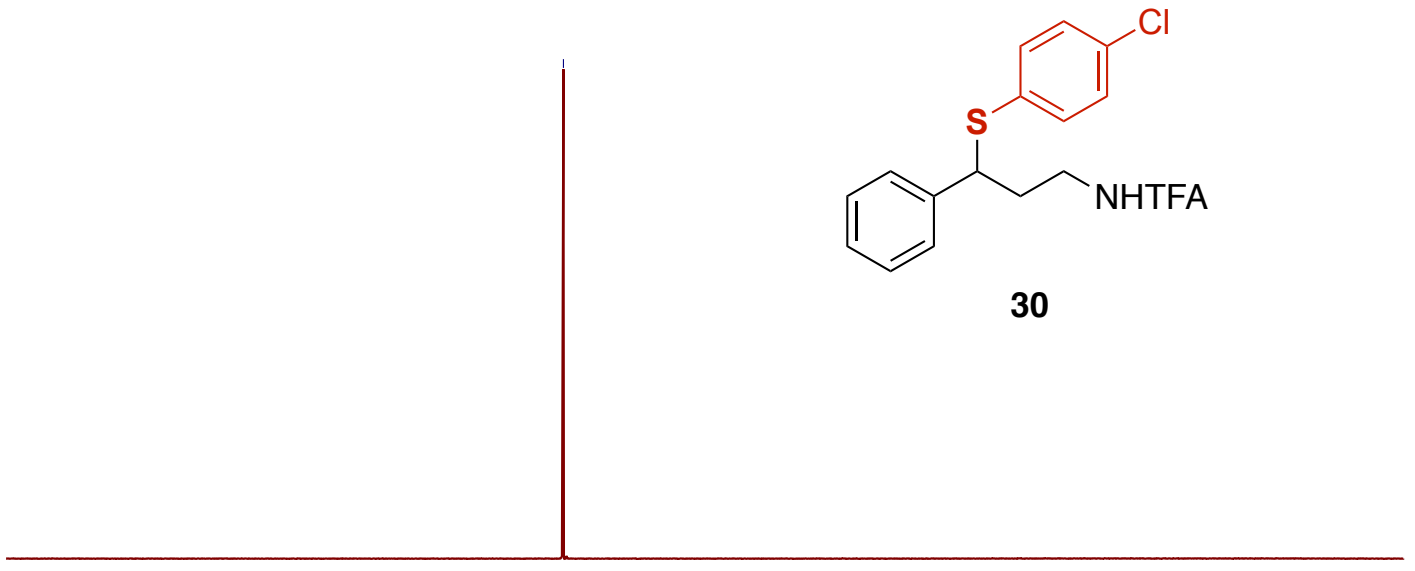

$\begin{array}{lllllllllllllllllllllllll}10 & 0 & -10 & -20 & -30 & -40 & -50 & -60 & -70 & -80 & -90 & -100 & -110 & -120 & -130 & -140 & -150 & -160 & -170 & -180 & -190 & -200 & -210\end{array}$ 
${ }^{1} \mathbf{H}$ NMR $\left(500 \mathrm{MHz}, \mathrm{CDCl}_{3}\right)$ of $\mathbf{3 1}$

$\underbrace{\infty}$

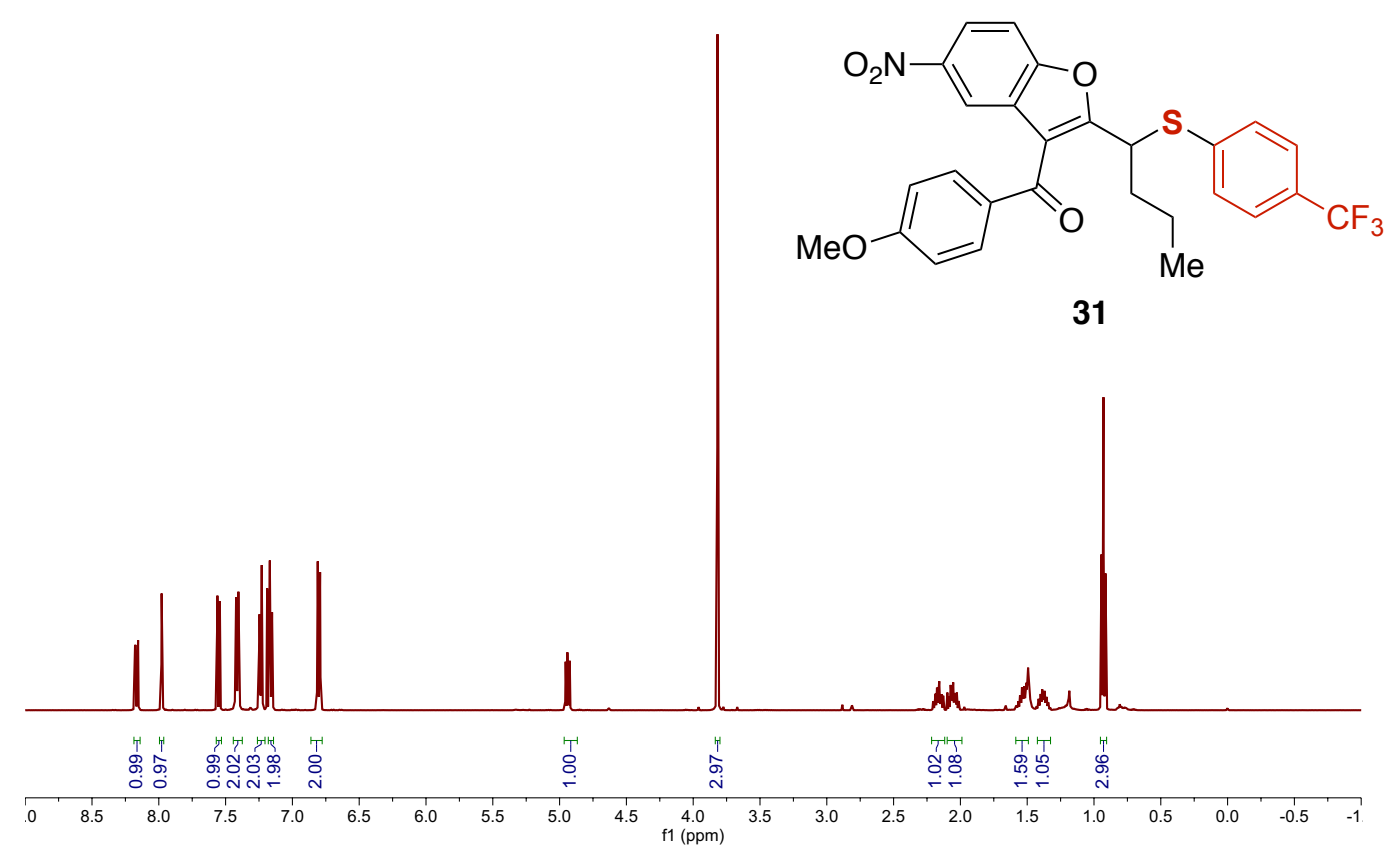

${ }^{13} \mathbf{C ~ N M R}\left(126 \mathrm{MHz}, \mathrm{CDCl}_{3}\right)$ of $\mathbf{3 1}$

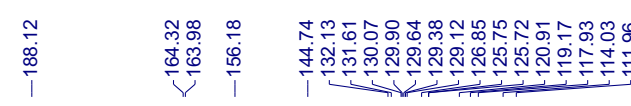
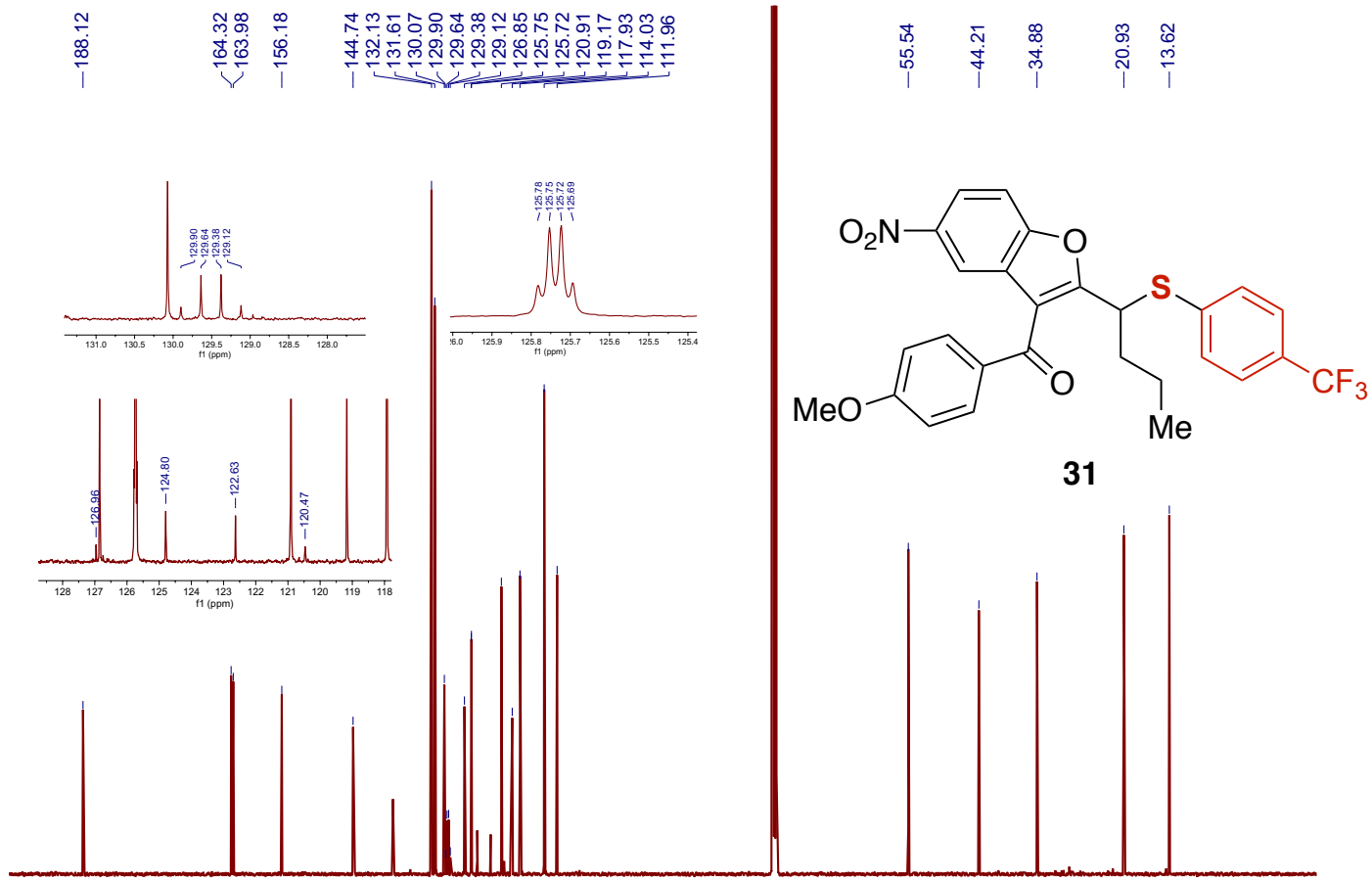

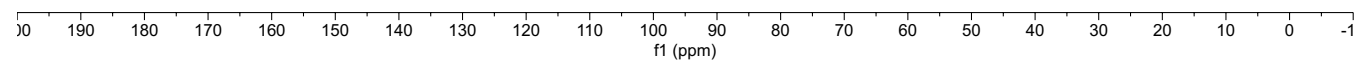


${ }^{19}$ F NMR $\left(377 \mathrm{MHz}, \mathrm{CDCl}_{3}\right)$ of $\mathbf{3 1}$

ஸิ

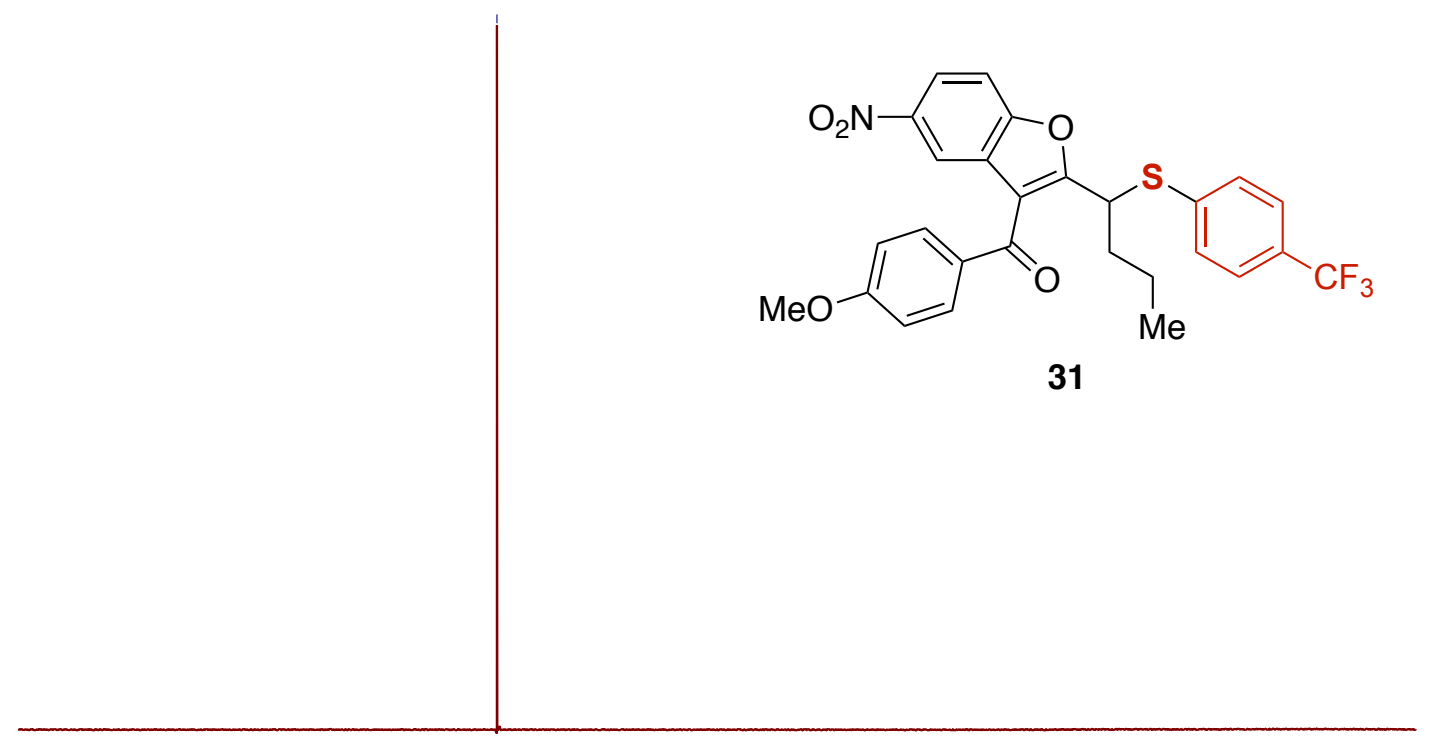

$\begin{array}{llllllllllllllllllllllllll}1 & 10 & 0 & -10 & -20 & -30 & -40 & -50 & -60 & -70 & -80 & -90 & -100 & -110 & -120 & -130 & -140 & -150 & -160 & -170 & -180 & -190 & -200 & -210 & 1\end{array}$ 
${ }^{1} \mathbf{H}$ NMR $\left(500 \mathrm{MHz}, \mathrm{CDCl}_{3}\right)$ of $\mathbf{3 2}$

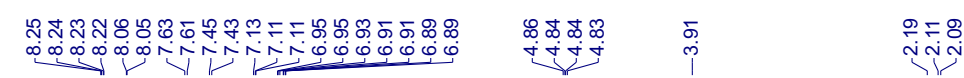

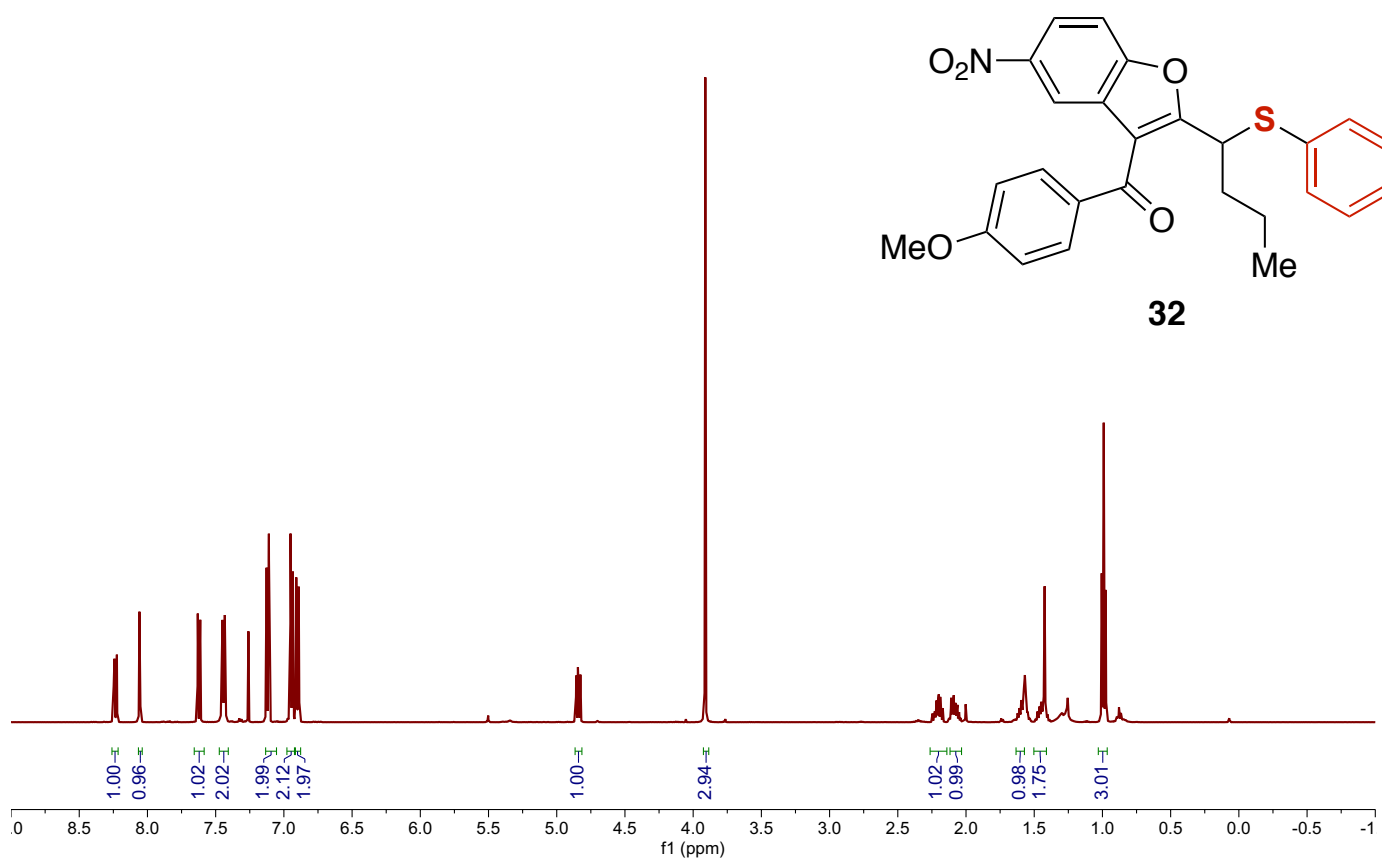

${ }^{13} \mathrm{C}$ NMR $\left(126 \mathrm{MHz}, \mathrm{CDCl}_{3}\right)$ of $\mathbf{3 2}$

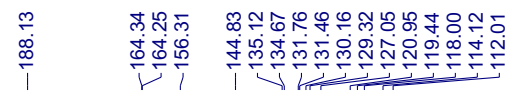

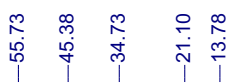

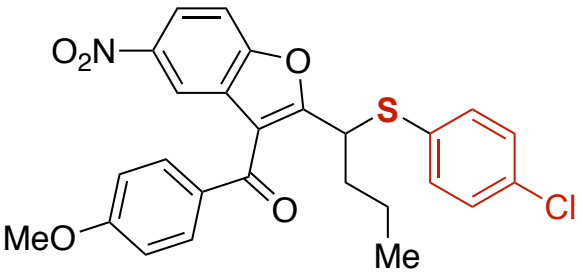

32

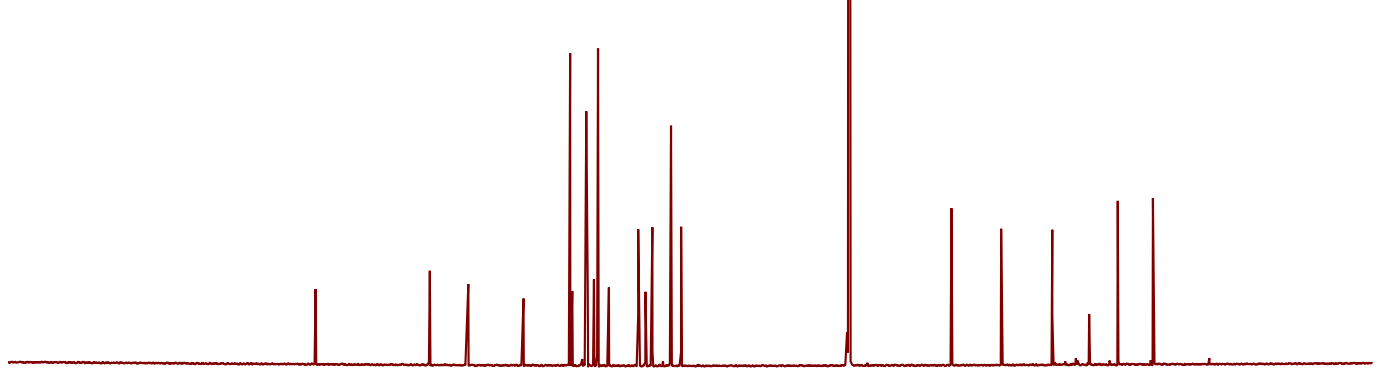

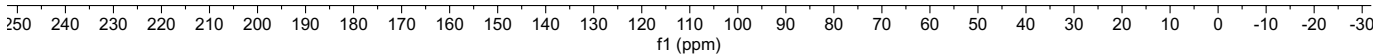


${ }^{\mathbf{1}} \mathbf{H}$ NMR $\left(500 \mathrm{MHz}, \mathrm{CDCl}_{3}\right)$ of $\mathbf{3 3}$

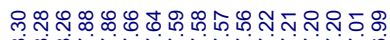

nojivinitivinitivio

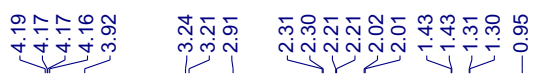

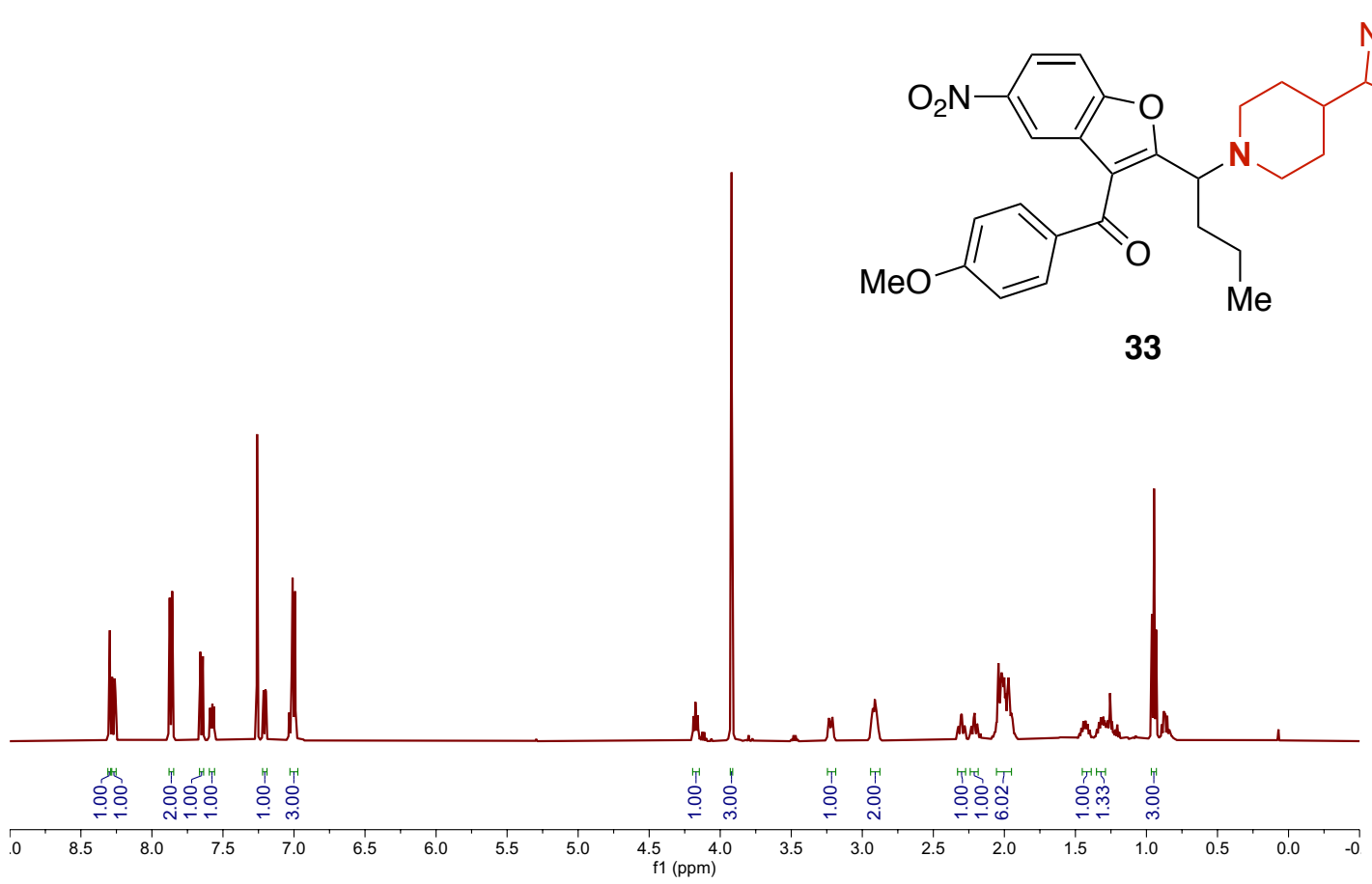

${ }^{13} \mathrm{C}$ NMR $\left(126 \mathrm{MHz}, \mathrm{CDCl}_{3}\right)$ of $\mathbf{3 3}$

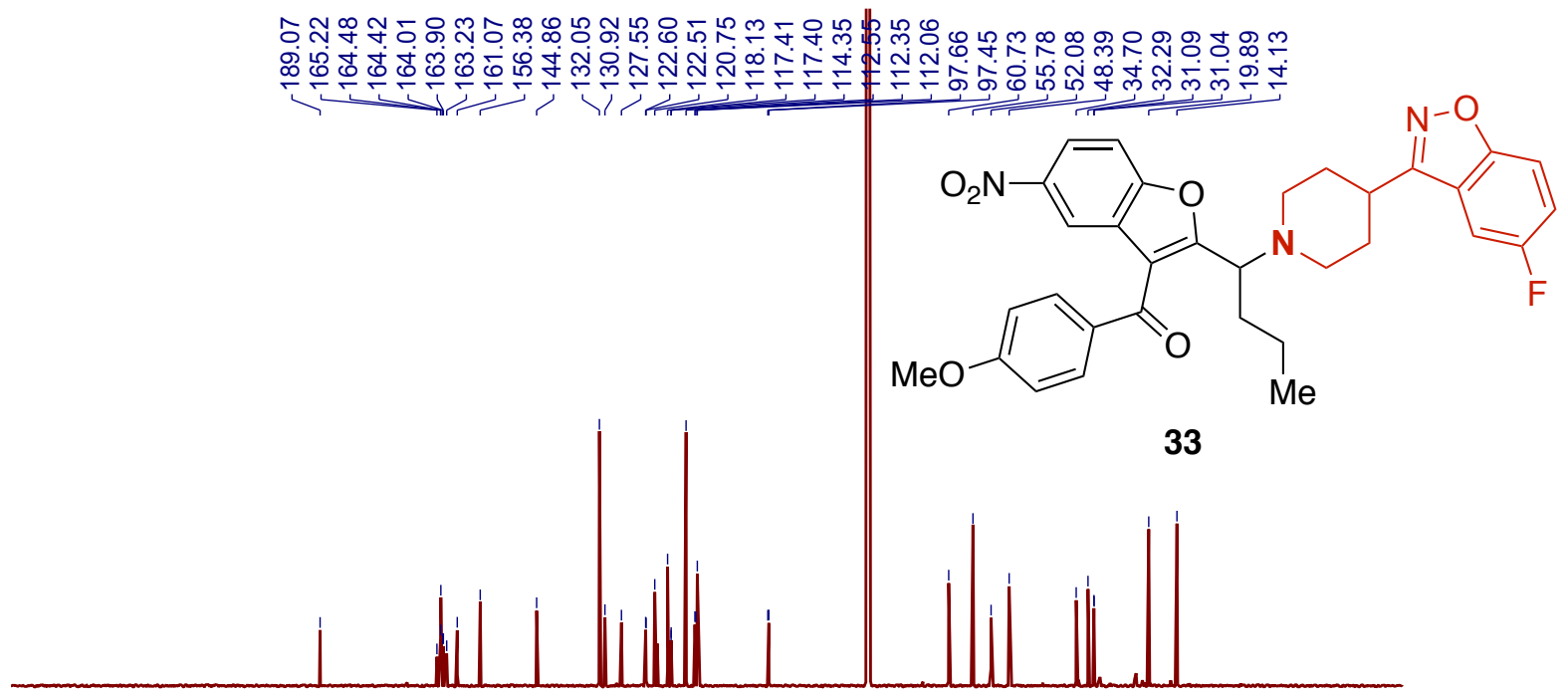

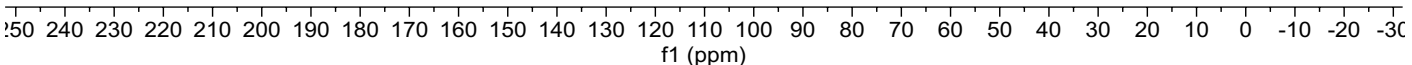


${ }^{19}$ F NMR $\left(377 \mathrm{MHz}, \mathrm{CDCl}_{3}\right)$ of $\mathbf{3 3}$

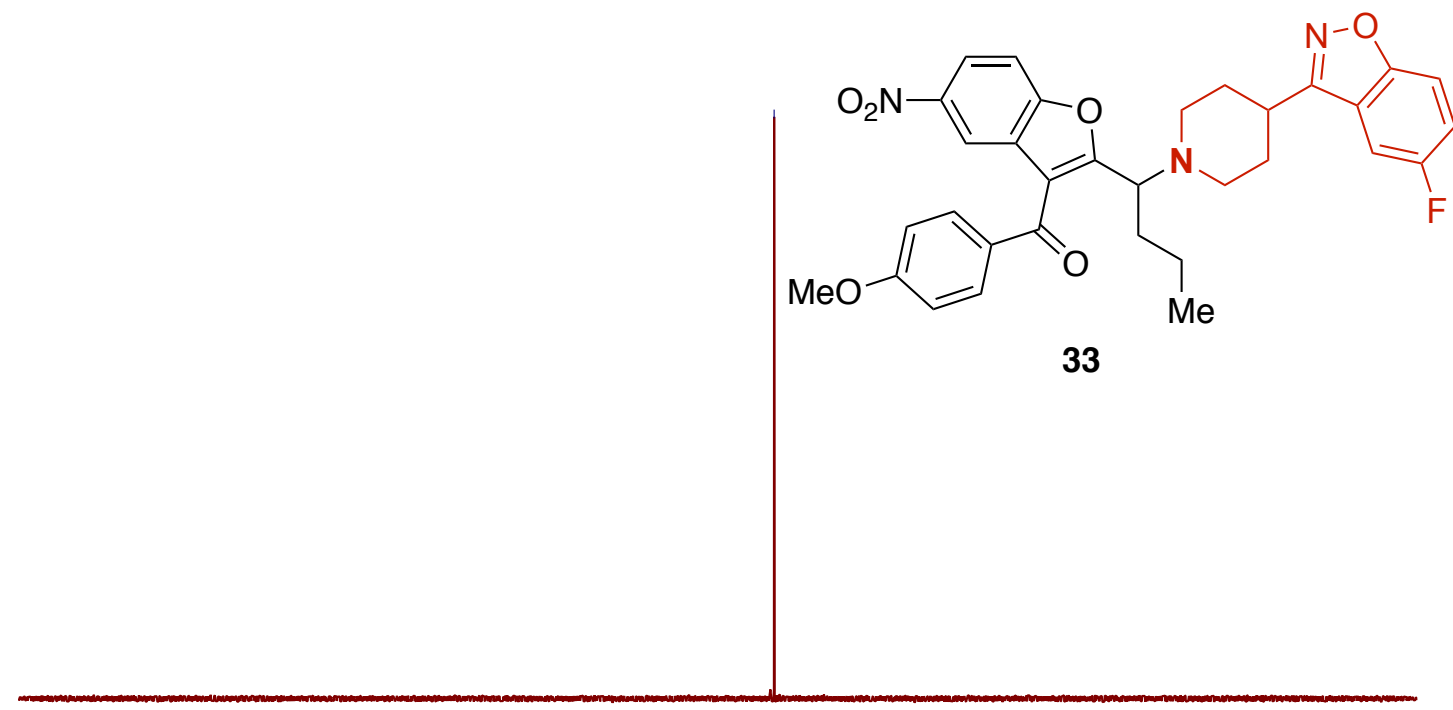

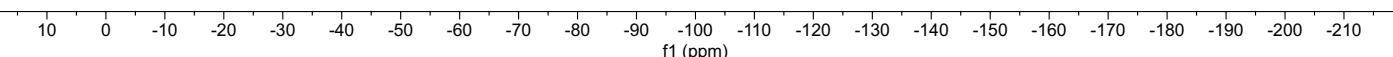


${ }^{1} \mathbf{H}$ NMR $\left(500 \mathrm{MHz}, \mathrm{CDCl}_{3}\right)$ of $\mathbf{3 4}$

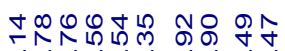

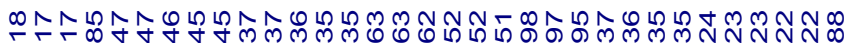
करNî ن

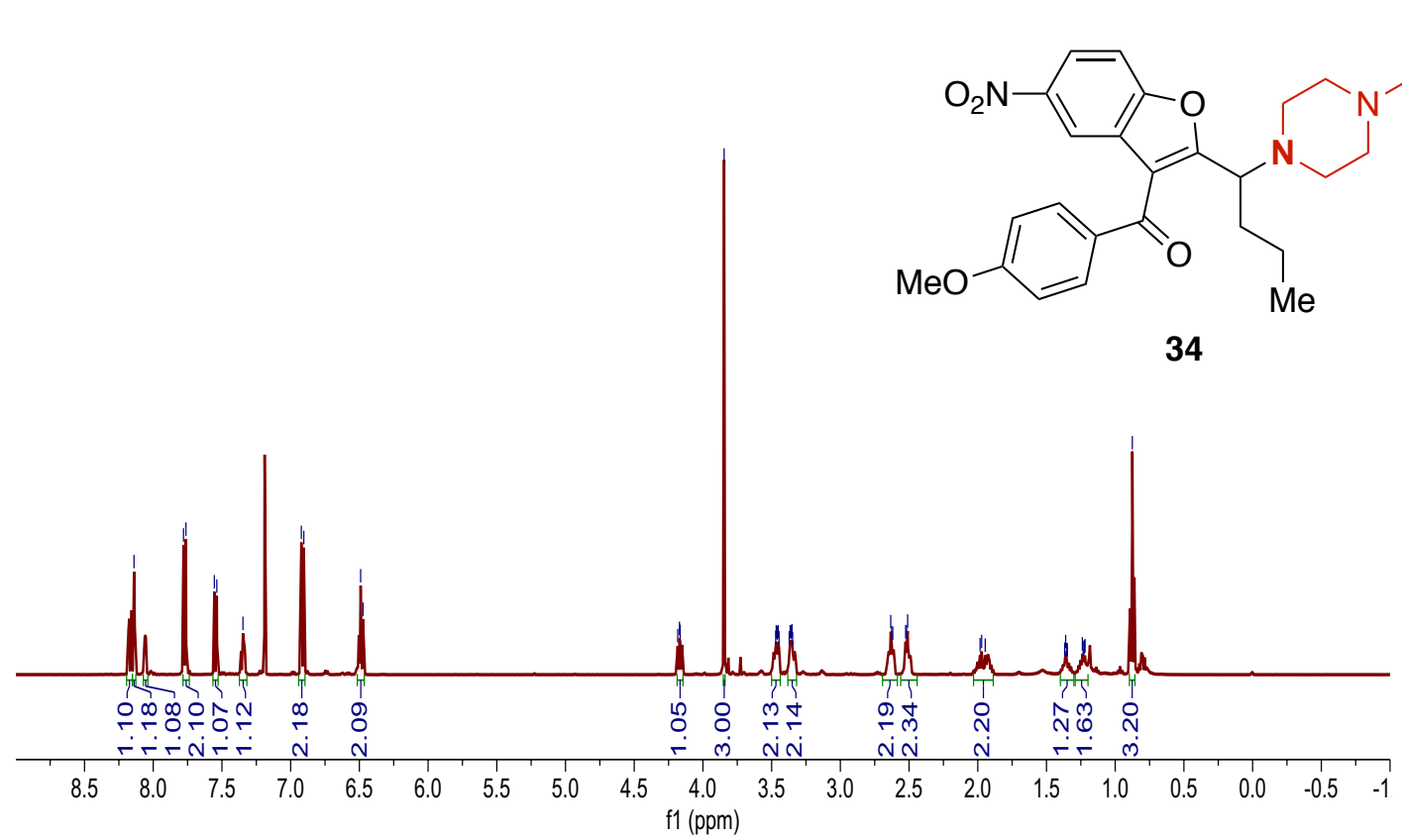

${ }^{13} \mathbf{C ~ N M R}\left(126 \mathrm{MHz}, \mathrm{CDCl}_{3}\right)$ of $\mathbf{3 4}$
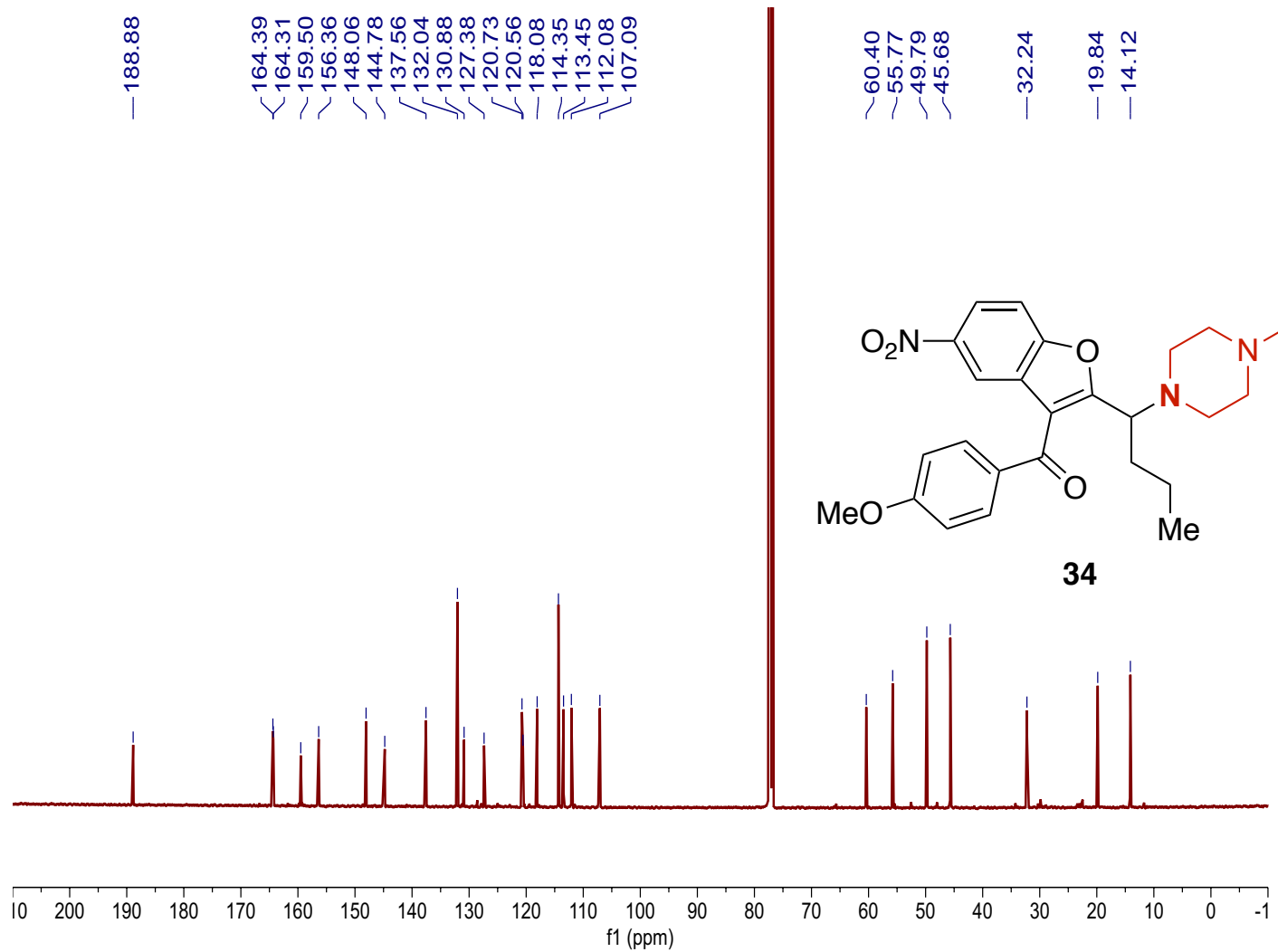